\title{
Interaction of $\mathrm{Ca}^{2+}$ with fully stochastic $\operatorname{InsP}_{3}$ receptor dynamics
}

\author{
Dissertation \\ zur Erlangung des akademischen Grades \\ doctor rerum naturalium \\ (Dr. rer. nat.) \\ im Fach: Physik \\ Spezialisierung: Theoretische Physik \\ eingereicht an der \\ Mathematisch-Naturwissenschaftlichen Fakultät \\ der Humboldt-Universität zu Berlin \\ von \\ Martin Rückl
}

Präsidentin der Humboldt-Universität zu Berlin

Prof. Dr. Sabine Kunst

Dekan der Mathematisch-Naturwissenschaftlichen Fakultät

Prof. Dr. Elmar Kulke
Gutachter/innen:
1. Prof. Benjamin Lindner
2. Prof. Martin Falcke
3. Prof. Barbara Ehrlich

Tag der mündlichen Prüfung: 14.09.2017 


\begin{abstract}
The dynamics of intracellular calcium represents one of the most important signal pathways in cell biology. The emerging calcium patterns control a variety of different functions, including gene expression, cell death, or signal transduction of neurons. Within this work, the focus lies on the hierarchical structure of calcium release events emerging from the feedback of inositol 1,4,5-trisphosphat $\left(\mathrm{IP}_{3}\right)$ receptor $\mathrm{Ca}^{2+}$ ion channel with $\mathrm{Ca}^{2+}$ itself. The topmost level of this hierarchy consists of cell spanning calcium waves or global oscillations involving many clusters of $\mathrm{IP}_{3}$ receptor $\left(\mathrm{IP}_{3} \mathrm{R}\right)$ channels. Release events from individual clusters of channels, called puffs or sparks, constitute the intermediate level. On the finest level, individual channel release events are called blibs.

Within the $\mathrm{Ca}^{2+}$ and $\mathrm{IP}_{3}$ signaling pathway, this work provides insights into the coupling between clusters of $\mathrm{IP}_{3} \mathrm{R}$ and the resulting emergence and termination of waves. To achieve this goal, a stochastic $\mathrm{IP}_{3} \mathrm{R}$ model with non-equilibrium $\mathrm{IP}_{3}$ binding and discrete individual channel states is coupled to two different deterministic descriptions of the released calcium.

The first deterministic description is depicted by a full fledged system of three dimensional reaction diffusion equations of calcium and calcium buffers, which gets solved numerically in a domain around a single cluster. These simulations show that a cluster can produce a variety of non stereotype different puffs, where duration and amplitude distributions of puffs are modulated by the $\mathrm{IP}_{3}$ concentration. For increasing $\mathrm{IP}_{3}$ stimulation, the likelihood to observe a massive, long lasting release event significantly increases, and these events are consequently identified as the source of wave formation. The simulated data is compared to experimental results from Xenopus oocytes, where a similar interspersion of puffs between waves can be observed. Specifically, experiments and simulations show a matching wave period, refractoriness, and puff frequency distribution between wave events, supporting the hypothesis of wave-like events already on a single cluster scale.

The insights of the above high detail model are consequently used to develop a second deterministic description: a coarse grained and phenomenological model based on ordinary differential equations (ODEs). This model allows for sampling of long trajectories of a large system of coupled clusters with reasonable computational effort. Application to a single cluster yields further evidence for the conclusions drawn in the first part. Further, within a grid of coupled clusters, it is showed that the wave-like release events synchronize. While the wave frequency increases with $\mathrm{IP}_{3}$, there is an optimal synchronization for intermediate $\mathrm{IP}_{3}$ excitation. This model also clearly indicates that wave termination is achieved by unbinding of $\mathrm{IP}_{3}$ from the receptor, which essentially renders the channel unable to open, and hence prohibits any further sustained release.
\end{abstract}




\section{Zusammenfassung}

Intrazelluläre Calcium Signale bilden einen der wichtigsten Bestandteile vieler Signalwege in der Zellbiologie. Verschiedene Freisezungsmuster kontrollieren unter anderem die Genexpression, den Zelltod sowie die Signalweiterleitung zwischen Neuronen. Der Fokus dieser Arbeit liegt auf der hierachischen Struktur der Calcium Muster, welche durch die Rückkopplung von inositol 1,4,5-trisphosphat $\left(\mathrm{IP}_{3}\right)$ Rezeptor $\left(\mathrm{IP}_{3} \mathrm{R}\right)$ (Calcium Ionen Kanälen) mit Calcium verursacht wird. Auf der obersten Stufe der Hierarchie stehen zellweite Wellen aus Calcium, welche sich über viele $\mathrm{IP}_{3} \mathrm{R}$ Cluster hinweg erstrecken. Das zweite Level der Hierarchie bilden sogenante Puffs oder Sparks, es entspricht der Freisetzung von Calcium von einzelnen Clustern. Das untere Ende der Hierachie wird durch Blibs gebildet: kleine Calcium Signale verursacht durch einen einzelnen Kanal.

Unter Verwendung zweier verschiedener deterministischer Modelle für die Ausbreitung von Calcium beschäftigt sich diese Arbeit mit der Kopplung zwischen Clustern und der daraus resultierenden Entstehung der Calcium-Wellen innerhalb der oben beschriebenen Hierarchie. Die deterministischen Modelle werden dazu an ein stochastisches Modell mit expliziter Berücksichtigung des $\mathrm{IP}_{3}$ Bindungszustands für individuelle $\mathrm{IP}_{3}$ Kanäle gekoppelt.

Das erste deterministische Modell wird duch ein vollwertiges System dreidimensionaler Reaktions-Diffusions-Gleichungen für Calcium und Calcium-Puffer gebildet. Dieses wird innerhalb einer Clusterumgebung numerisch gelößt. Diese Berechnungen zeigen, dass die Puffs eines Clusters keine stereotypen Ereignisse sind. Sowohl die Dauer, als auch Amplitude der Ereignisse wird durch die $\mathrm{IP}_{3}$ Konzentration geregelt, und die Wahrscheinlichkeit für ein großes, lang andauerndes Ereigniss nimmt mit steigendem $\mathrm{IP}_{3}$ stetig zu. Diese großen Ereignisse werden im Folgenden als die Quelle der Calcium-Wellen indentifiziert. Die simulierten Daten werden mit experimentellen Ergebnissen aus Eizellen der Gattung Xenopus verglichen, bei welchen die Intervalle zwischen Wellen ebenfalls mit einigen Puffs durchsetzt sein können. Insbesondere zeigen die Simulationen zu den Experimenten passende Wellen-Frequenz, Refraktärverhalten und Puff-Frequenz-Verteilung zwischen einzelnen Wellen. Diese Ergebnisse führen zu der Hypothese, dass bereits ein einzelner Cluster ein Calcium Freisetzungsmuster beherrscht, welches im Falle mehrerer Cluster zur Wellenformation führt.

Die Erkenntnisse aus dem Reaktions-Diffusions Modell werden desweiteren für die Entwicklung des zweiten deterministischen Modells verwendet: ein System aus Differentialgleichungen welches mittels mehrerer grober Abschätzungen die Phänomenologie des vorangegangenen Modells abbildet. Dank seiner Einfachheit erlaubt dieses zweite Modell das Sampling von langen Trajektorien für große Systeme mit vergleichbar niedrigem Rechenaufwand. Die daraus resultierende verbesserte Statistik für einzelne Cluster unterstützt die im ersten Teil aufgestellte These. Desweiteren wird anhand eines Systems aus mehreren Clustern gezeigt, wie die wellenartigen Ereignisse einzelner Cluster synchronisieren und die resultierende Frequenz globaler Oszillationen mit steigender Anregung durch $\mathrm{IP}_{3}$ stetig zunimmt. Dabei zeigt die Synchronizität zwischen den Clustern ein lokales Resonanz-Maximum. Zudem wird hier deutlich, dass die Terminierung der Wellen bei dem angewendeten Kanalmodell durch die Dissoziation von $\mathrm{IP}_{3}$ von $\mathrm{IP}_{3} \mathrm{R}$ verursacht wird: bei fehlendem aktivierendem $\mathrm{IP}_{3}$ können die Kanäle die beständige Freisetzung von Calcium nicht aufrecht erhalten und die globale Oszillation oder Welle bricht ab. 
Thanks go to all my fellow PhD students of our work group and especially to my adviser Sten Rüdiger. Further, I want to thank all the people who helped me writing this thesis by providing advice and motivation, especially my parents and my sister! 
I am still confused - but on a higher level.

Enrico Fermi 


\section{Table of Contents}

$\begin{array}{ll}\text { Acronyms } & \text { ix }\end{array}$

Math Symbols $\quad$ xiii

$\begin{array}{ll}\text { Biologic abbreviations } & \text { xv }\end{array}$

1 Introduction 1

2 Theory 5

2.1 Markov Processes . . . . . . . . . . . . . . . . . 5

2.2 Generalized Gillespie Algorithm . . . . . . . . . . . . . . . . 8

2.3 Langevin equation . . . . . . . . . . . . . . . . . . . . 10

2.4 Composed or hybrid Master Equation . . . . . . . . . . . . . 11

2.5 Discretization of continuous space and time . . . . . . . . . . 15

2.5.1 Spatial discretization: Finite Element Method . . . . . . . . . 16

2.5.2 Temporal discretization: 3-stage linearly implicit Runge-Kutta . 18

3 Biology and Models $\quad 21$

3.1 Calcium signaling: the system of interest . . . . . . . . . . 23

3.2 The zoo of calcium patterns . . . . . . . . . . . . . 25

3.3 Modeling . . . . . . . . . . . . . . . . . . . . . 27

3.3.1 Channel gating models . . . . . . . . . . . . . . . 28

3.3.2 Transport and chemical reactions . . . . . . . . . . . . . . 29

3.4 DeYoung-Keizer model for IP3R channel gating . . . . . . . . . . . . . . 31

4 Methods $\quad 35$

4.1 Hybrid schema . . . . . . . . . . . . . . . . . . . 35

4.2 Local spatial refinement and adaptive step size . . . . . . . . . . 38

4.3 Event definitions . . . . . . . . . . . . . . . . . . . . . . . . . . 41

4.4 Synchronization . . . . . . . . . . . . . . . . . . . . 42 
4.4 .1 Event Synchronization . . . . . . . . . . . . . . . . . 42

4.4 .2 Sample coherence function . . . . . . . . . . . . . . . 43

5 Calcium as a concentration field $\quad 47$

5.1 Model description . . . . . . . . . . . . . . . . . . . . . . 47

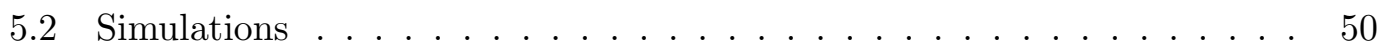

5.3 Results . . . . . . . . . . . . . . . . . . . 52

5.3 .1 Domain size . . . . . . . . . . . . . . . . 52

5.3.2 Micro- and Nanodomain concentration profiles . . . . . . . . 56

5.3 .3 Event definition and lifetime . . . . . . . . . . . . 57

5.3.4 Event lifetime correlates with IP3 binding . . . . . . . . . . . 58

5.3.5 Inhibiton failure and loss of IP3 . . . . . . . . . . . . . . 62

5.3.6 Classification of collective events in puffs and waves . . . . . . 62

5.3.7 Inter wave dynamics and comparison with experimental data . . 65

5.4 Discussion . . . . . . . . . . . . . . . . . 67

6 Coarse grained multi cluster model $\quad 69$

6.1 Model description . . . . . . . . . . . . . . . . . . . 73

6.2 Results . . . . . . . . . . . . . . . . . . . . . 77

6.2 .1 Event definition . . . . . . . . . . . . . . . . . 77

6.2 .2 Single cluster simulations . . . . . . . . . . . . . . 78

6.2 .3 Grid of $8 \times 8$ clusters . . . . . . . . . . . . . . . . . 81

6.2.4 Phaselag distributions between pairs of clusters . . . . . . . . 87

6.3 Discussion . . . . . . . . . . . . . . . . . . . . 89

$\begin{array}{lll}7 & \text { Discussion } & 91\end{array}$ 


\section{Acronyms}

AECR array-enhanced coherence resonance.

AI artificial intelligence.

ATP adenosine triphosphate.

BAPTA 1,2-bis(o-aminophenoxy)ethane-N,N,N',N'-tetraacetic acid.

BC boundary condition.

$\left[\mathrm{Ca}^{2+}\right]$ calcium concentration.

CE collective event.

CCF complex coherence function.

SCF sample coherence function.

CGM coarse grained model.

CICR calcium induced calcium release.

$\left[\mathrm{IP}_{3}\right] \mathrm{IP}_{3}$ concentration.

CTMC continuous-time Markov chain.

CV coefficient of variation.

ODE ordinary differential equation.

PDE partial differential equation.

SDE stochastic differential equation. 
CDF cumulative distribution function.

PDF probability density function.

DUNE distributed and unified numerics environment.

DYK DeYoung-Keizer.

EGTA Ethylene glycol tetraacetic acid.

ER endoplasmic reticulum.

ES event synchronization.

FEM finite element method.

FWHM full width at half maximum.

HDM high detail model.

HEK human embryonic kidney.

IEI inter event interval.

$\mathbf{I P}_{3}$ inositol 1,4,5-trisphosphat.

$\mathbf{I P}_{3} \mathbf{R} \mathrm{IP}_{3}$ receptor.

IPI inter puff interval.

IWI inter wave interval.

MC Markov chain.

MCMC Markov chain Monte Carlo.

MD molecular dynamics.

ML machine learning.

MSC magnitude squared coherence.

PMCA plasma membrane calcium ATPase. 
RyR Ryanodine receptor.

SD standard deviation.

CSD cross spectral density.

PSD power spectral density.

SERCA sarcoplasmic/endoplasmic reticulum calcium ATPase.

SR sarcoplasmic reticulum. 


\section{Math Symbols}

[o] Angular brackets are used for concentrations of chemicals $\circ$.

$B$ The total buffer concentration, possibly with index denoting a specific buffer species.

$b$ The bound buffer concentration, possibly with index denoting a specific buffer species.

$c$ The calcium concentration, possibly depending on space and time.

$D$ The diffusion coefficient of some reactant.

$\Omega$ Some spatial domain.

$\partial \Omega$ The boundary/surface of some domain.

$k^{-}$The unbinding rate of some buffer, sometimes also off-rate, 1 (time $\times$ concentration).

$k^{+}$The binding coefficient of some buffer, sometimes also on-rate, 1/time.

$N$ The number of channels of a cluster.

$n_{\mathrm{o}, i}$ The open fraction of a cluster $i$. 


\section{Biologic abbreviations}

ATP Adenosine triphosphate. A molecule which is used as chemical energy source in various cellular processes.

BAPTA 1,2-bis(o-aminophenoxy)ethane-N,N,N',N'-tetraacetic acid. A rapid high affinity calcium buffer.

$\mathbf{C a}^{2+}$ calcium ion.

CICR Calcium induced calcium release. The process of calcium release from internal stores due to a positive feedback mechanism of ion channels on calcium.

DYK DeYoung-Keizer. A wide used model for $\mathrm{IP}_{3} \mathrm{R}$ channel gating.

EGTA Ethylene glycol tetraacetic acid. A slow high affinity calcium buffer.

ER Endoplasmic reticulum. A organelle which is separated from the cytosol via a lipid bilayer. Contains a high $\left[\mathrm{Ca}^{2+}\right]$.

IP $_{3}$ Inositol 1,4,5-trisphosphat. A messenger protein, designed to regulate $\mathrm{IP}_{3} \mathrm{R} \mathrm{Ca}{ }^{2+}$ ion channels.

$\mathbf{I P}_{3} \mathbf{R} \mathrm{IP}_{3}$ receptor. A $\mathrm{Ca}^{2+}$ ion channel ussually found at ER or SR membranes.

PMCA Plasma membrane calcium ATPase. Pumps $\mathrm{Ca}^{2+}$ from the cytosol through the plasma membrane.

RyR Ryanodine receptor. A calcium ion channel closely related to $\mathrm{IP}_{3} \mathrm{R}$, but regulated by Ryanodine..

SERCA Sarcoplasmic/endoplasmic reticulum calcium ATPase. A $\mathrm{Ca}^{2+}$ pump that pumps $\mathrm{Ca}^{2+}$ from the cytosol to the ER or SR. 
SR Sarcoplasmic reticulum. A organelle similar to the ER. Its main purpose is $\mathrm{Ca}^{2+}$ storage and release. 


\section{Chapter 1}

\section{Introduction}

Machine learning (ML) and artificial intelligence (AI) research will presumably have the largest impact on daily life in first world countries among all fields of science for the upcoming decade. The improvements in both software and hardware tools of the last years resulted in a huge leap forward which already lead to self driving cars and semi autonomous robots. Today, ML allows us to automagically detect cats in online videos [Le+11], and recently the first AIs have beaten professional human players in games like poker $[\mathrm{Bow}+15]$ and Go $[\mathrm{Sil}+16]$.

Besides these fulminant achievements, AI research inherently deals with the quest to understand the emergence of intelligence out of very complex behavior described by a set of relatively simple rules. In biology, these rules correspond to the microscopic physiological processes which drive our brain, and which are ultimately engraved in the human DNA. This explains why the idea of a "whole brain emulation" is discussed as a hot candidate to yield an AI with human cognitive abilities [Bos14]. Of course, understanding the microscopic level of physiology of neurons does not necessarily explain the emergence of intelligence, but it poses a prerequisite for the development of anything close to a "whole brain emulation". Further, because atomistic simulations of a full brain will very likely stay out of reach for the next decades (or even centuries) a concise modeling of the deterministic and stochastic processes on the level of individual neurons (compared to individual atoms or molecules) is necessary to treat such a large scale system on hardware which is, or will be available soon.

Another prominent argument to investigate the microscopical physiology of cells essentially boils down to improving quality and efficiency of healthcare. Understanding the cause of a disease usually requires a comprehensive understanding of the physiological processes involved. If the physiological mechanisms behind the disease are well understood, the process of searching for a treatment changes from meandering exper- 
iments, to directed well-founded research. Therefore, a microscopic understanding of the physiology, with all its possible nonlinearities, also means that a multiplicity of side effects of treatments can be foreseen. In Utopia, this would result in perfect treatments with no side effects.

Biological processes which operate simple unicellular organisms are already complex on their own. Unsurprisingly, for multi-cellular organisms this is even more so, since the organism somehow needs to orchestrate the operation of all its cells. The goal of mathematical physiology in general is to provide models which describe this orchestra. Focusing on the intracellular level specifically, the ultimate goal is a better "microscopic description" of the processes between the vast amount of different protagonists present. Here, the term "microscopic description" intentionally associates with the field of statistical mechanics, since the proximity to the atomistic scale of the intracellular processes often requires probabilistic descriptions of the system evolution. Further, it would be desirable to draw the connection between micro and macro states of a cell, similar as in statistical mechanics.

"Essentially, all models are wrong, but some are useful."[BD87]

The philosophically intriguing questions are: Can one find models which prove useful for the tasks above? Which level of detail is required for the emergence of intelligence and is this level of detail computationally tractable? Which physiological aspects are relevant for the development of a treatment for Alzheimer's disease? As one can see from the above thoughts, a models usefulness depends on the task at hand. But what exactly defines its usefulness? How does one order models according to their usefulness? The usefulness of a model results from a combination of other "meta attributes" like computational complexity, predictive quality, level of detail, number of parameters, or comprehensibility. Depending on the task, some of these attributes are more important than others, and usually there are tradeoffs between them. E.g. higher predictive quality often requires more complex and less comprehensive models.

A very good example for these tradeoffs is the field of theoretical neuroscience, where a large variety of different neuron models compete. For systems of only a single neuron (or a small amount of coupled neurons), detailed neuron models like the Hodgkin Huxley model [HH52], or models with even richer dynamics (e.g. [For15]) are deployed. However this approach is not feasible if the number of neurons grows, and for large networks consisting of thousands or hundreds of thousands of neurons, one uses simplified models like the FitzHugh-Nagumo model [Fit61; NAY62] or "leaky integrate and fire" neurons to study the collective dynamics (see e.g. [Sch+15]).

As in theoretical neuroscience, this work aspires to use best of both sides, in 
the context of intracellular calcium dynamics and its interaction with inositol 1,4,5trisphosphat $\left(\mathrm{IP}_{3}\right)$. Besides its relevance for neuroscience, calcium constitutes to a vast amount of different other cell functions, including gene regulation and cell death. Because of steep concentration gradients between compartments of cells, which are permeable for calcium only via specialized ion channels such as the $\mathrm{IP}_{3}$ receptor $\left(\mathrm{IP}_{3} \mathrm{R}\right)$, a variety of different calcium patterns emerge. There exist traveling waves living almost on the minute time scale in Xenopus oocytes, or cell wide slow global oscillations of the intracellular calcium concentration. On a finer level it becomes clear that these large scale events are constituted by clusters of $\mathrm{IP}_{3} \mathrm{R}$, where each cluster can also produce individual orchestrated release events which are called puffs or sparks. Zooming in even further, individual channels may open or close independently from other channels of their cluster. These atomic release events are called blibs ${ }^{1}$.

Within the $\mathrm{Ca}^{2+}$ and $\mathrm{IP}_{3}$ signaling pathway, this work thrives to provide insights into the coupling between clusters of $\mathrm{IP}_{3} \mathrm{R}$ and the resulting emergence and termination of waves. Therefore, a first high detail model (HDM) will be introduced in chapter 5 . This model has the advantage that it is relatively easy to tune to experiments, since many of its parameters directly resemble controlled environment conditions. The downsides are: a high dimensional parameter space, a very high computational complexity and a lack of intuitive accessibility. Why is a large parameter space a downside? Because the mapping between experiment and model becomes difficult or may have

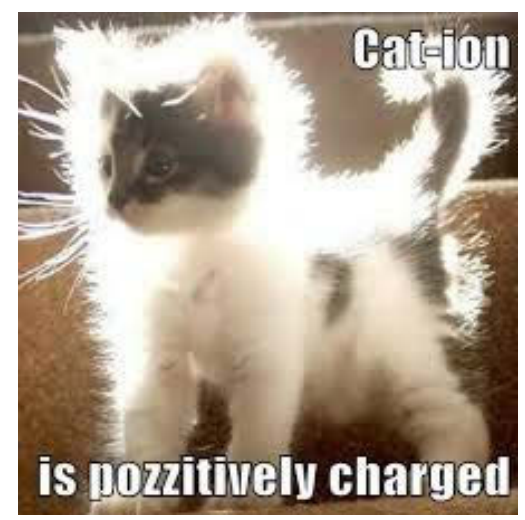

Fig. 1.1: $\mathrm{Ca}^{2+}$ ion detected from an internet video: I haz two charges. multiple solutions, meaning that one experimental observation might be reproducible with two completely different sets of parameters, or vice versa. This model will be used to investigate the release patterns of a single isolated $\mathrm{IP}_{3} \mathrm{R}$.

"With four parameters I can fit an elephant, and with five I can make him wiggle his trunk." John von Neumann

Following the chronological course of research, in chapter 6 a second much more simple coarse grained model (CGM) will be introduced. While one might argue that increasing the level of detail may be more natural, it turns out that the HDM provides a good starting point to understand some of the deterministic properties of $\mathrm{Ca}^{2+}$ release of an individual cluster, and hence provides a good basis for coarse graining. The

\footnotetext{
${ }^{1}$ For a more complete overview about the hierarchy of calcium patterns see section 3.2
} 
CGM in turn allows an effective investigation of stochastic properties: due to its superior computational performance it yields much richer statistics and permits a vastly increased system size. It will be applied to a grid of clusters of $\mathrm{IP}_{3} \mathrm{R}$, and the coupling and synchronization properties between the clusters will be investigated.

The organization of this work is as follows: in the following chapter, the basic theoretical background which is required for the models and their implementation will be introduced. Then, chapter 3 follows with a brief presentation of the relevant parts of $\mathrm{Ca}^{2+}$ signaling and different modeling approaches available. In chapter 4 the focus lies on implementation details of the numerical integration schemata and introduces two different measures for synchronization. The last two chapters will describe, present results, and discuss the HDM and CGM, respectively. While their main constituents are the publications [Ruc+15] and [RR16], they also shed light upon some unpublished relations between the two models. The work concludes with a discussion relating the results of the two models, as well as providing an outlook. 


\section{Chapter 2}

\section{Theory}

The following sections will give a brief introduction into three different topics. The first two sections deal with the concept of Markov processes and the notation of master equations, as they are critical to understanding discrete state ion channel gating models. Then, a very brief introduction into Langevin equations will immediately be followed by what is here called a "hybrid master equation". While it turns out that applying such a hybrid equation to anything but the most simple models is not computationally feasible, the derivation of the hybrid equation gives a deeper insight into the mathematical structure of the interplay between deterministic and probabilistic descriptions of a biological system.

\subsection{Markov Processes}

If one has a stochastic variable $Z(t)$ which depends on time, one speaks of a stochastic process. In general the probability distribution may also depend on time and the values of $Z(t)$ may be correlated for different instants of time. Further, depending on their embedding into time and space, stochastic processes can in general be grouped into four distinct categories:

- Discrete time processes embedded in a discrete state space.

- Discrete time processes embedded in a continuous state space.

- Continuous time processes with discrete state space.

- Continuous time processes with continuous state space. 
For the ease of reading, the following simplified notation will be used: $\rho(z, t)$ denotes the probability ${ }^{1}$ that the stochastic variable $Z$ will have the value $z$ i.e. $\rho(Z=z, t)$ at time $t$.

In the following, the focus lies on a special case of random processes, so called Markov processes, and especially those Markov processes embedded in continuous time. A Markov process is a particularly "simple" stochastic process, because it fulfills the following relation:

$$
\rho\left(z_{n}, t_{n} \mid z_{n-1}, t_{n-1}, z_{n-2}, t_{n-2}, \ldots, z_{1}, t_{1}\right)=\rho\left(z_{n}, t_{n} \mid z_{n-1}, t_{n-1}\right) .
$$

This means that the evolution of the process into another state solely depends on the present state, and not on the history of former states. This relation has some important consequences for other properties of the process. Specifically, it allows to derive the so called Chapman-Kolmogorov equation:

$$
\rho\left(z_{3}, t_{3} \mid z_{1}, t_{1}\right)=\int_{\Omega_{z}} \mathrm{~d} z_{2} \rho\left(\mathrm{z}_{3}, \mathrm{t}_{3} \mid \mathrm{z}_{2}, \mathrm{t}_{2}\right) \rho\left(\mathrm{z}_{2}, \mathrm{t}_{2} \mid \mathrm{z}_{1}, \mathrm{t}_{1}\right) .
$$

Here, the integration covers the full domain $\Omega_{z}$ of possible realizations of $z$, and in case of a discrete state, the integral gets replaced by the respective sum over all possible states. In continuous time, the transition probability in eq. (2.1) can be transformed into transition rates, and one obtains a system of coupled differential equations that describes the evolution of the probability density of the stochastic system:

$$
\frac{\partial}{\partial t} \rho(z, t)=\int \mathrm{dz}^{\prime} \mathrm{w}\left(\mathrm{z}, \mathrm{z}^{\prime}, \mathrm{t}\right) \rho\left(\mathrm{z}^{\prime}, \mathrm{t}\right)-\int \mathrm{dz}^{\prime} \mathrm{w}\left(\mathrm{z}^{\prime}, \mathrm{z}, \mathrm{t}\right) \rho(\mathrm{z}, \mathrm{t}) .
$$

In the above equation, the state space of the process was assumed to be continuous and the master equation is a partial differential equation (PDE). For a discrete state space, the integral transforms into a sum, and the PDE into an ODE:

$$
\frac{\partial}{\partial t} \rho_{n}(t)=\sum_{n^{\prime}}\left[w_{n n^{\prime}}(t) \rho_{n^{\prime}}(t)-w_{n^{\prime} n}(t) \rho_{n}(t)\right]
$$

In the two above equations, the first argument, or index in case of a discrete state, of $w$ denotes the target state, and the second argument the source state. Equation (2.4) can be simplified if one introduces a vector of probabilities $\mathbf{P}=\left(\rho_{1}, \ldots, \rho_{N}\right)$ and a matrix $\widehat{\mathbf{A}}(t)$ holding the transition rates $w_{i j}(t)$ :

$$
\dot{\mathbf{P}}=\widehat{\mathbf{A}}(t) \mathbf{P} .
$$

\footnotetext{
${ }^{1}$ The probability density in case of a continuous process.
} 
Equations (2.3)-(2.5) are called master equations. For a more elaborate discussion of the general equations of Markov processes as well as their derivations, the interested reader is referred to e.g. [Hon02]. Many interesting properties of Markov processes are governed by the properties of the transition matrix A. Here, the focus is on stationarity and detailed balance. For a more complete discussion of other properties (e.g. reversibility, reducibility and periodicity) the interested reader is referred to [Van92].

A Markov process is called homogeneous in time if the transition rates are constant. Then, the solution of eq. (2.5) is straight forward:

$$
\mathbf{P}(t+\tau)=\exp [\tau \mathbf{A}] \mathbf{P}(t) \approx(\mathbf{1}+\tau \mathbf{A}) \mathbf{P}(t) .
$$

In order to conserve the probability over time, the sum over each column of $\mathbf{A}(t)$ needs to vanish: $\sum_{n} A_{n m}(t)=0 \quad \forall \quad t$.

For time homogeneous Markov processes there exists a stationary state $\mathbf{P}^{\infty}$ if the right hand side of eq. (2.5) vanishes, i.e. if there exists a solution $\mathbf{P}^{\infty} \neq 0$ for:

$$
\mathbf{A P}^{\infty}=0
$$

This is equivalent to:

$$
\mathbf{P}^{\infty}=\exp [\tau \mathbf{A}] \mathbf{P}^{\infty} \quad \forall \quad \tau .
$$

Combination of the above equation with eq. (2.4), yields

$$
\sum_{n^{\prime} \neq n} w_{n n^{\prime}} \mathbf{P}_{n^{\prime}}^{\infty}=\sum_{n^{\prime} \neq n} w_{n^{\prime} n} \mathbf{P}_{n}^{\infty} \quad \forall \quad n
$$

as a criterion for stationarity meaning that the influx into each state from any other state has to be balanced with the efflux from this state to any other state. A special case of stationarity is detailed balance. A system fulfills detailed balance if for each pair of states the following relation holds true:

$$
w_{n n^{\prime}} \mathbf{P}_{n^{\prime}}^{\infty}=w_{n^{\prime} n} \mathbf{P}_{n}^{\infty} \quad \forall n \neq n^{\prime} .
$$

This is obviously more restrictive than stationarity, since it does not allow a net flux between any pair of states $n$ and $n^{\prime}$.

Given a stochastic process in the form of eq. (2.1), one can sample sequences of the stochastic variable $Z(t)$, i.e. $\left\{Z\left(t_{0}\right), Z\left(t_{1}\right), \ldots, Z\left(t_{n}\right)\right\}$ where the $t_{i}$ denote the instants of time where the system state undergoes transitions. Such a sequence of states (and their corresponding $t_{i}$ ) is called a Markov chain (MC). The process of sampling such a chain is often referred to as Markov chain Monte Carlo (MCMC), named by the district of the Principality of Monaco and its famous homonymous casino. 
If $\left(x_{0}, \ldots x_{n}\right) \sim \operatorname{MC}\left(\mathbf{P}^{\infty}, \mathbf{A}\right)$ is a Markov chain sampled from the Markov process with stationary state $\mathbf{P}^{\infty}$ and transition matrix $\mathbf{A}$, then $\left(x_{n}, \ldots x_{0}\right)$ is also a Markov chain with the same distribution and transition matrix. This property is called reversibility and Markov processes that satisfy detailed balance are always reversible. Further, if detailed balance is fulfilled, the transition matrix $\mathbf{A}$ is diagonalizable, i.e. there exists an invertible matrix $\mathbf{M}$ such that $\mathbf{M}^{-1} \mathbf{A M}$ is diagonal.

\subsection{Generalized Gillespie Algorithm}

The previous section has given a brief introduction into Markov processes. In order to sample Markov chains for transition matrices $\mathbf{A}(t)$ that depend on time, a proper way to draw samples from the probability density function (PDF) of intervals between two successive jumps is required. This probability distribution is called the waiting time distribution $P_{\mathrm{w}}(\tau)$ and it's complementary distribution is the dwell time distribution or survival function. The dwell time distribution $P_{\mathrm{d}}(\tau)$ defines the probability that no jump occurs in the interval $(t, t+\tau)$.

Defining the total jump rate of a state $S$ as

$$
a_{S}(t)=\sum_{S^{\prime} \neq S} w_{S^{\prime} S}(t)
$$

the dwell time distribution, a cumulative distribution function (CDF), follows as [Hon02]:

$$
P_{\mathrm{d}}(\tau \mid S, t)=\exp \left[-\int_{t}^{t+\tau} \mathrm{dt}^{\prime} \mathrm{as}_{\mathrm{S}}\left(\mathrm{t}^{\prime}\right)\right]
$$

As stated above, the waiting time distribution is just the complementary of $P_{\mathrm{d}}$ :

$$
P_{\mathrm{w}}(\tau)=1-P_{\mathrm{d}}(\tau)
$$

In order to draw a random number from the distribution $P_{\mathrm{w}}(\tau)$, and hence get the duration $\tau$ until the next event occurs one first draws a uniform random number $\chi \in$ $[0,1)$, and then solves the following equation for $\tau$ :

$$
P_{\mathrm{w}}(\tau)=1-P_{\mathrm{d}}(\tau)=1-\exp \left[-\int_{t}^{t+\tau} \mathrm{dt}^{\prime} \mathrm{a}_{\mathrm{S}}\left(\mathrm{t}^{\prime}\right)\right]=\chi .
$$

In general, this requires the numerical solution of:

$$
-\ln [1-\chi]=\int_{t}^{t+\tau} \mathrm{dt}^{\prime} \mathrm{a}_{\mathrm{S}}\left(\mathrm{t}^{\prime}\right)
$$




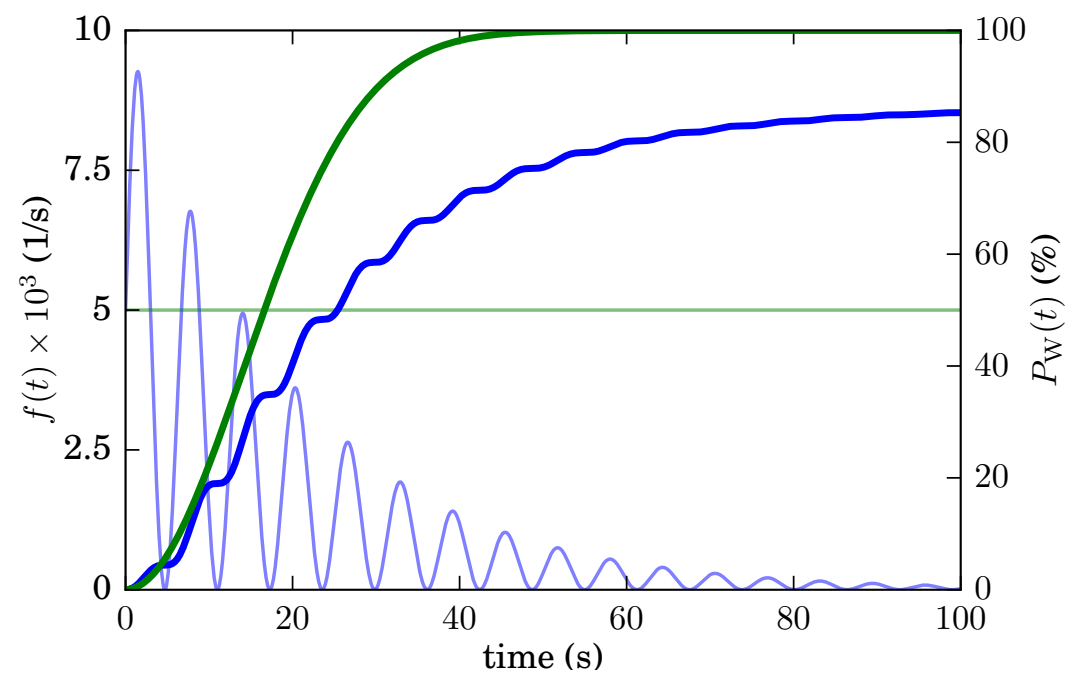

Fig. 2.1: Reaction rates $f(t)$ (left axis) and waiting time distribution $P_{\mathrm{w}}(t)$ (right axis) for a homogeneous (green) and an inhomogeneous (blue) Markov process. As one can see, for the inhomogeneous Markov process, eq. (2.14) has no solution if $\chi>0.9$ due to the rapid decay of the frequency.

However, for a homogeneous Markov process $(\mathbf{A}=$ const. $)$ eq. (2.13) simplifies to:

$$
P_{\mathrm{w}}(\tau)=1-\exp \left[-\tau a_{S}\right]
$$

and one can simply draw random waiting times by transforming the uniform random variable $\chi$ according to:

$$
\tau=-\frac{\ln [1-\chi]}{a_{S}}
$$

This equation for the special case of homogeneous Markov processes was derived by Daniel T. Gillespie in [Gil76] and was applied to chemical reactions. Therefore, the extension to time dependent rates is here called generalized Gillespie algorithm (see e.g. [Gil91]).

One important, difference between the original Gillespie algorithm and the generalized version is, that eq. (2.14) will always have a solution for $\tau$ if the reaction rate is constant. This is not necessarily the case for a time dependent reaction rate $a(t)$, because $P_{\mathrm{w}}(\tau)$ does not always tend to unity for large $\tau$ (see fig. 2.1. The simple interpretation of this is: if the rates $w_{n n^{\prime}}(t)$ vanish, no more transitions will occur.

For the sampling of a Markov chain (MC), the remaining task is to determine the target state to which the system will jump. Because of the Markov property of the 
process, the probability to jump into any other state $S^{\prime}$ at time $t+\tau$ does only depend on the rates $w_{S^{\prime} S}(t+\tau)$ and $a_{S}(t+\tau)$. Hence, it is given by the simple relation:

$$
P\left(S_{\text {target }}=S^{\prime}\right)=\frac{w_{S^{\prime} S}(t+\tau)}{a_{S}(t+\tau)} .
$$

With eqs. (2.15) and (2.18) one can sample trajectories of any Markov process that has a master equation given like eq. (2.5).

\subsection{Langevin equation}

A different approach to treat random fluctuations of many real world dynamical systems is pursued with stochastic differential equations (SDEs). SDEs extend the concept of differential equations by introducing randomness into the mathematical description. In its most general form, a SDE can be written as:

$$
\frac{\partial x_{i}}{\partial t}=f_{i}\left(\mathbf{x}, \xi_{1}(t), \ldots \xi_{m}(t), t\right)
$$

where $\xi_{j}(t)$ are random processes and $\mathbf{x}=\left(x_{1}, \ldots, x_{n}\right)^{T}$ is a set of dynamic variables. In contrast to deterministic differential equations, the evolution of such a system is not predefined by an initial condition. Instead, each integration of a SDE can yield different realizations of trajectories due to the randomness of the noise process. On the other hand, a SDE allows to intermix a deterministic evolution with a random process, which isn't possible in a straight forward way with the master equations described in section 2.1.

If one can split the functions $f_{i}$ of eq. (2.19) into a deterministic and a stochastic part, one yields the most general form of a Langevin equation:

$$
\frac{\partial x_{i}}{\partial t}=f_{i}(\mathbf{x}, t)+g_{i}\left(\mathbf{x}, \xi_{1}(t), \ldots \xi_{m}(t), t\right) .
$$

Two special cases of the Langevin equation are additive and multiplicative noise, respectively:

$$
\begin{aligned}
& \frac{\partial x_{i}}{\partial t}=f_{i}(\mathbf{x}, t)+\sum_{j} g_{i j} \xi_{j}(t) \\
& \frac{\partial x_{i}}{\partial t}=f_{i}(\mathbf{x}, t)+\sum_{j} g_{i j}(\mathbf{x}, t) \xi_{j}(t)
\end{aligned}
$$

where in the case of additive noise $g_{i j}$ are simple constant factors. In contrast, if $g_{i j}$ is a function of any $x_{i}$ the noise is called multiplicative. 
Langevin equations have been applied in various fields with a large variety of different noise forms (see e.g. [Ani+07]). In this work, one type of noise will be of particular interest: dichotomous noise. For dichotomous noise, the stochastic variable can only choose between two different values $I_{t}= \pm \Delta$ :

$$
\frac{\partial x}{\partial t}=f(x)+g(x) I_{t}
$$

The main difference between dichotomous noise and other noise forms is, that the time evolution of a dichotomous process is not given by another Langevin equation (like e.g. in the Ornstein-Uhlenbeck process), but by a master equation like eq. (2.4)! In the following section a more general form of eq. (2.23) will be introduced. Then, a master equation for such a system will be derived and an integration schema which allows to sample trajectories from it will be provided.

\subsection{Composed or hybrid Master Equation}

The dichotomous noise described in the previous section is a very specific (and probably the most simple) form of discrete valued noise. Here, a generalized version of eq. (2.23) is provided and the corresponding master equation is derived.

In a generalized form of eq. (2.23), the variable $x$ may be a $K$ dimensional vector. Further, the discrete state is not restricted to two different realizations, but may take any value from a defined set $\mathcal{S}=\left\{S_{1}, \ldots, S_{N}\right\}$ of values. Equation (2.23) then reads:

$$
\frac{\mathrm{d} x_{k}}{\mathrm{~d} t}=f_{k}(\mathbf{x}, t)+g_{k}(\mathbf{x}, S(t), t) \text { with } S(t) \in \mathcal{S}
$$

where $S(t)$ evolves according to an inhomogeneous master equation

$$
\frac{\mathrm{d} P\left(S(t)=S_{n}\right)}{\mathrm{d} t} \equiv \frac{\mathrm{d} \rho_{n}}{\mathrm{~d} t}=\sum_{n^{\prime}}\left[w_{n n^{\prime}}(\mathbf{x}, t) \rho_{n^{\prime}}(t)-w_{n^{\prime} n}(\mathbf{x}, t) \rho_{n}(t)\right] .
$$

Equation (2.25) poses another difference to a normal dichotomous process, as the rates for switching between states depend on the dynamic variables: $w_{n n^{\prime}}=w_{n n^{\prime}}(\mathbf{x}, t)$.

Similar as for the simple dichotomous noise process, a master equation for eq. (2.24) will describe the evolution of the joint probability to find the system at a specific value x and state $S_{n}$. Hence, the master equation will be a system of $K \times N$ coupled PDEs describing the probability densities of $x_{k}$ for all different states $S_{n}$.

The following equations will closely resemble the derivation of the master equation eq. (2.3) as given in [Hon02] (Chapter 5.2). A similar derivation is given in [Ani+07] (Chapter 1.2). However, due to the coupling of the two systems, some additional 
calculations are required. Further, $p(i, t+\tau \mid j, t)$ will denoted the probability to be in state $S_{i}$ at time $t+\tau$ given state $S_{j}$ at time $t$. For the sake of simplicity and readability, assume that there is only one dynamic variable, i.e. $\mathbf{x} \equiv x$.

As showed in [Hon02], the master equation can be derived from the short term evolution of the probability. Obviously, a transition probability needs to fulfill the following relation:

$$
p(i, t+\tau \mid j, t) \rightarrow \delta_{i j} \text { for } \tau \rightarrow 0
$$

Considering also first order terms of $\tau$ in the expansion leads to the short term expansion of $p$ which reads:

$$
p(i, t+\tau \mid j, t)=\delta_{i j}+\tau\left[w_{i j}(t)-a_{j}(t) \delta_{i j}\right]+O\left(\tau^{2}\right) .
$$

Since probability needs to be conserved, the relation $a_{j}(t)=\sum_{k} w_{k j}(t)$ needs to hold true for all times $t$.

Although the above formalism was derived for a stochastic system, one can just use the same schema to describe the evolution of a deterministic process as well. Given the differential equation $\dot{x}(t)=f(x, t)$ and its solution $x(t)$ for the initial condition $x_{0}$ at $t_{0}$ one can write:

$$
p\left(z, t \mid x_{0}, t_{0}\right)=\delta(z-x(t)) .
$$

The above form ensures that one will always measure the values determined by the differential equation. The short term expansion follows as:

$$
\begin{aligned}
p\left(x, t+\tau \mid x^{\prime}, t\right) & =\delta\left(x-\left(x^{\prime}+\tau f\left(x^{\prime}, t\right)\right)\right) \\
& =\delta\left(x-x^{\prime}\right)-\tau f\left(x^{\prime}, t\right) \partial_{x} \delta\left(x-x^{\prime}\right)+O\left(\tau^{2}\right) \\
& =\delta\left(x-x^{\prime}\right)+\tau \omega\left(x, x^{\prime}, t\right)+O\left(\tau^{2}\right) .
\end{aligned}
$$

Here the factor $\omega\left(x, x^{\prime}, t\right)=f\left(x^{\prime}, t\right) \partial_{x} \delta\left(x-x^{\prime}\right)$ denotes the product which arises from the first order Taylor expansion. ${ }^{2}$

In order to combine the deterministic and the stochastic part, the first step is to think of eq. (2.24) as a system of deterministic equations, one for each distinct stochastic state $S_{n}$. I.e. one replaces

$$
\frac{\mathrm{d} x}{\mathrm{~d} t}=f(x, t)+g(x, S(t), t)
$$

\footnotetext{
${ }^{2}$ In case of a vector of dynamic variables $\mathbf{x}$ this is a scalar product!
} 
with $^{3}$

$$
\frac{\mathrm{d} x_{i}}{\mathrm{~d} t}=f_{i}(x, t) \equiv f(x, t)+\sum_{j} \delta_{i j} g\left(x, S_{j}, t\right) \text { with } i \in\{1, \ldots, N\} .
$$

Then, the short term expansion of the joint probability distribution for the composed state $\{i, x\}$ is given by the product of the two subsystems' short term expansions from eqs. (2.27) and (2.29):

$$
\begin{aligned}
p\left(i, x, t+\tau \mid j, x^{\prime}, t\right)= & p\left(i, x^{\prime}, t+\tau \mid j, x^{\prime}, t\right) p\left(j, x, t+\tau \mid j, x^{\prime}, t\right) \\
= & \delta_{i j} \delta\left(x-x^{\prime}\right)-\tau a_{j}\left(x^{\prime}, t\right) \delta_{i j} \delta\left(x-x^{\prime}\right)+ \\
& \tau w_{i j}\left(x^{\prime}, t\right) \delta\left(x-x^{\prime}\right)+\tau \omega_{j}\left(x, x^{\prime}, t\right) \delta_{i j}+O\left(\tau^{2}\right) .
\end{aligned}
$$

Here it becomes clear that the functions $a_{i}, w_{i j}$ and $\omega_{j}$ can all have an explicit dependence on time. ${ }^{4}$ In the following, these dependencies will be omitted again. Using the Chapman-Kolmogorov eq. (2.2) and eq. (2.32), one can write the conditional short term expansion as:

$$
\begin{aligned}
p\left(i, x, t+\tau \mid j, x^{\prime}, t^{\prime}\right)= & \sum_{k} \int \mathrm{dx}^{\prime \prime} \mathrm{p}\left(\mathrm{i}, \mathrm{x}, \mathrm{t}+\tau \mid \mathrm{k}, \mathrm{x}^{\prime \prime}, \mathrm{t}\right) \mathrm{p}\left(\mathrm{k}, \mathrm{x}^{\prime \prime}, \mathrm{t} \mid \mathrm{j}, \mathrm{x}^{\prime}, \mathrm{t}^{\prime}\right)= \\
= & \sum_{k} \int \mathrm{dx}^{\prime \prime} \delta_{\mathrm{ik}} \delta\left(\mathrm{x}-\mathrm{x}^{\prime \prime}\right) \mathrm{p}\left(\mathrm{k}, \mathrm{x}^{\prime \prime}, \mathrm{t} \mid \mathrm{j}, \mathrm{x}^{\prime}, \mathrm{t}^{\prime}\right) \\
& -\tau \sum_{k} \int \mathrm{dx}^{\prime \prime} \mathrm{a}_{\mathrm{k}}\left(\mathrm{x}^{\prime \prime}\right) \delta_{\mathrm{ik}} \delta\left(\mathrm{x}-\mathrm{x}^{\prime \prime}\right) \mathrm{p}\left(\mathrm{k}, \mathrm{x}^{\prime \prime}, \mathrm{t} \mid \mathrm{j}, \mathrm{x}^{\prime}, \mathrm{t}^{\prime}\right) \\
& +\tau \sum_{k} \int \mathrm{dx}^{\prime \prime} \mathrm{w}_{\mathrm{ik}}\left(\mathrm{x}^{\prime \prime}\right) \delta\left(\mathrm{x}-\mathrm{x}^{\prime \prime}\right) \mathrm{p}\left(\mathrm{k}, \mathrm{x}^{\prime \prime}, \mathrm{t} \mid \mathrm{j}, \mathrm{x}^{\prime}, \mathrm{t}^{\prime}\right) \\
& +\tau \sum_{k} \int \mathrm{dx}^{\prime \prime} \omega_{\mathrm{k}}\left(\mathrm{x}, \mathrm{x}^{\prime \prime}\right) \delta_{\mathrm{ik}} \mathrm{p}\left(\mathrm{k}, \mathrm{x}^{\prime \prime}, \mathrm{t} \mid \mathrm{j}, \mathrm{x}^{\prime}, \mathrm{t}^{\prime}\right) \\
= & p\left(i, x, t \mid j, x^{\prime}, t^{\prime}\right)-\tau a_{i}(x) p\left(i, x, t \mid j, x^{\prime}, t^{\prime}\right) \\
& +\tau \sum_{k} w_{i k}(x) p\left(k, x, t \mid j, x^{\prime}, t^{\prime}\right)+\tau \int \mathrm{dx}{ }^{\prime \prime} \omega_{\mathrm{i}}\left(\mathrm{x}, \mathrm{x}^{\prime \prime}\right) \mathrm{p}\left(\mathrm{i}, \mathrm{x}^{\prime \prime}, \mathrm{t} \mid \mathrm{j}, \mathrm{x}^{\prime}, \mathrm{t}^{\prime}\right) .
\end{aligned}
$$

Using the normalization $a_{i}=\sum_{k} w_{k i}$ and calculating the limit for $\tau \rightarrow 0$ yields the

\footnotetext{
${ }^{3}$ Don't get confused, the vector notation of $x$ got dropped, hence $i$ does denote the index of the state $S$.

${ }^{4}$ Because different functions $f_{j}$ are considered, there also appears an index $j$ at $\omega$.
} 
derivative of the conditional probability w.r.t. time:

$$
\begin{aligned}
\frac{\partial p\left(i, x, t \mid j, x^{\prime}, t^{\prime}\right)}{\partial t}= & \sum_{k}\left[w_{i k}(x) p\left(k, x, t \mid j, x^{\prime}, t^{\prime}\right)-w_{k i}(x) p\left(i, x, t \mid j, x^{\prime}, t^{\prime}\right)\right] \\
& +\int \mathrm{d} \mathrm{x}^{\prime \prime} \omega_{\mathrm{i}}\left(\mathrm{x}, \mathrm{x}^{\prime \prime}\right) \mathrm{p}\left(\mathrm{i}, \mathrm{x}^{\prime \prime}, \mathrm{t} \mid \mathrm{j}, \mathrm{x}^{\prime}, \mathrm{t}^{\prime}\right)
\end{aligned}
$$

Finally, one multiplies with $p\left(j, x^{\prime}, t^{\prime}\right)$, integrates over $x^{\prime}$ and sums over $j$ :

$$
\frac{\partial p(i, x, t)}{\partial t}=\sum_{k}\left[w_{i k}(x) p(k, x, t)-w_{k i}(x) p(i, x, t)\right]+\int \mathrm{dx}^{\prime} \omega_{\mathrm{i}}\left(\mathrm{x}, \mathrm{x}^{\prime}\right) \mathrm{p}\left(\mathrm{i}, \mathrm{x}^{\prime}, \mathrm{t}\right) .
$$

This is the master equation for the coupled system. One can rewrite it as:

$$
\begin{aligned}
\frac{\partial p_{i}(x, t)}{\partial t} & =\sum_{k}\left[w_{i k}(x) p_{k}(x, t)-w_{k i}(x) p_{i}(x, t)\right]+\int \mathrm{dx}^{\prime} \omega_{\mathrm{i}}\left(\mathrm{x}, \mathrm{x}^{\prime}\right) \mathrm{p}_{\mathrm{i}}\left(\mathrm{x}^{\prime}, \mathrm{t}\right) \\
\frac{\partial \mathbf{p}(x, t)}{\partial t} & =\mathbf{A}(x, t) \mathbf{p}(x, t)+\int \mathrm{dx}^{\prime} \boldsymbol{\Omega}\left(\mathrm{x}, \mathrm{x}^{\prime}, \mathrm{t}\right) \mathbf{p}\left(\mathrm{x}^{\prime}, \mathrm{t}\right) .
\end{aligned}
$$

Here, the matrix $\mathbf{A}$ denotes the transition rates between the discrete stochastic states, and the diagonal matrix $\boldsymbol{\Omega}$ encodes the deterministic dynamics. The normalization conditions of such a system read:

$$
\begin{aligned}
& \sum_{j} \int \mathrm{dx} \mathrm{p}_{\mathrm{j}}(\mathrm{x}, \mathrm{t})=1 \quad \forall \quad t \\
& \int \mathrm{dx} \mathrm{p}_{\mathrm{j}}(\mathrm{x}, \mathrm{t} \mid \mathrm{j})=1 \quad \forall \quad t \\
& \sum_{j} p_{j}(x, t \mid x)=1 \quad \forall \quad t .
\end{aligned}
$$

Two other very interesting quantities are the probability to find the system in a specific discrete or continuous state, independent of the respective other state:

$$
\begin{aligned}
P_{j}(t) & =\int \mathrm{dx} \mathrm{p}_{\mathrm{j}}(\mathrm{x}, \mathrm{t}) \\
p(x, t) & =\sum_{k} p_{k}(x, t) .
\end{aligned}
$$

\section{Spatially dependent SDEs}

In the previous section no constraints were put on the dynamic variable $x$. Therefore one might want to assume that $x$ depends not only on time but also is a function of 
spatial coordinates $\mathbf{q} \in \mathcal{R}^{d}$. While the derivation of the above equations in principle is valid for such cases, some things need to be taken care of. This becomes apparent already from eq. (2.29). Here $f$ may contain partial derivatives of $x$ w.r.t. $q_{i}$ which does not make sense if $x^{\prime}$ simply was a random variable. ${ }^{5}$ Instead, one has to think of $x$ as a $d$-dimensional random field. Then, the resulting probability density will also depend on $\mathrm{q}$ and all expressions like $\int \mathrm{d} x^{\prime}$ will turn into infinite dimensional integrals $\left(\int \cdots \int\right)^{d} \mathrm{~d}\left(x^{\prime}(\mathbf{q})\right)^{d}$. Note that for this case the functions $a_{i}, w_{i j}$ and $\omega_{j}$ can also depend on $\mathbf{q}$.

Of course, one could discretize space with a finite subsets of points $\left\{\mathbf{q}_{1} \ldots, \mathbf{q}_{L}\right\}$. Then the random field can be denoted as a random vector and the above integrals turn into something more tractable like $\int \cdots \int \mathrm{d}^{1} x^{\prime} \ldots \mathrm{d}^{L} x^{\prime}$. However, this will result in a system of $L \times K \times N$ coupled master equations, where $K$ and $N$ are the number of dynamic variables and the number of distinct states, respectively.

Obviously, when following this approach, the complexity of the system that has to be solved will explode. In fact, solving even the most simple model consisting of only a single dynamic variable which only depends on time, would require a massive computational effort. The above method is therefore primarily of academic usefulness and its direct application is constrained to very simple systems consisting of only a few distinct states $S_{i}$, a simple model, and a single or very few dynamic variables.

\subsection{Discretization of continuous space and time}

The models introduced later in this work require the integration of systems of coupled PDEs or coupled ODEs. Therefore, this section gives a short overview on the mathematical techniques used for integrating such systems. First, a short introduction into the finite element method (FEM) is provided, which will be used to transform a system of PDEs into a much larger system of ODEs. This large system of ODEs is only sparsely coupled, since single equations describe the solution of the PDE at specific points in space, which only interact with their neighbouring points. Applying the finite element method (FEM) yields a so called semi-discrete describtion of the PDE. The semi discrete system then needs to be integrated leading to a fully discrete system in space and time. For this, a Runge-Kutta base integration technique for coupled ODEs is introduced, which allows for efficient and adaptive time discretization.

\footnotetext{
${ }^{5} \mathrm{Or}$ a random vector if there is more than one dynamic variable.
} 


\subsubsection{Spatial discretization: Finite Element Method}

The goal of this chapter is not to provide a mathematically rigorous description of the FEM, but a general understanding of the steps that lead to the semi discrete description. For a full detailed introduction into the general topic see e.g. [Red14] and for mathematically rigorous descriptions see [DW12]. Further, the PhD thesis of Nagaiah Chamakuri $[\mathrm{Nag}+07]$ provides an excellent overview with direct applications to calcium diffusion equations.

Consider the abstract problem:

$$
\begin{aligned}
\frac{\partial u(\mathbf{x}, t)}{\partial t} & =F(u, \mathbf{x}, t) \quad \text { or } \\
\frac{\partial u(\mathbf{x}, t)}{\partial t}-F(u, \mathbf{x}, t) & =0 \quad \text { with } \quad \mathbf{x} \in \mathbb{R}^{d}
\end{aligned}
$$

where $F(u, \mathbf{x}, t)$ may contain various partial derivatives $\partial_{x_{i}} u$ of $u$ w.r.t. $x_{i}$.

The first step is to discretize the space of interest $\Omega$ into non overlapping finite elements $\Omega_{i}$ such that:

$$
\Omega=\bigcup \Omega_{i} \text { and } \Omega_{i} \cap \Omega_{j}=\{\} \forall i \neq j .
$$

Depending on the problem and its spatial embedding, the $\Omega_{i}$ may all have different shapes (e.g. triangles or squares for two dimensional problems, or tetrahedrons, prisms or cuboids for three dimensional ones). However, for the ease of implementation and higher performance, often it is useful to use only one type of element. The following introduction is supposed to be abstract enough to be valid for all kinds of elements.

As a second step, the function $u(\mathbf{x})$ gets approximated as:

$$
u(\mathbf{x}, t) \approx u^{e}(\mathbf{x}, t)=\sum_{n} u_{n}(t) \phi_{n}(\mathbf{x}) .
$$

Where the trial functions $\phi_{n}(\mathbf{x}) \in V_{e}$ are taken from a finite dimensional approximation of the infinite dimensional function space where $u$ lives in:

$$
u \in H^{1}(\Omega) \rightarrow u^{e} \in V_{e} \subset H^{1}(\Omega) \quad \text { with } \quad H^{1}(\Omega)=\left\{v \in L^{2}(\Omega): \nabla v \in L^{2}(\Omega)\right\} .
$$

Here $H^{1}(\Omega)$ is a so called Sobolev space (for an introduction see e.g. [DW12] appendix $5)$. Then the goal is to select the time dependent coefficients $u_{n}(t)$ such that eq. (2.46) satisfies eq. (2.44). Inserting the approximation into eq. (2.44) yields:

$$
\sum_{n} \phi_{n}(\mathbf{x}) \partial_{t} u_{n}(t)-F\left(u^{e}, \mathbf{x}, t\right) \equiv R\left(\mathbf{x}, u_{1}(t), \ldots, u_{n}(t), t\right) \neq 0 .
$$


In the above equation, the left-hand side will generally not vanish and is therefore called the residual $R$. One way to define a system of equations for the unknown $u_{n}(t)$ is in terms of weighted integrals. Therefore, eq. (2.44) gets multiplied with a set of (linearly independent) test functions $v_{i}(\mathbf{x}) \in V_{e}$ taken from the same space as the functions $\phi_{n}$. Then one integrates over the domain $\Omega$ :

$$
\int_{\Omega} \mathrm{d} \mathbf{x} \mathrm{v}_{\mathrm{i}}(\mathrm{x}) \mathrm{R}\left(\mathrm{x}, \mathrm{u}_{1}(\mathrm{t}), \ldots, \mathrm{u}_{\mathrm{n}}(\mathrm{t}), \mathrm{t}\right)=0 \quad \forall \quad \mathrm{v} \in \mathrm{V}_{\mathrm{e}}
$$

The special choice of taking the $v_{i}$ from the same space as the $\phi_{n}$ is called Galerkin method. Inserting the definition of the residual from eq. (2.48) yields:

$$
\sum_{n} \frac{\partial}{\partial t} u_{n}(t) \int_{\Omega} \mathrm{d} \mathbf{x} \mathrm{v}_{\mathrm{i}}(\mathbf{x}) \phi_{\mathrm{n}}(\mathbf{x})=\int_{\Omega} \mathrm{d} \mathbf{x} \mathrm{v}_{\mathrm{i}}(\mathbf{x}) \mathrm{F}\left(\sum_{\mathrm{n}} \mathrm{u}_{\mathrm{n}}(\mathrm{t}) \phi_{\mathrm{n}}(\mathbf{x}), \mathbf{x}, \mathrm{t}\right) \quad \forall \quad \mathrm{v} \in \mathrm{V}_{\mathrm{e}}
$$

For many problems (i.e. definitions of $F(u, \mathbf{x}, t)$ ), it is possible to write

$$
\begin{aligned}
\left(u_{1}(t), \ldots, u_{n}(t)\right)^{T} & =\widetilde{\mathbf{u}}(t) \\
\int_{\Omega} \mathrm{d} \mathbf{x} \mathrm{v}_{\mathrm{i}}(\mathbf{x}) \phi_{\mathrm{n}}(\mathbf{x}) & =\mathbf{M} \\
\int_{\Omega} \mathrm{d} \mathbf{x} \mathrm{v}_{\mathrm{i}}(\mathbf{x}) \mathrm{F}\left(\sum_{\mathrm{n}} \mathrm{u}_{\mathrm{n}}(\mathrm{t}) \phi_{\mathrm{n}}(\mathbf{x}), \mathbf{x}, \mathrm{t}\right) & =\mathbf{A}(t) \widetilde{\mathbf{u}}+\mathbf{B} \widetilde{\mathbf{u}}+\mathbf{f}(\widetilde{\mathbf{u}})+\mathbf{k} .
\end{aligned}
$$

Hence eq. (2.50) essentially defines a (possibly nonlinear) system of coupled ordinary differential equations. It is called the semi-discrete description and with eqs. (2.51)(2.53) it can be rewritten as:

$$
\mathbf{M} \frac{\partial}{\partial t} \widetilde{\mathbf{u}}(t)=\mathbf{A}(t) \widetilde{\mathbf{u}}+\mathbf{B} \widetilde{\mathbf{u}}+\mathbf{f}(\widetilde{\mathbf{u}})+\mathbf{k}
$$




\subsubsection{Temporal discretization: 3 -stage linearly implicit Runge-Kutta}

In general an $s$-stage implicit Runge-Kutta integration schema for a differential equation $y^{\prime}=f(t, y)$ is defined by the following equations ${ }^{6}$ :

$$
\begin{aligned}
y_{n+1} & =y_{n}+\sum_{i=1}^{s} b_{i} k_{i} \\
k_{i} & =h f\left(t_{n}+\alpha_{i} h, y_{n}+\sum_{j=1}^{s} \alpha_{i j} k_{j}\right), i=1, \ldots, s \\
\alpha_{i} & =\sum_{j=1}^{i-1} \alpha_{i j} .
\end{aligned}
$$

Here, for the sake of readability, the size of the time step $\Delta t$ is denoted as $h$ and. For adaptive time steps $h$ will depend on the index $n$. Often, the required coefficients are written in a Butcher tableau:

$$
\begin{array}{c|ccccc}
0 & & & & & \\
\alpha_{2} & \alpha_{21} & & & & \\
\alpha_{3} & \alpha_{31} & \alpha_{32} & & & \\
\vdots & \vdots & & \ddots & & \\
\alpha_{s} & \alpha_{s 1} & \alpha_{s 2} & \ldots & \alpha_{s, s-1} & \\
\hline & b_{1} & b_{2} & \ldots & b_{s-1} & b_{s} \\
& b_{1}^{*} & b_{2}^{*} & \ldots & b_{s-1}^{*} & 0
\end{array}
$$

The last row with coefficients $b_{i}^{*}$ is optional and can be used to embed a second integration schema with lower stage $s-1$. The solution of the embedded stage can be used for error estimation.

The remaining part of the section will introduce an extension to the standard RungeKutta integration schema which is called a Rosenbrock-type method. Essentially, this method allows to integrate a system of ODEs of the form

$$
\hat{\mathbf{M}} \frac{d \mathbf{y}}{d t}=\mathbf{f}(t, \mathbf{y})
$$

without inverting the matrix $\hat{\mathbf{M}}$. Further, it is easy to implement and shows good numerical performance, because it removes the need to solve systems of nonlinear equations.

\footnotetext{
${ }^{6}$ Where $y$ and $f$ may also be vector valued quantities.
} 
For the derivation of this method the interested reader is referred to chapter IV.7 of [WH91]. Implementing the Rosenbrock schema is as simple as replacing eq. (2.56) with the following definition:

$$
k_{i}=h f\left(t_{n}+\alpha_{i} h, y_{n}+\sum_{j=1}^{s} \alpha_{i j} k_{j}\right)+h^{2} \gamma_{i} \frac{\partial f}{\partial t}\left(t_{n}, y_{n}\right)+h \frac{\partial f}{\partial y}\left(t_{n}, y_{n}\right) \sum_{j=1}^{i} \gamma_{i j} k_{j}
$$

As one can see, another matrix of coefficients $\boldsymbol{\Gamma}$ with $\gamma_{i}=\sum_{j=1}^{i} \gamma_{i j}$ is introduced. Solving the differential equation requires one more further transformation:

$$
\begin{aligned}
\mathbf{u}_{i} & =\sum_{j=1}^{i} \gamma_{i j} \mathbf{k}_{j} \\
\mathbf{y}_{n+1} & =\mathbf{y}_{n}+\sum_{j=1}^{s} m_{j} \mathbf{u}_{j} \quad \text { with }\left(m_{1}, \ldots, m_{s}\right)=\left(b_{1}, \ldots, b_{s}\right) \boldsymbol{\Gamma}^{-1}
\end{aligned}
$$

One ends up with implicit and possibly nonlinear equations for the $\mathbf{u}_{i}$ :

$$
\left(\frac{1}{h \gamma_{i i}} \hat{\mathbf{M}}-\frac{\partial \mathbf{f}}{\partial \mathbf{y}}\left(t_{n}, \mathbf{y}_{\mathbf{n}}\right)\right) \mathbf{u}_{i}=\mathbf{f}\left(t_{n}+\alpha_{i} h, \mathbf{y}_{n}+\sum_{j=1}^{i-1} a_{i j} \mathbf{u}_{j}\right)+\sum_{j=1}^{i-1} \frac{c_{i j}}{h} \mathbf{u}_{j}+\gamma_{i} h \frac{\partial \mathbf{f}}{\partial t}\left(t, \mathbf{y}_{n}\right)
$$

with the coefficient matrices $\left(c_{i j}\right)$ and $\left(a_{i j}\right)$ given as

$$
\begin{aligned}
\left(c_{i j}\right) & =\operatorname{diag}\left(\gamma_{i i}^{-1}, \ldots, \gamma_{s s}^{-1}\right)-\boldsymbol{\Gamma}^{-1} . \\
\left(a_{i j}\right) & =\left(\alpha_{i j}\right) \boldsymbol{\Gamma}^{-1}
\end{aligned}
$$

In the course of this work, a 3 -stage Rosenbrock schema with specific $\alpha_{i j}, b_{i}, b_{i}^{*}$ and $\gamma_{i j}$ called ROWDA3 [Roc87] will be used:

$$
\begin{array}{c|ccc}
0 & & & \\
\alpha_{2} & \alpha_{21} & & \\
\alpha_{3} & \alpha_{31} & 0 &
\end{array} \quad \text { and } \Gamma=\left(\begin{array}{ccc}
\gamma & 0 & 0 \\
\gamma_{21} & \gamma & 0 \\
\gamma_{31} & 1 & \gamma
\end{array}\right)
$$

The original and transformed coefficients are given in table 2.1. The choice of coefficients renders eq. (2.63) into a linear system, and the embedded 2-stage schema can be solved with minimal overhead. 


\begin{tabular}{c|c||c|c} 
& original & & transformed \\
\hline$\alpha_{21}$ & 0.7 & $a_{21}$ & 1.605996252195329 \\
$\alpha_{31}$ & 0.7 & $a_{31}$ & 1.605996252195329 \\
$\alpha_{32}$ & 0 & $a_{32}$ & 0 \\
$b_{1}$ & 0.3197278911564624 & $m_{1}$ & 2.236727045296589 \\
$b_{2}$ & 0.7714777906171382 & $m_{2}$ & 2.250067730969645 \\
$b_{3}$ & -0.0912056817736006 & $m_{3}$ & -0.2092514044390320 \\
$b_{1}^{*}$ & 0.926163587124091 & $m_{1}^{*}$ & 2.059356167645941 \\
$b_{2}^{*}$ & 0.073836412875909 & $m_{2}^{*}$ & 0.1694014319346527 \\
$b_{3}^{*}$ & 0 & $m_{3}^{*}$ & 0 \\
$\gamma$ & 0.435866521508459 & $c_{i i}$ & 0 \\
$\gamma_{21}$ & 0.1685887625570998 & $c_{21}$ & 0.8874044410657823 \\
$\gamma_{31}$ & 4.943922277836421 & $c_{31}$ & 23.98747971635035 \\
$\gamma_{32}$ & 1 & $c_{32}$ & 5.263722371562130 \\
\hline
\end{tabular}

Tab. 2.1: Coefficients for the integration schema. The left column denotes the variables from [Roc87] and the right column gives their corresponding transformed values according to eqs. (2.62), (2.64) and (2.65). 


\section{Chapter 3}

\section{Biology and Models}

In this chapter, a very brief introduction to the biology of eukariotic cells and the general aspects of modeling of intracellular processes will be given. Then it will continue to describe the cellular subsystem this work is related to with a special focus on the $\mathrm{IP}_{3} \mathrm{R}$ in section 3.4. The descriptions of the HDM and CGM will be contained in their own respective chapters.

To understand the following sections, the reader has to be aware of some very rudimentary facts about cell physiology, some of which are:

- What are cells made of? How are they organized?

- How does transport work within cells?

- How do cells communicate internally and with other cells?

The first question is best answered with help of fig. 3.1, a schematic view of some eukaryotic cell. One immediately grasps that there is lots of complexity involved. The most important aspects are: The plasma membrane separating the interior of the cell from its exterior. It confines molecules and proteins to the interior of the cell. Transport through the membrane has to involve some special process, e.g. via ion channels located in the membrane or via secretory vesicles. Further, the interior of the cell contains some large structures called organelles (e.g. the mitochondria and the nucleus). The remaining space within the cell is filled with the cytoskeleton (actin filaments) and the cytosol. ${ }^{1}$ The cytosol is a mix of various different size proteins, water, and other smaller molecules which constitute to the cells supply with nutrition, messaging, and recycling processes.

The transport mechanisms within a cell can be categorized into two different cases:

\footnotetext{
${ }^{1}$ The term cytoplasm refers to everything within the cell excluding only the nucleus.
} 


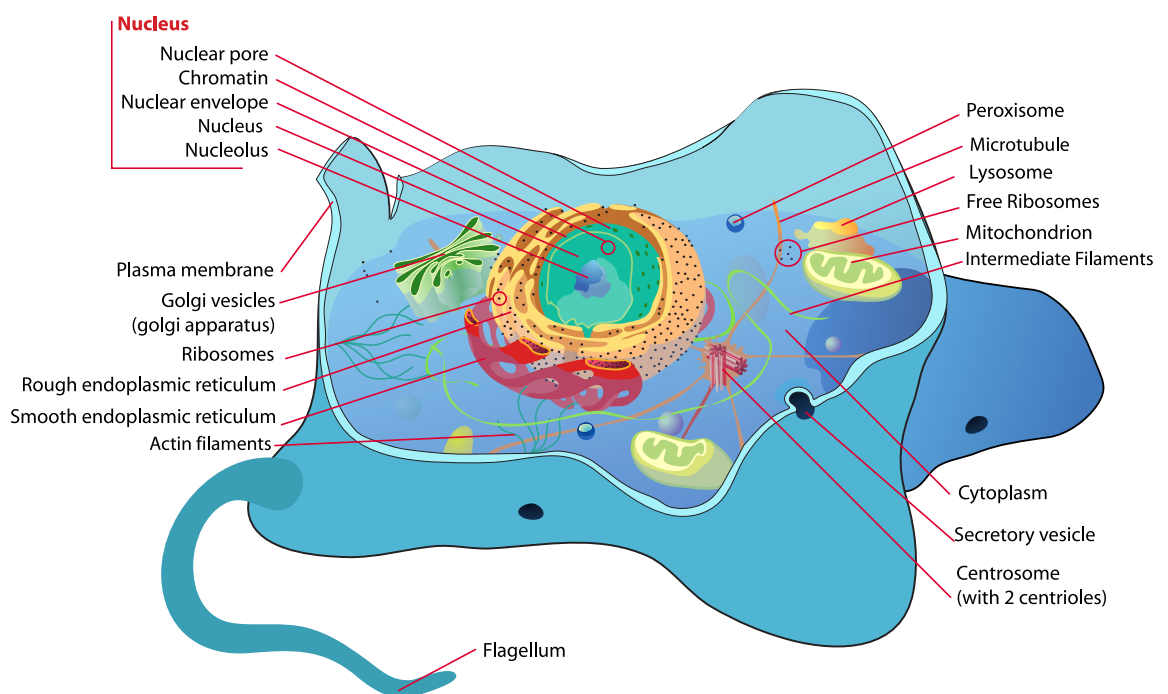

Fig. 3.1: Schematic of some eukaryotic cell. The interior of the cell is separated from the exterior via the plasma membrane. The interior of the cell is filled with organelles and cytoplasm which is a mixture of a vast amount of different molecules, proteins, nutrition and other cell byproducts. The organelles are substructures which have specialized tasks and may be separated from the cytoplasm with their own membrane. By LadyofHats (Mariana Ruiz) [Public domain], via Wikimedia Commons.

- Active transport. Specialized proteins called molecular motors can carry substances through the cell. Often those motors walk along the actin filament network within the cell.

- Passive transport. Molecules and proteins move randomly within the cell because of diffusion. Diffusive movement is slower for large proteins and fast for smaller molecules. Also the diffusion process within a cell is not necessarily normal diffusion, but can in general be anomalous diffusion due to the crowdedness and variety of the cytosol.

Finally communication in cells often takes place in form of signaling cascades. A signaling cascade can be thought of as a chain reaction, where a reaction between some molecules yields a product which in turn triggers further reactions of other molecules. Because of the transport mechanisms described above, a cascade can trigger further reactions in some remote part of the cell. One concludes that the transport mechanisms are required for many signaling pathways, and that signaling pathways in turn can control transport mechanisms. 


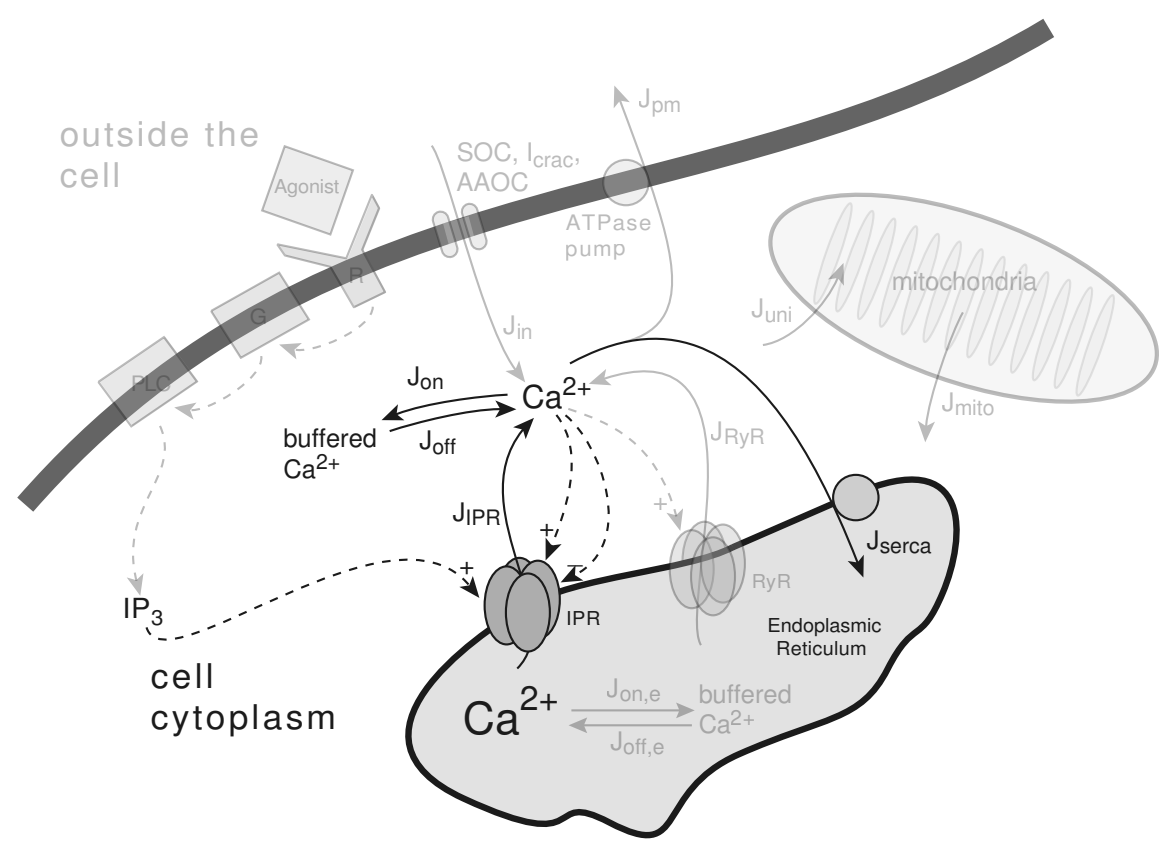

Fig. 3.2: A incomplete schema of agonists involved in calcium signaling. Entities that are not topic of this work are grayed out. Figure taken from [KS10].

\subsection{Calcium signaling: the system of interest}

After the rather general and abstract introduction, the focus now turns to the role of $\mathrm{Ca}^{2+}$ within a cells physiology. The $\mathrm{Ca}^{2+}$ signaling pathway on its own still remains to be a very complex process involving a variety of different secondary messenger proteins. Figure 3.2 shows part of this pathway and also depicts which processes will be investigated further within this work. The textbook [KS10, chapter 7] provides a nice reference for this topic, and its arguments will be partially followed here. Notably the introduction of [KS10, Chapter 7] provides a comprehensive overview on review articles related to the physiological relevance of the $\mathrm{Ca}^{2+}$ signaling pathways, at this point, this overview can be extended to include [Ber14], providing an extensive discussion on the role of $\mathrm{Ca}^{2+}$ and $\mathrm{IP}_{3}$ in neuronal signaling. In this work, the following participants of the $\mathrm{Ca}^{2+}$ signaling pathway will continue to be of particular interest:

- Endoplasmic reticulum (ER) an organelle which holds vast amounts of $\mathrm{Ca}^{2+}$ and is separated from the cytoplasm via the endoplasmic reticulum (ER) membrane. It is usually located near the nucleus and forms two different types, the rough and the smooth ER (see fig. 3.1). An interesting property in the context of this work is the reticular structure of the ER: its membrane may form spatial 
pockets, where diffusion is confined in one or two dimensions. Figure 3.3 shows a $3 \mathrm{D}$ reconstruction of electron microscopy images taken from [Ter +13$]$ depicting the folded structure.

- $\mathrm{IP}_{3}$ receptors $\left(\mathrm{IP}_{3} \mathrm{R}\right), \mathrm{Ca}^{2+}$ ion channels which are regulated via inositol 1,4,5trisphosphat $\left(\mathrm{IP}_{3}\right)$, a secondary messenger molecule (see fig. 3.4). $\mathrm{IP}_{3} \mathrm{R}$ are usually found at ER or sarcoplasmic reticulum (SR) membranes. They are selective for a specific type of ion $\left(\mathrm{Ca}^{2+}\right.$ in this case) and allow transport of ions along the gradient of concentration. Besides the binding cite for $\mathrm{IP}_{3}$ each of the four monomers of $\mathrm{IP}_{3} \mathrm{R}$ possesses an activating and an inhibitory $\mathrm{Ca}^{2+}$ binding site [SL10]. A structural reconstruction of $\mathrm{IP}_{3} \mathrm{R}$ is presented in fig. 3.7, a good overview over the structural properties can be found in [SL10].

- Sarcoplasmic/endoplasmic reticulum calcium ATPase (SERCA), $\mathrm{Ca}^{2+}$ pumps which pump $\mathrm{Ca}^{2+}$ from the cytoplasm to the ER or SR. Because they operate against the $\mathrm{Ca}^{2+}$ gradient, they require energy in form of adenosine triphosphate (ATP).

- $\mathrm{Ca}^{2+}$ buffers, including intrinsic buffers of the cell as well as exogenous buffers like Ethylene glycol tetraacetic acid (EGTA) and 1,2-bis(o-aminophenoxy)ethaneN,N,N',N'-tetraacetic acid (BAPTA). Buffers are usually characterized by their effective concentration (meaning the number of $\mathrm{Ca}^{2+}$ ions they can bind per unit volume) and their binding rates (on and off). Some type of special exogenous buffers are so called dye buffers. These buffers change their fluorescence properties upon $\mathrm{Ca}^{2+}$ binding and are therefore often used in experiments to indirectly measure the free $\mathrm{Ca}^{2+}$ concentration. Some values are given in table 5.1. ${ }^{2}$

- $\mathrm{Ca}^{2+}$ itself. Intracellular resting $\left[\mathrm{Ca}^{2+}\right]$ resides in the range of few tenth or hundredth of $\mu \mathrm{M}$. In contrast to that, the extracellular $\left[\mathrm{Ca}^{2+}\right]$ usually is on the order of $\mathrm{mM}$ [KS10]. Usually, the fast majority of $\mathrm{Ca}^{2+}$ ions are bound to buffers both in the luminal (inner ER), as in the cytosol. Still, the free $\left[\mathrm{Ca}^{2+}\right]$ within the ER may reach up to a few hundred $\mu \mathrm{M}$ to $1 \mathrm{mM}$ [MP98; Fal04]. [KS10]

The steep gradients between the $\left[\mathrm{Ca}^{2+}\right]$ within the different compartments are only possible due to the impermeability of the plasma membrane and various ion pumps, which transport $\mathrm{Ca}^{2+}$ ions against that gradient back into the $\mathrm{Ca}^{2+}$ stores. The large gradients allows a cell to react very sensitive to marginal changes of $\left[\mathrm{Ca}^{2+}\right]$ and are essential for passive release mechanisms.

\footnotetext{
${ }^{2}$ As discussed in [Lyt+92] ATP can also buffer $\mathrm{Ca}^{2+}$, however, this buffering is assumed to be negligible when calcium concentration $\left(\left[\mathrm{Ca}^{2+}\right]\right)$ is only in the $\mu \mathrm{M}$ scale.
} 

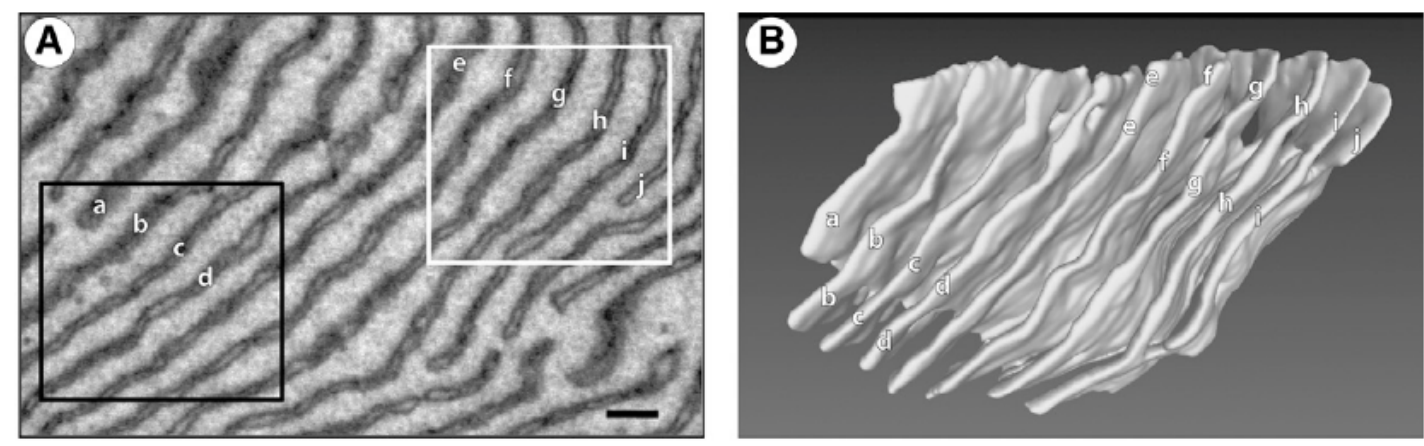

Fig. 3.3: Electron microscopy slice (A) of the ER membrane and a corresponding 3D reconstruction (B) from [Ter+13]. The scale bar corresponds to $200 \mathrm{~nm}$.

One important effect in this context is calcium induced calcium release (CICR). Because the $\mathrm{IP}_{3} \mathrm{R}$ has a positive feedback on $\mathrm{Ca}^{2+}$, small amounts of $\mathrm{Ca}^{2+}$ which enter the cytosol can result in a large $\mathrm{Ca}^{2+}$ release from some of the internal $\mathrm{Ca}^{2+}$ store (e.g. the ER) if the primary $\mathrm{Ca}^{2+}$ reaches one of the $\mathrm{IP}_{3} \mathrm{Rs}$. Further, because of the inherent noise, $\mathrm{IP}_{3} \mathrm{R}$ may just open by chance and hence may trigger other $\mathrm{IP}_{3} \mathrm{Rs}$ which are nearby. Besides some other regulatory mechanisms, this positive feedback is counteracted by two modulations of the $\mathrm{IP}_{3} \mathrm{R}$ : each $\mathrm{IP}_{3} \mathrm{R}$ also allows for inhibitory $\mathrm{Ca}^{2+}$ binding and is further modulated via it's primary messenger molecule $\mathrm{IP}_{3}$. This results in a large variety of different release patterns which will be depicted in the next section.

\subsection{The zoo of calcium patterns}

\section{Blibs}

Blibs are the most simple pattern of $\mathrm{Ca}^{2+}$ release events. Every single channel opening, which does not trigger other channels directly via CICR is called a blib. Depending on the experimental setup, blibs can be detected for individual channels, as well as for clusters of channels. While the term blib is usually used for fluorescence measurements, patch clamp recordings of individual channels can give insight into a single channels dynamics. For example, the binding configuration of a single $\mathrm{IP}_{3} \mathrm{R}$ and $\mathrm{CICR}$ can already result in bursts of blibs. Further $\mathrm{IP}_{3} \mathrm{Rs}$ have multiple different conductance levels, and hence the release amplitudes may vary from blib to blib [MF97]. The spatial and temporal scale of blibs is in the order of $10 \mathrm{~nm}$ to $100 \mathrm{~nm}$ and a few milli-seconds, respectively [Rud14b]. 

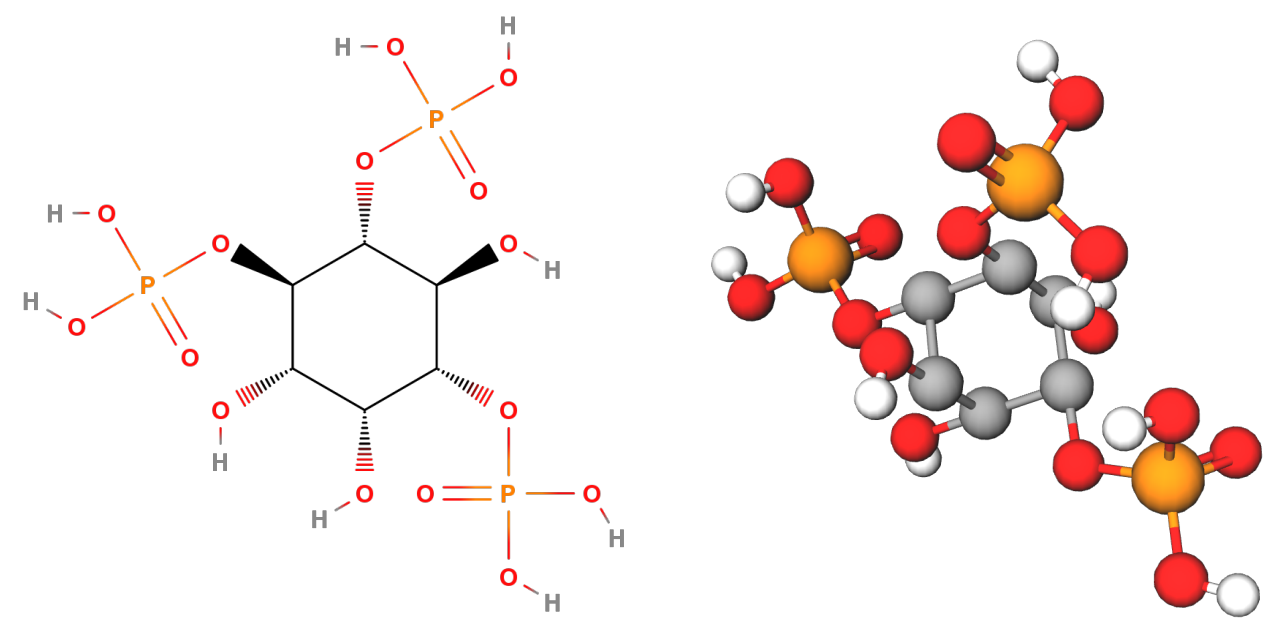

Fig. 3.4: Structure of Inositol 1,4,5-trisphosphat $\left(\mathrm{IP}_{3}\right)$. Rendered with: http: //molview. org.

\section{Puffs and Sparks}

As reported e.g. in [Tau+09], lone $\mathrm{IP}_{3} \mathrm{R}$ can have a tendency to group together to clusters of a couple of receptors. In these groups, the tight coupling via CICR can lead to synchronized release of multiple channels [DSP12; SP09]. Depending on where this behavior is observed, such a collective release event is called puff or spark. The term puff is usually used in the context of $\mathrm{IP}_{3} \mathrm{R}$ while for some cell types such as myocytes, or when referring to release via the similar Ryanodine receptor (RyR) the term spark is employed [RLS11]. As the positive feedback between clustered channels is not necessarily fail-safe, puffs strongly vary in amplitude as showed in [SP09] (see fig. 3.5). This also means that a sequence of puffs may be interspersed with blibs of individual channels. Puffs usually last up to a couple of $100 \mathrm{~ms}$ and the size of clusters is in the range of $500 \mathrm{~nm}$ [Rud14b].

\section{Waves}

If the physiological conditions allow CICR to overcome the much larger distance between individual clusters, then the release pattern may emerge into waves which can cover many micro meters [MP01; MCP99]. In very large cells, the waves may even show complex spatio temporal behavior such as spirals [Atr+93]. The transition from puffs to waves can be disrupted by adding artificial $\mathrm{Ca}^{2+}$ buffers as presented in [CP00]. Further, as depicted in fig. 3.5, the hierarchical structure repeats, as waves may be 

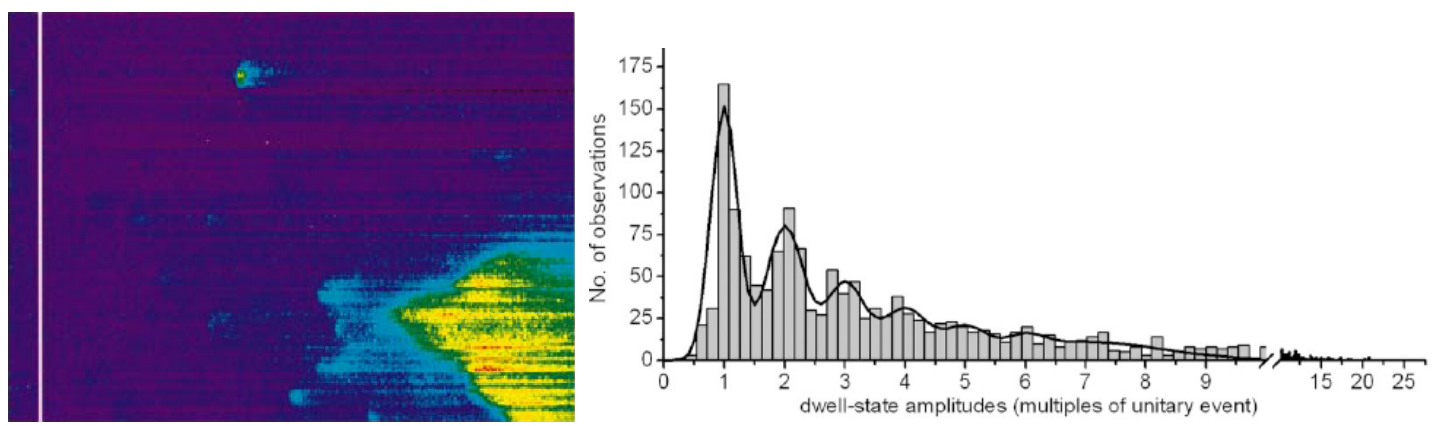

Fig. 3.5: Left: A randomly occurring wave with some preceding puffs after photo uncaging of $\mathrm{IP}_{3}$. Taken from [MCP99][Fig. 3]. Right: The distribution of fluorescence amplitudes of puff sites. Taken from [SP09][Fig. 4].

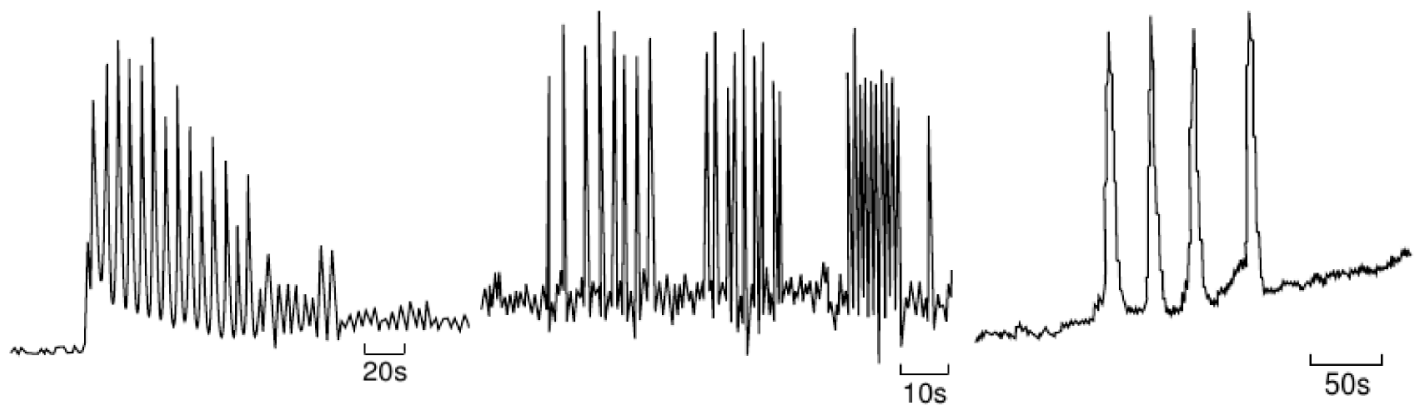

Fig. 3.6: Global calcium oscillations for different cell types with different stimuli. Taken from [KS10] [Fig. 7.2].

interspersed with puffs at some of the clusters [Niv+13; MCP99; MP01].

\section{Global oscillations}

If the release of clusters of $\mathrm{IP}_{3} \mathrm{R}$ synchronizes, then the propagating aspect of waves vanishes and global oscillations of $\mathrm{Ca}^{2+}$ take over [CT03]. As can be seen from fig. 3.6, a multitude of different oscillations can be observed.

\subsection{Modeling}

Very similar to a theory, a model is the mathematical description of an idea how some observed process may work. A "good" model is designed such that it reflects the observations and allows to predict yet unobserved properties of the process. Further, while proven "wrong" by some false predictions a model may remain useful by providing 
an intuitive explanations for many of the observations.

The models presented in this work aim to describe the patterns introduced in the previous section (section 3.2). Therefore they need to incorporate several different aspects:

- ion channel gating (e.g. changes in conductance of ion channels)

- transport mechanisms (e.g. diffusion of $\mathrm{Ca}^{2+}$ and active transport via SERCA)

- chemical reactions (e.g. binding of $\mathrm{Ca}^{2+}$ to buffer proteins)

The feedback between these aspects makes modeling such joint systems a challenging task. As in many other fields where complex coupled systems are investigated it makes sense to first study separated subsystems without feedback, which may involve some "driving mechanism" representing the outside of the subsystem. ${ }^{3}$ Therefore, the remaining part of this section will be attributed to the vast amount of different models available in literature. A great overview over the available techniques is also given in [Dup+11][Table 1] and [Rud14b][Table 2]. After this, the chapter will conclude with the channel gating model which will be used throughout this work.

\subsubsection{Channel gating models}

Many models use continuous gating variables to describe the gating of ion channels. In this case, the conductance of one or many ion channels is described by dynamic variables which evolve according to a Langevin equation (see section 2.3). This allows to easily incorporate noise effects, as well as feedback between variables (e.g. between $\mathrm{Ca}^{2+}$ and the open fraction of a cluster in CICR). Further, such models allow to apply methods from the field of dynamical systems, like bifurcation analysis (see e.g. [SUJ09]), which are known to work well in theoretical neuroscience. One example for this approach is given in [SJ02] which uses the Li-Rinzel model [LR94] and concludes that clustering of $\mathrm{IP}_{3} \mathrm{R}$ improves the sensitivity for small $\mathrm{Ca}^{2+}$ stimuli.

While computationally simple, continuous gating variables bring two major drawbacks: first, noise may lead to negative values of the dynamic variable. This is difficult to interpret when the variable describes an occupation number that must not be negative. Hence, a special treatment of noise is required to avoid such situations. Second, if the system of interest is small, which is the case if one is interested e.g. in the dynamics of a single cluster of ion channels, a continuous variable often gives a poor

\footnotetext{
${ }^{3}$ One great example from a completely different environment is software development. Modules of applications usually get tested against a fake environment which emulates the real world for a given module.
} 
approximation since quantization effects may have an important impact on the system. An interesting approach which tries to deal with these problems is presented in [Rud14a] where an "artificial" quantization is introduced into the continuous variable and comparisons to a discrete description are made.

Other models incorporate discrete random variables to describe the configuration of a whole cluster of $\mathrm{IP}_{3} \mathrm{R}$. E.g. in [Bar+00] a cluster is described by two occupation numbers which define how many of the clusters channels are open. Shuai et. al. follow a similar approach based on the Li-Rinzel model [LR94] which yields three different occupation numbers. They investigate the reliability of wave propagation in a $2 \mathrm{D}$ grid of clusters where the number of open channel in each cluster evolves according to a master equation [SJ03].

More detailed models keep track of individual channel states by evolving a master equation per channel, as done in [SUJ09] where statistical properties of individual puffs are investigated. This is the approach which will be pursued within this work and the used DeYoung-Keizer model [DK92] will be introduced in section 3.4.

\subsubsection{Transport and chemical reactions}

One way to describe transport is on a microscopic level, where one tracks the positions (and possibly orientation) of all relevant molecules of the system. Examples for this approach are Markov chain Monte Carlo (MCMC) methods and molecular dynamics simulations. These methods allow a detailed insight into the ongoing processes and may provide a very realistic system. However, they require a very detailed description of e.g. initial conditions and the molecular interactions of involved proteins. Further, the high level of detail results in an immense computational complexity which usually restricts the system of interest to a spatial extent at the nanometer scale and simulated time intervals to a couple of microseconds [CDS16]. An interesting work by R. Erban et. al. avoids lots of computational effort by using two different flavors of a microscopic description. In their work, they use a occupation number description for grid cells of regions which are considered non critical, and microscopic simulations for regions where a high level of detail is required [FRE13].

As an alternative, one can make use of statistical mechanics and describe the system as continuous. In this case one drops the information per particle and instead describes many particles via collective properties, e.g. their concentration. Transport can then be described by a diffusion equation or in case of drift with it's generalization, the Smoluchowski equation (sometimes also called Fokker-Plank or Kolmogorov forward equation because it describes a probability density). For a derivation of these continuous equations from a microscopic description see e.g. [Pat96][chapter $14.3-14.5$ ]. 
In principle, modeling of chemical reactions can be discriminated in the same way as modeling of transport by adding some additional treatment for the reaction processes. E.g. for a microscopic description of molecules, reactants may consume each other and get removed from the system, while the resulting products have to be inserted into the system. On the other hand, for continuous descriptions, each reactant yields is own continuous equation, which is coupled to the other reactants via reaction terms.

One important difference between the microscopic and the statistical mechanics description is the presence of noise. While noise is an essential ingredient in statistical mechanics, the resulting macroscopic equations are deterministic. I.e. the evolution of the macroscopic system is fully defined by it's governing equations and it's initial state, whereas the microscopic system evolves randomly. In some sense, the absence of noise is a major drawback of the continuous description since it requires "mathematical glue" to be coupled to the microscopic description of ion channels. As investigated in [FRE13] the absence of noise in the macroscopic description may lead to significantly different results if concentrations of particles are low. This goes in hand with the difficult interpretation of low concentrations and their corresponding number of particles within a small volume: E.g. $c_{0}=0.02 \mu \mathrm{M}$ corresponds to $\approx 12 \mathrm{Ca}^{2+}$ ions per $1 \mathrm{\mu m}^{3}$, however the binding region of a $\mathrm{IP}_{3} \mathrm{R}$ may only have an extent of $10^{3} \mathrm{~nm}^{3}$. How does one interpret $0.000012 \mathrm{Ca}^{2+}$ ions?

The continuous description in general yields three dimensional PDEs which are again rather complicated to deal with. Hence additional approximations are frequently used to further reduce the model complexity. E.g. it often makes sense to divide the system of interest into different "levels of localization", some examples of which are:

- A single channel's close vicinity. This region will dominate the channel's protein binding and hence its gating probabilities.

- The domain of a cluster of channels. This domain may be required to mediate coupling between channels of a cluster.

- Some functional regions withing a cell (e.g. dendritic spines in neurons).

- The whole cellular volume may provide a sufficient level of detail for models dealing only with inter cellular signaling.

Separating into those compartments often reduces the system of PDEs to a system of coupled ODEs. Essentially the same approach is followed in the FEM, where some artificial regions are defined such, that spatial gradients of concentrations will get resembled with good accuracy (see section 5.1 andsection 2.5.1). Dividing the system 


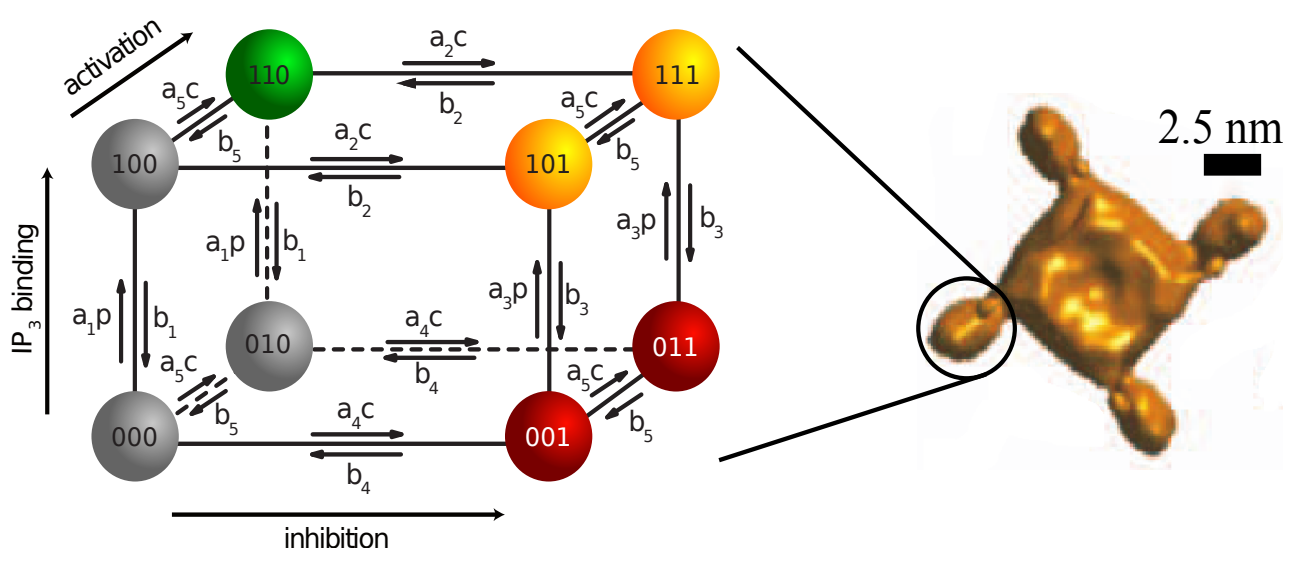

Fig. 3.7: Left: Gating scheme of the modified DYK model used to model the states of a single subunit. The transition rates are determined by the $\left[\mathrm{Ca}^{2+}\right]$ and $\left[\mathrm{IP}_{3}\right]$ here denoted by $c$ and $p$, respectively. Right: Single-particle cryo-EM reconstruction of the $3 \mathrm{D}$ structure of $\mathrm{IP}_{3} \mathrm{R}$ from $[\mathrm{Jia}+02]$. One can clearly see the tetrameric structure of the $\mathrm{IP}_{3} \mathrm{R}$, where each of the monomers will be resembled by the eight state gating schema on the left.

into different domains also allows to apply different models for the individual domains as done in e.g. [FRE13] or as presented in section 6.1.

For a model (reaction and transport) involving different time scales, steady state approximations will also greatly reduce the model's complexity since they allow to get rid of some of the fast evolving dynamic variables. Examples for this are the analytic rapid buffer approximation (RBA) derived in [Smi+01] for buffered calcium release from a single channel, "instantaneous transport" as done in [GS08], or an approximation for the channel current in [BF07]. This approach will also be followed in section 6.1.

Other approximations allow for an analytic description in the limit of very large concentrations (e.g. excess buffer approximation [Smi+01]), or even analytic approximations for $\mathrm{Ca}^{2+}$ concentrations around a single cluster of $\mathrm{IP}_{3} \mathrm{R}$, which, carefully coupled to an ion channel gating model allow to study spontaneous waves and backfiring [FTL00]. Finally, if one assumes spherical symmetry, once can simplify a three dimensional system to one radial dimension as done in [Ull+12].

\subsection{DeYoung-Keizer model for IP3R channel gating}

The original DeYoung-Keizer (DYK) model [DK92; KD94] consists of three individual and independent subunits where each subunit possesses three different binding sites: An activating site for $\mathrm{Ca}^{2+}$, an inhibiting $\mathrm{Ca}^{2+}$ site, and an $\mathrm{IP}_{3}$ binding site. Thus, each subunit possesses eight different configurations and twelve distinct transitions 
between those configurations which are all depicted in fig. 3.7. Because the subunits are indistinguishable, it makes sense to combine the state of the subunits into a $2 \times 2 \times 2$ tensor of occupation numbers $\mathrm{X}_{i j k}$ where the indices $i, j$, and $k$ indicate the states of the $\mathrm{IP}_{3}$ site, the activating $\mathrm{Ca}^{2+}$ site, and the inhibiting $\mathrm{Ca}^{2+}$ site, respectively. An index is 1 if $\mathrm{Ca}^{2+}$ or $\mathrm{IP}_{3}$ are bound and 0 if not. A channel is considered open if the activating $\mathrm{Ca}^{2+}$ and the $\mathrm{IP}_{3}$ binding sites of all three subunits are occupied, i.e. if $X_{110}=3$. In the original work, the transition rates between states were fit to experimental data for the steady state open probability and $\mathrm{IP}_{3}$ binding data from [LJE91] and [JRW89], respectively. In [LJE91] it was shown, that the steady-state open probability $P_{o}$ can be well fit as a function of the calcium concentration $c$ with

$$
P_{o}(c) \propto\left[\frac{c+k}{(c+K)(c+k)}\right]^{m}
$$

where $m=2.7$ and $K=k=0.2 \mu \mathrm{M}$. The above fit also matches with the analytic expression for $P_{o}$ derived in [DK92].

In this work, a slightly modified variant of the DYK model is used. ${ }^{4}$ It was first introduced in [FTL00] and is motivated by the tetrameric structure of the $\mathrm{IP}_{3} \mathrm{R}[\mathrm{Jia}+02$; SL10]. Here the $\mathrm{IP}_{3} \mathrm{R}$ channel consists of four identical subunits. For the channel to be open, three out of four subunits have to be in the 110 state. Further, the parameters defining the transition rates of the model were fitted to patch-clamp data for type 1 $\mathrm{IP}_{3} \mathrm{R}$ channels in [Fos+07; Shu+09; MMF98] and are given in table 3.1. The parameter set in the upper part of the table corresponds to the values used in [Ruc+15]. With exception of $d_{4}$ (and consequently $d_{3}$ ), they match the rates of an earlier publication [RJS12]. The values $d_{3}$ and $d_{4}$ have been adjusted by a factor of about 3 to reflect a large shift in open probability in dependence on $\left[\mathrm{IP}_{3}\right]$. The lower part of the table depicts the rates which were used in [RR16]. There $a_{1}, b_{1}, a_{4}$ and $b_{4}$ where increased by a factor of 10 . It can be argued, that this is legitimate, since the rates $a_{4}\left(b_{4}\right)$ are in general more difficult to assess than the dissociation coefficients $d_{i}$. Further, increasing $a_{1}\left(b_{1}\right)$ should provide a better match for the fast response after a $\mathrm{IP}_{3}$ step increase which was observed in [Mak+07] and [SRP06]. In this work, all sets of transition rates satisfy detailed balance:

$$
d_{1} d_{2}=d_{3} d_{4}
$$

Since a channel cannot open if two or more subunits lack $\mathrm{IP}_{3}$, the channel is rendered unactivatable, because the remaining two subunits can never lead to a channel opening

\footnotetext{
${ }^{4}$ If not explicitly stated otherwise further on the term DYK model denotes this modified version. For a nice investigation on different variations of the DYK model, the interested reader is referred to $[\mathrm{Shu+09]}$.
} 
according to the criterion given above. Vice versa, a channel is called activatable, if at least three of its subunits have bound $\mathrm{IP}_{3}$. Note that this definition allows that an inhibited channel may still be activatable (e.g., two subunits in $\mathrm{X}_{110}$ and two subunits in $\mathrm{X}_{111}$ ).

Looking at fig. 3.7 one does notice that binding of $\mathrm{IP}_{3}$ depends on whether a subunit is inhibited or not: if the channel is inhibited (right side of cube) the equilibrium number of subunits with bound $\mathrm{IP}_{3}$ is lower than on the left hand side, because the dissociation coefficient $d_{3}$ is larger than $d_{1}$ (see table 3.1$)^{5}$. While the rate of $\mathrm{IP}_{3}$ unbinding $b_{1}$ is extremely slow for a resting channel, unbinding $\mathrm{IP}_{3}$ from inhibited channels operates on the same time scale as "de-inhibiton" $\left(b_{3} \approx b_{2}\right)$. Hence, it is more likely for a subunit to move to an unbound $\mathrm{IP}_{3}$ state via an inhibited state.

Ultimately, one can imagine the following cycle: A channel opens, gets inhibited, loses $\mathrm{IP}_{3}$ and then slowly recovers into an activatable state from where it can open again. While the loss of $\mathrm{IP}_{3}$ is promoted by inhibition, it still has to be distinguished from normal inhibition since it only represents a secondary effect and does not directly interrupt the CICR feedback loop. A detailed investigation of the $\mathrm{IP}_{3}$ unbinding process can be found in the result sections.

Figure 3.8 shows the steady state open probability $P_{o}$ for the modified DYK model according to eqs. (3-8) from [Shu+09] as a function of $\mathrm{Ca}^{2+}$ and $\mathrm{IP}_{3}$ concentration with the parameters from table 3.1. One can clearly see a shift of the maximum probability to higher $\mathrm{Ca}^{2+}$ concentrations for increasing $\mathrm{IP}_{3}$. This shift and the increase of open probability with $\left[\mathrm{IP}_{3}\right]$ are of key importance for the findings presented in section 5.3 and section 6.2 .

\footnotetext{
${ }^{5}$ Here, the term equilibrium denotes an equilibrium only for "vertical" transitions, i.e. transitions of the $\mathrm{IP}_{3}$ binding site.
} 

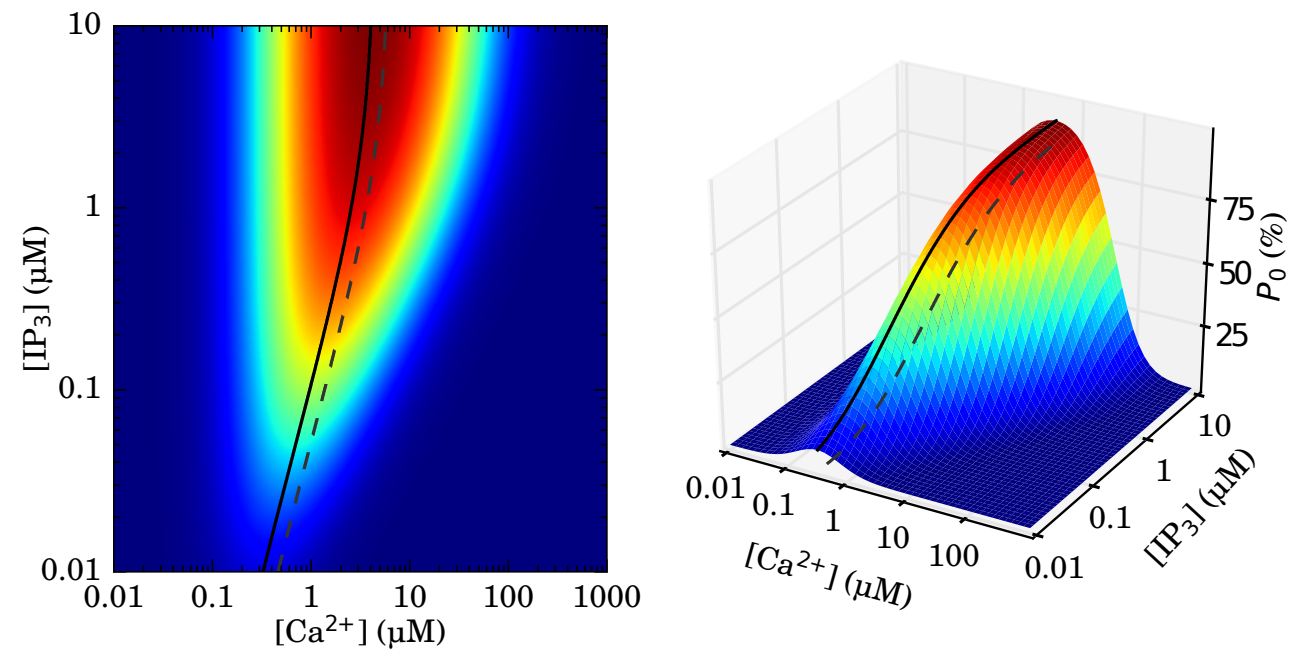

Fig. 3.8: Steady state open probability of the modified DYK model for a single channel for the upper set of parameters from table 3.1 . For increasing $\left[\mathrm{IP}_{3}\right]$, the open probability increases and the maximum of the open probability shifts to higher $\left[\mathrm{Ca}^{2+}\right]$. The lines denote the position of the maximum open probability depending on $\left[\mathrm{IP}_{3}\right]$. The gray dashed line corresponds to the second parameter set from table 3.1 .

\begin{tabular}{|c|c|c|c|c|}
\hline & $i$ & $a_{i}$ in $1 / \mu \mathrm{Ms}$ & $b_{i}$ in $1 / \mathrm{s}$ & $d_{i}$ in $\mu \mathrm{M}$ \\
\hline $\mathrm{IP}_{3}$ while not inhibited & 1 & 0.20 & $2 \times 10^{-4}$ & 0.001 \\
\hline Inhibition with $\mathrm{IP}_{3}$ & 2 & 0.02 & 1.56 & 78 \\
\hline $\mathrm{IP}_{3}$ while inhibited & 3 & 0.40 & 0.80 & 2 \\
\hline Inhibition without $\mathrm{IP}_{3}$ & 4 & 0.10 & $3.9 \times 10^{-3}$ & 0.039 \\
\hline Activation & 5 & 100 & 25 & 0.25 \\
\hline $\mathrm{IP}_{3}$ while not inhibited ${ }^{1}$ & 1 & 2 & $2 \times 10^{-3}$ & 0.001 \\
\hline Inhibition without $\mathrm{IP}_{3} 1$ & 4 & 1 & $3.9 \times 10^{-2}$ & 0.039 \\
\hline
\end{tabular}

Tab. 3.1: Channel gating parameters $a_{i}, b_{i}$ and the dissociation coefficient $d_{i}=b_{i} / a_{i}$ of the modified DYK model. The upper part resembles the parameters used in [Ruc +15$]$ and section 5.3. The lower part depicts the changes of parameters introduced in section 6.2 and [RR16]. There, $a_{1}, b_{1}, a_{4}$, and $b_{4}$ were increased by a factor of 10 . 


\section{Chapter 4}

\section{Methods}

\subsection{Hybrid schema}

In section 3.4 of the previous chapter, the DeYoung-Keizer (DYK) model was introduced as a discrete state stochastic channel gating model. The next two chapters will introduce the HDM and CGM, which both contain a deterministic description of the $\mathrm{Ca}^{2+}$ evolution. As depicted by fig. 4.1, this means that both joint systems HDM plus DYK, and CGM plus DYK consist of a mixed discrete and continuous state as described in section 2.4. Unfortunately, as explained in that section, solving the equation for the composed state is not feasible, which leaves MCMC sampling as the mean of choice to investigate the evolution of such systems. This section will discuss the algorithm used to perform the MCMC sampling.

First, recall the two starting points of section 2.4, eqs. (2.24) and (2.25):

$$
\begin{gathered}
\frac{\mathrm{d} c_{k}}{\mathrm{~d} t}=f_{k}(\mathbf{c}, t)+g_{k}(\mathbf{c}, S(t), t) \\
\frac{\mathrm{d} P\left(S(t)=S_{n}\right)}{\mathrm{d} t} \equiv \frac{\mathrm{d} \rho_{n}}{\mathrm{~d} t}=\sum_{n^{\prime}}\left[w_{n n^{\prime}}(\mathbf{c}, t) \rho_{n^{\prime}}(t)-w_{n^{\prime} n}(\mathbf{c}, t) \rho_{n}(t)\right] .
\end{gathered}
$$

Here, the right hand side of the upper equation corresponds to $\mathbf{F}(\mathbf{c}, S, t)$ of fig. 4.1. Since the stochastic part $g_{k}$ of eq. (2.24) can only be in one state at a time, it can be rewritten as:

$$
g_{k}(\mathbf{c}, S(t), t)=\sum_{n} \delta_{S(t), S_{n}} g_{k}^{(n)}(\mathbf{x}, t) .
$$

The above sum over Kronecker deltas will always reduce to only one summand. Hence, during periods where no change of state $S$ occurs the system's dynamics are fully governed by the functions $f_{k}$ and the corresponding $g_{k}^{(n)}$ of the state $S$. 

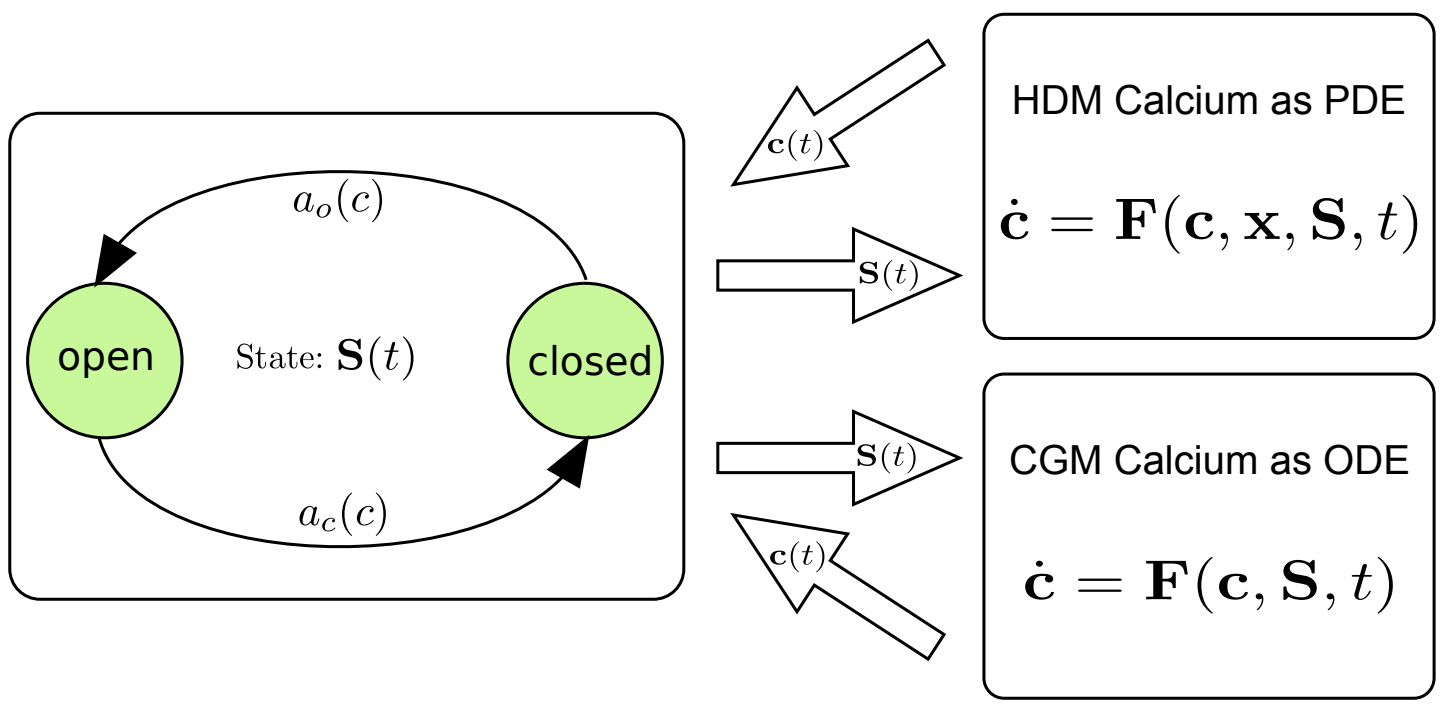

Fig. 4.1: Interdependence of stochastic and deterministic part. The integration of the deterministic part depends on the state of the Markov process. On the other hand, the transition rates of the Markov process depend on the concentrations described by the deterministic part.

The above equations already give a hint at how to sample trajectories from such a system. Given a composed initial state $\left\{\mathbf{c}_{o}, S_{j}\right\}$ at time $t_{0}$, one can integrate the system for given $g_{k}^{(j)}$ up to a time $t_{1}$ as one would integrate a normal deterministic system. However, as soon as the system state changes, integration continues with a different $g_{k}^{(n)}$. At this point the dwell time distribution from section 2.2 comes into play: since the solution $\mathbf{c}(t)$ for $t \in\left[t_{0}, t_{1}\right]$ is known, one can integrate the left hand side of eq. (2.15) for that interval. Given a randomly chosen $\chi$ at $t_{0}$ one continues to integrate the system until the right hand side of eq. (2.15) exceeds the left hand side. During the integration step $t_{i}$ to $t_{i+1}$, at which $\chi$ gets exceeded some jump of state $S$ has occurred. If that happened, one solves eq. (2.15) for the jump time $t_{\text {jump }} \in\left[t_{i}, t_{i+1}\right]$. Then, one "rolls back" the deterministic part to $t_{\text {jump }}$, calculates the new state $S_{m}$ where the systems jumps to according to eq. (2.18), and picks a new random $\chi$. Afterwards, one simply continues integration with the new function $g_{k}^{(m)}$. Algorithm 1 gives a pseudo code example of the above method, which closely reflects the real code that was used.

In practice, some optimizations can be applied:

- If the system consists of many identical indistinguishable units where each unit has its own internal state, one can switch to an "occupation number" formalism. The combined system state is then defined by a state vector $\mathbf{S}$ where each component $i$ of $\mathbf{S}$ denotes the number of particles that are in state $i$. I.e. $S_{i} \in[0, N]$ and 


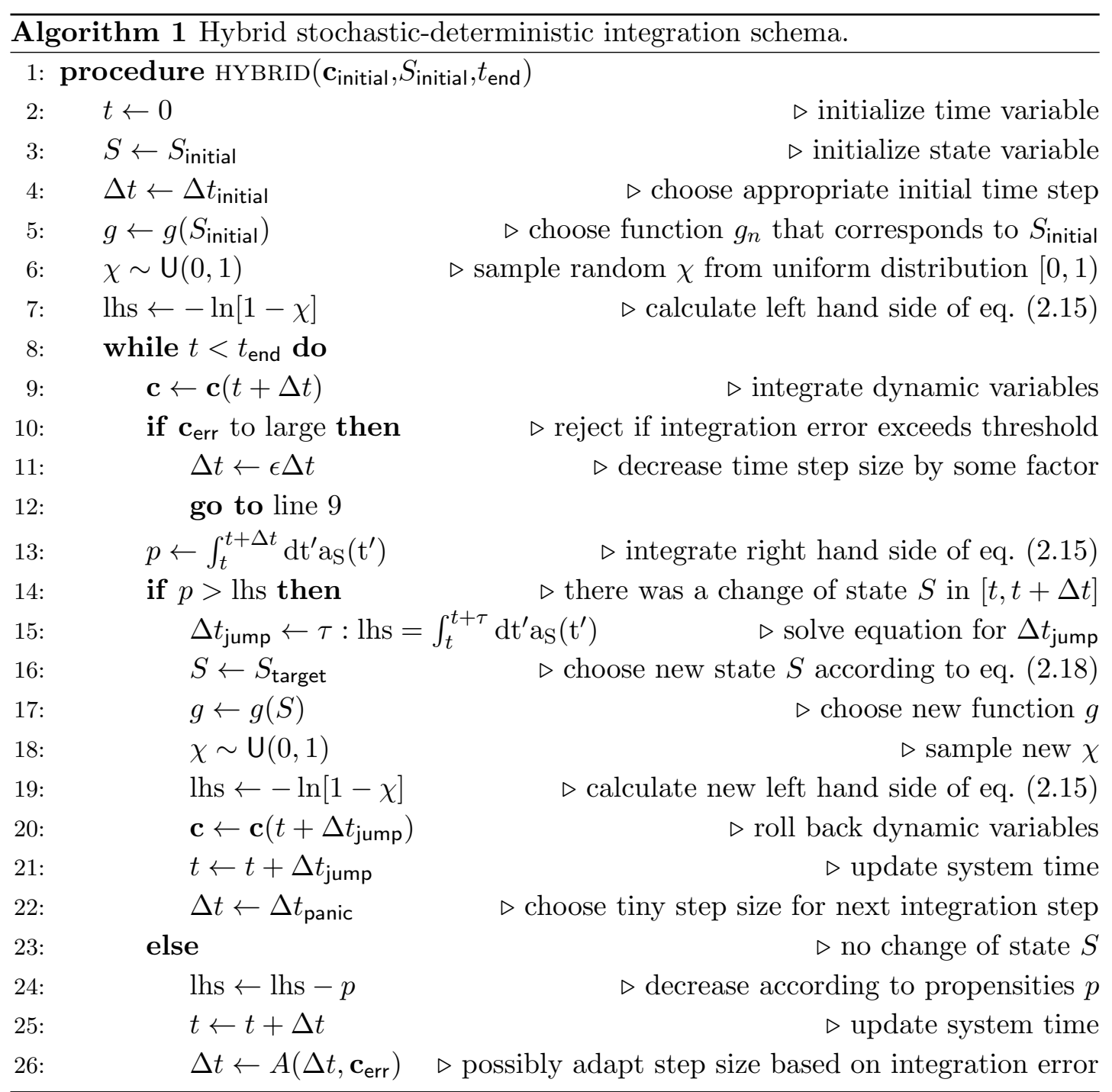


$\sum_{i} S_{i}=N$ where $N$ is the number of particles in the system. For details and an application see section 3.4.

- If there are multiple states $S$ with identical function $g$ one only needs to "roll back" the dynamic variables if a jump occurred that changed the respective $g$. Otherwise one just takes the different $S$ in the integration of the right hand side of eq. (2.15). This performance optimization is crucial in the context of the DYK model because of its vast amount of jumps between different occupation numbers (see section 3.4). Effectively, only a tiny fraction of those jumps correspond to a channel opening or closing, while their majority reflects multi step reactivation or equilibrium fluctuations between several closed states.

- Often an adaptive time step is used for the integration of the dynamic system. If the time steps $\Delta t$ are small enough, one can approximate the right hand side of eq. (2.15) as $\Delta t a_{S}(t)$.

\subsection{Local spatial refinement and adaptive step size}

In this work, the time steps $\Delta t(h)$ from from section 2.5.2 were chosen adaptively. If a channel opens or closes, the time step for the next integration step is set to some tiny value $\Delta t_{\text {panic }}$ to account for the rapid change of boundary conditions (see algorithm 1 line 22). This avoids frequent rejections (line 10) of the expensive integration step (line 9). If no channel opens or closes, the time step size will be adopted according to algorithm 2. Parameters of the adaptive time stepping which were used throughout this work are given in table 4.1 .

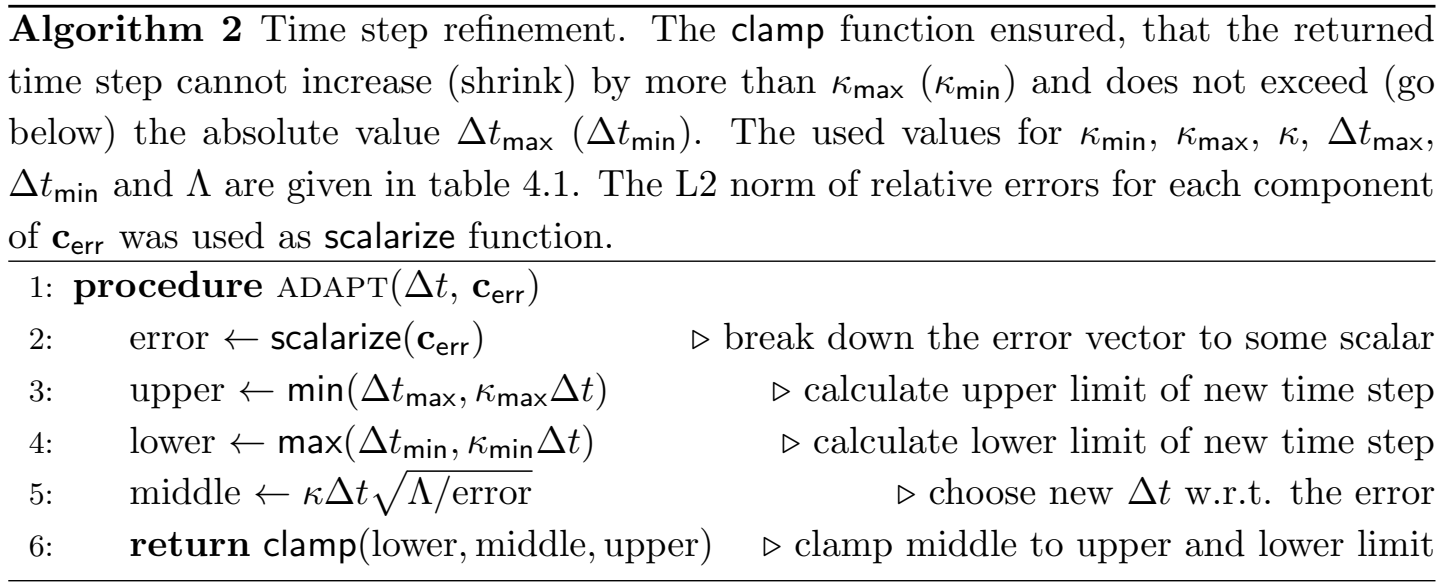



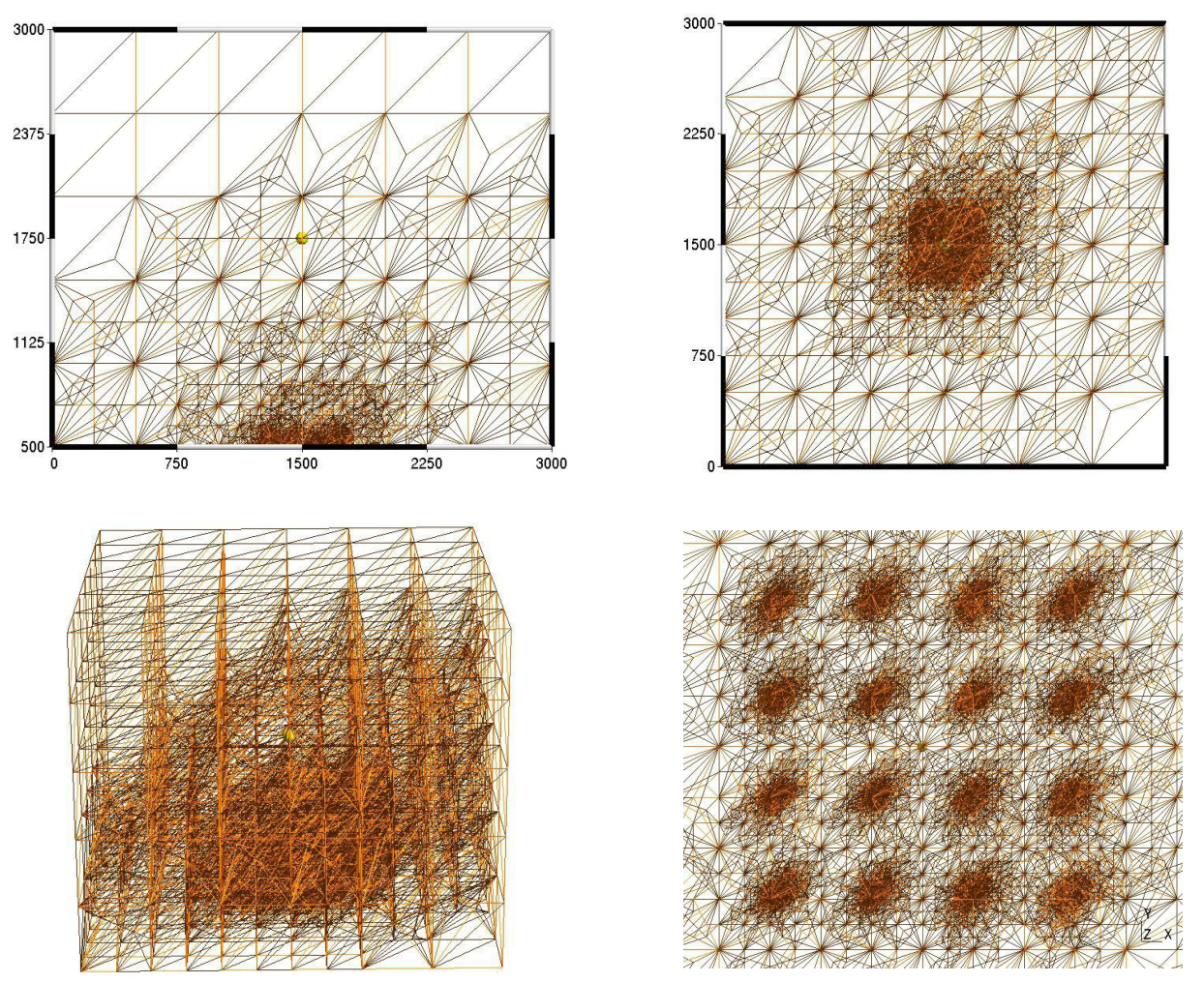

Fig. 4.2: Level 7 grid that was used for the FEM calculations in section 5.3. From top left to bottom right: projection along $\mathrm{x}$-axis, projection along $\mathrm{z}$-axis, perspective view along $\mathrm{x}$-axis, and zoomed projection along z-axis.

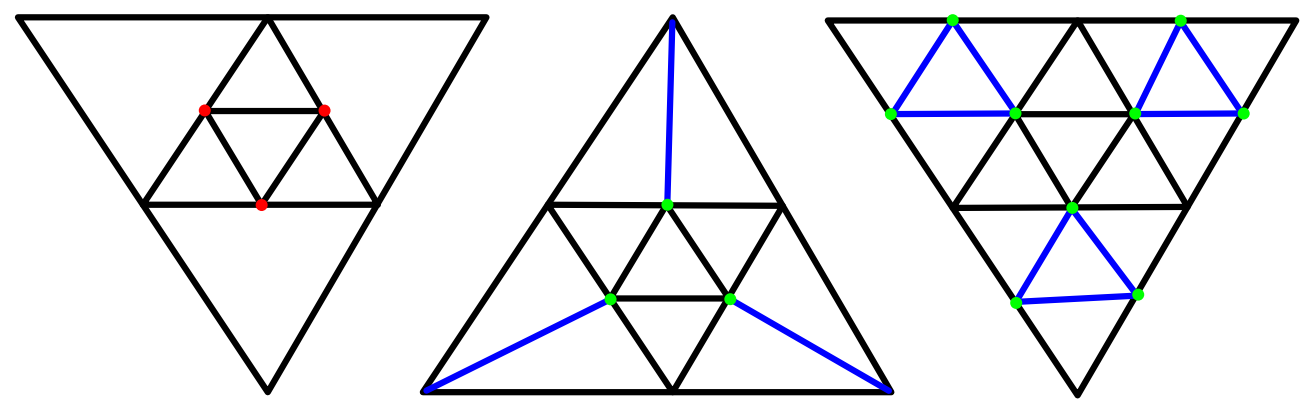

Fig. 4.3: Left: Local non conforming refinement of the center element. To keep a continuous solution one has to put constraints on the three new degrees of freedom (red dots). Middle: Local conforming refinement. Right: Global refinement introducing 9 additional degrees of freedom. 


\begin{tabular}{l|c|l}
\hline maximum relative decrease & $\kappa_{\min }$ & 0.1 \\
maximum relative increase & $\kappa_{\max }$ & 2 \\
increase factor & $\kappa$ & 0.95 \\
minimum time step & $\Delta t_{\min }$ & $0.1 \mathrm{~ns}$ \\
maximum time step & $\Delta t_{\max }$ & $0.2 \mathrm{~s}$ \\
error threshold & $\Lambda$ & $0.1 \%$ \\
transition collapse & $\Delta t_{\text {panic }}$ & $10 \mathrm{~ns}$ \\
\hline
\end{tabular}

Tab. 4.1: Parameters used for adaptive time stepping in algorithm 1 and algorithm 2 .

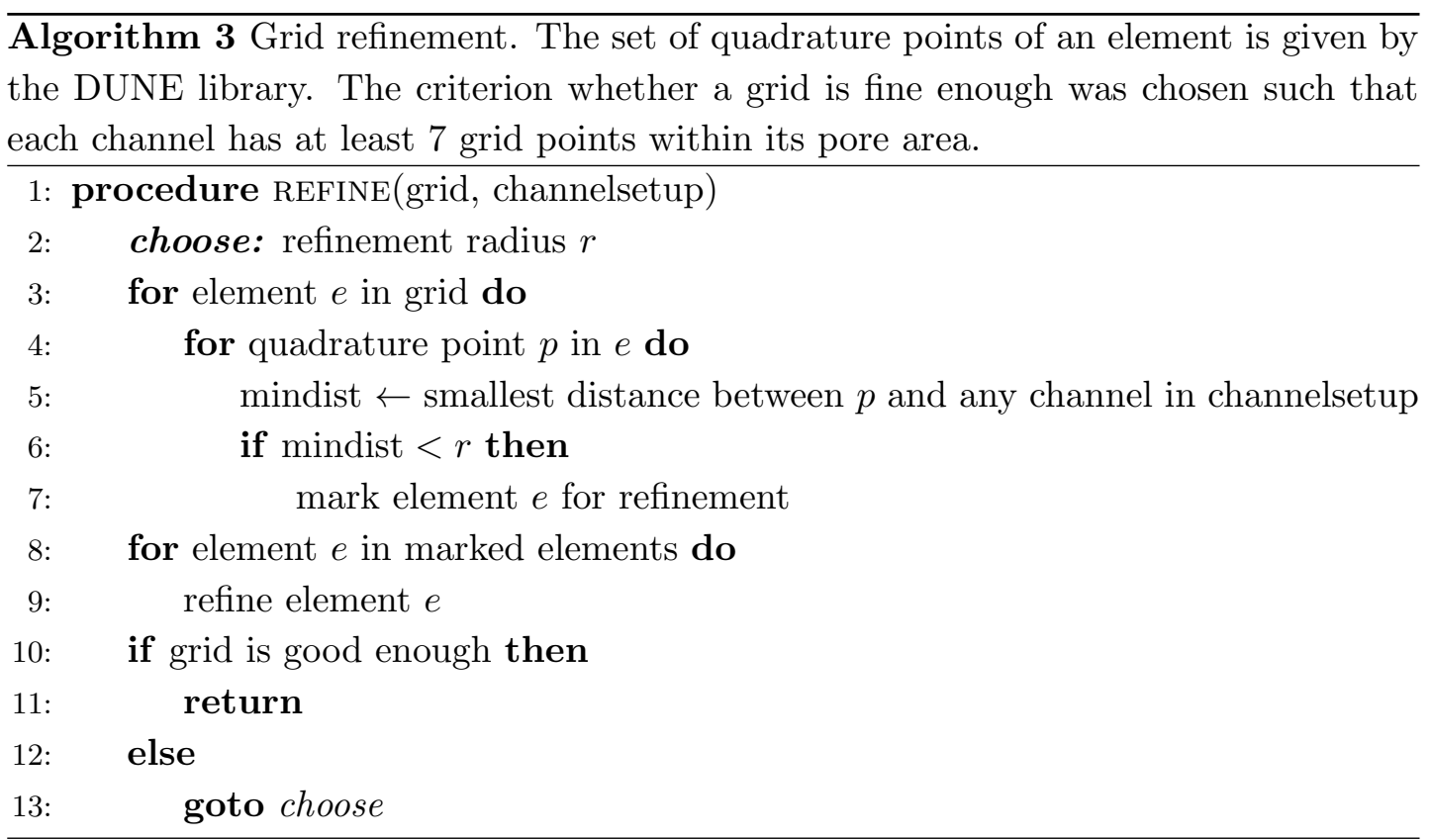

In contrast to the dynamic discretization of time, the grid used for the results presented in section 5.3 is static. While the used C++ framework (DUNE, [Bas+08b; Bas+08a]) provides functionality for time dependent adaptive grids, this adaptivity introduces a large amount of computational overhead per time step. One possible improvement may be to couple the grid refinement to the channel states, i.e. locally refine the grid at open channel pores where large concentration gradients exist while coarse graining in the area of closed clusters. However, this approach was not followed in this work. In this work, the grid consisted of simplices, i.e. tetrahedrons and is conforming, which means that one surface of any tetrahedron exactly touches one neighboring tetrahedron with exception of boundary facets (which do not have any 
neighbor, see fig. 4.3). The refinement strategy of the grid is denoted in algorithm 3 . In order to keep the conforming property of the grid, each refinement of a tetrahedron will always result in four child tetrahedrons. The resulted grid is depicted in fig. 4.2.

\subsection{Event definitions}

The stochastic gating of the DYK model which is used throughout this work (see section 3.4) provides the full state of each channel at each point in time by keeping track of every single transition of all subunits of all simulated channels. ${ }^{1}$ Because of feedback effects, coupling, and clustering of channels these transitions are highly correlated. Hence, to investigate the collective behavior of an $\mathrm{IP}_{3} \mathrm{R}$ cluster, it is essential to group synchronous channel openings in collective events (CEs). A collective event is defined by its start- and end-point in time, during which the cluster of channels is active. Usually the start point falls together with the first channel opening, and the endpoint follows the last channel closing. While a separation into a set of collective events by hand often seems straight forward, several choices have to be made when providing an automated definition, which is required for reproducibility and analysis of large data sets.

Another important consideration arises from the experimental data with which one wants to compare the simulated results. Comparison with fluorescence data may have other requirements on the $\mathrm{CE}$ definition than data originating from patch clamp experiments. For example, fast single channel dynamics often get averaged out of calcium fluorescence measurements [SP05]. In that case, applying an event definition which solely relies on the number of open channels is a bad choice because it does not account for the the dye buffer kinetics. On the other hand, for membrane current recordings (see e.g. [MF97]), the simulated number of open channels may actually provide the best match, since feedback effects like CICR and channel re-openings due to residual $\mathrm{Ca}^{2+}$ can be avoided. ${ }^{2}$

Given a proper definition, one can analyze a variety of different event properties and their distributions. Some of which are:

- Event duration. Because the duration is usually accessible from experiments,

\footnotetext{
${ }^{1}$ For example in the simulations presented in section 5.3 for an $\mathrm{IP}_{3}$ concentration of $70 \mathrm{nM}$ a single channel is subject to about 50 transitions per second yielding a total amount of $16 \times 50 \mathrm{~s}^{-1} \times 3000 \mathrm{~s}=$ 2400000 transitions. In the later multi cluster simulations from section 6.2 this number rises up to $64 \times 16 \times 50 \mathrm{~s}^{-1} \times 6000 \mathrm{~s}=307200000$ transitions.

${ }^{2}$ In patch clamp recordings, the carried charge may not be $\mathrm{Ca}^{2+}$ but some other ion, or the experimental setup may continuously remove the $\mathrm{Ca}^{2+}$ which passed through the channels. This yields steady state statistics of channel gating.
} 
this is probably the most widely analyzed property. While simple, it may already provide many insights into the underlying cluster dynamics. Further, correlations between successive events allow to fit even more model parameters to experiments. For example, one can draw conclusion about the gating schema if the system occasionally transitions between a bursting and non bursting state [Ion+07, Fig. 1].

- Peak number of open channels. This quantal property can be measured in patch clamp [MF97; Tau+09], and since recent advances also in fluorescence imaging experiments [SP09; Bru+10; DSP12; Ull+12; DP13]. The distribution of the number of open channels gives interesting insights into the overall "availability" of channels of a cluster. For example, a large fraction of channels may be inhibited or deactivated almost permanently.

- Accumulated efflux. Integrating the efflux of $\mathrm{Ca}^{2+}$ through all open channels can provide hints at possible depletion effects of the ER or SR [RLS11].

- States of gating model at start and end. The state of the channels at the beginning of a collective event may dictate the course of the whole event. If more channels are available, the event duration may be longer. On the other hand, if channels are in some long enduring inhibited state at the end of an event, the time to the next event can be prolonged.

- Inter event interval. Like the event duration, the time between successive events is usually accessible from experiments and can therefore be used to fit model parameters [MP01].

\subsection{Synchronization}

To obtain information such as the inter wave interval or global oscillation frequencies, it is important to have a good measure of synchronization between different clusters. In this work, two different techniques will be applied. First, synchronization between CE will be investigated with a symmetric version of the method called event synchronization (ES) introduced by Quiroga et al. [QKG02]. Second, the complex coherence function (CCF) [Pri81; Mar87; CM13] will be used to measure coherence between calcium concentrations originating from different clusters.

\subsubsection{Event Synchronization}

Calculating the event synchronization (ES) is a two step process. An event series for a cluster $n(m)$ is defined by the start times $t_{n}^{\mu}$ and $t_{m}^{\nu}$ of the events, where $\mu$ and $\nu$ 
denote the indices of the events. With this notation, the time between the $\mu$-th event in cluster $n$ and the $\nu$-th event in cluster $m$ is given as $d_{n m}^{\mu, \nu}=t_{n}^{\mu}-t_{m}^{\nu}$. First one calculates the dynamical delay $\tau_{n m}^{\mu \nu}$ between the two event series as follows:

$$
\tau_{n m}^{\mu \nu}=\frac{1}{2} \min \left[d_{n n}^{\mu-1, \mu}, d_{n n}^{\mu, \mu+1}, d_{m m}^{\nu-1, \nu}, d_{m m}^{\nu, \nu+1}\right] .
$$

The synchronization matrix between two event series is then given as:

$$
J_{n m}^{\mu \nu}= \begin{cases}1, & \text { if } \quad 0<\left|d_{n m}^{\mu, \nu}\right| \leq \min \left[\tau_{n m}^{\mu \nu}, \tau_{\max }\right] \\ 0, & \text { else, }\end{cases}
$$

where $\tau_{\max }$ denotes a maximum delay between events. In this work $\tau_{\max }$ is chosen as half of the average inter-event distance of the respective simulation [QKG02]. The absolute value $\left|d_{n m}^{\mu, \nu}\right|$ in eq. (4.3) makes the ES measure symmetric, i.e. the $\mu$-th event will count as synchronized with the $\nu$-th event, irrespective of whether $\mu$ precedes or succeeds $\nu$.

\subsubsection{Sample coherence function}

The complex coherence function (CCF) $C_{i j}$ of two signals $c_{i}$ and $c_{j}$ is defined as fraction of the cross spectral density (CSD) $P_{i j}$ and the product of the two power spectral density (PSD) $P_{i i}$ and $P_{j j}$ of the signals [CK91; CKN73]:

$$
C_{i j}(\omega)=\frac{P_{i j}(\omega)}{\sqrt{P_{i i}(\omega) P_{j j}(\omega)}}
$$

The cross spectral density matrix $P_{i j}$ is given as follows:

$$
P_{i j}(\omega)=\mathcal{F}\left(c_{i}\right)^{*} \times \mathcal{F}\left(c_{j}\right) .
$$

Here $\mathcal{F}(c)$ and ${ }^{*}$ denote the Fourier transform of $c$ and complex conjugation, respectively. Note that in general all but the diagonal entries of $C_{i j}$ will be complex. Further, the matrix $C_{i j}$ is Hermitian. The cross spectral density is connected with the cross correlation via the Wiener Khinchin theorem, i.e. the convolution of the cross correlation in time becomes a product in Fourier space.

The advantage of the CCF is that is measures similarity between two signals irrespective of a possible phase lag $\Delta \phi_{i j}$ between them. Further, the phase lag between the signals is given by the angle of $C_{i j}$, i.e. $\Delta \phi_{i j}=\arg \left(C_{i j}\right)$.

If the coherence for low frequencies $\omega$ (period in the range of $5 \mathrm{~s}$ to $60 \mathrm{~s}$ ) is of interest, a high resolution in the frequency domain (e.g. $0.01 \mathrm{~Hz}$ ) will be required. 
This premises Fourier transformations of time intervals larger than $100 \mathrm{~s}$. Further, good estimates of the CCF can be obtained with an extension of Welch's method [Wel67] as done in [Smi07]. In Welch's method, the Fourier transform is calculated for several overlapping windows of the data. The power spectrum is then approximated by taking the average of the Fourier transform w.r.t. the windows. Therefore, $S$ sample sequences $c_{i}^{(0)}, \ldots, c_{i}^{(S-1)}$ of the data are created, where each sequence is discrete in time and given as $c_{i}^{(n)}=c_{i}^{(n, 0)}, \ldots, c_{i}^{(n, L-1)}$ where $c_{i}^{(n, k)}=h(k) c_{i}(\Delta t(k+n D))$. Thereby $L \Delta t$ denotes the window size and $D$ determines the overlap of the windows. The $h$ defines a windowing function, which gets multiplied on the data $c_{i}$ before applying the Fourier transform. Throughout this work, $h$ will be chosen as what is called the "Hanning" window which is defined as:

$$
h(k)=0.5-0.5 \cos \left[\frac{2 \pi k}{L-1}\right] \quad 0 \leq k \leq L-1 .
$$

With this, the sample coherence function $(\mathrm{SCF}) \widehat{C}_{i j}$ is given as follows:

$$
\widehat{C}_{i j}(\omega)=\frac{\left\langle\mathcal{F}\left(c_{i}^{(n)}\right)^{*} \times \mathcal{F}\left(c_{j}^{(n)}\right)\right\rangle_{S}}{\sqrt{\left\langle\left|\mathcal{F}\left(c_{i}^{(n)}\right)\right|^{2}\right\rangle_{S}\left\langle\left|\mathcal{F}\left(c_{j}^{(n)}\right)\right|^{2}\right\rangle_{S}}} .
$$

Here, the angle brackets $\langle\ldots\rangle_{S}$ denote the average over the $S$ overlapping windows. Further, the continuous Fourier transform from eq. (4.5) gets replaced by the discrete Fourier transform.

Note, that in the enumerator of eq. (4.7) the average value of a complex value is calculated. The magnitude of the CCF is supposed to range between 0 and 1 , denoting completely incoherent, and identical signals, respectively. Obviously one cannot commute the magnitude operation with the average in the enumerator. As discussed in [CK91] this leads to different averaging strategies. In many cases the magnitude squared coherence (MSC) is used instead of the CCF. Its estimate directly follows from eq. (4.7) with:

$$
\overline{\operatorname{MSC}}_{i j}(\omega)=\left|\widehat{C}_{i j}\right|^{2}=\frac{\left|\left\langle\mathcal{F}\left(c_{i}^{(n)}\right)^{*} \times \mathcal{F}\left(c_{j}^{(n)}\right)\right\rangle_{S}\right|^{2}}{\left\langle\left|\mathcal{F}\left(c_{i}^{(n)}\right)\right|^{2}\right\rangle_{S}\left\langle\left|\mathcal{F}\left(c_{j}^{(n)}\right)\right|^{2}\right\rangle_{S}} .
$$

While calculation of the SCF or sampled MSC is straight forward with the above equations, the average in the enumerator of eqs. (4.7) and (4.8) results in a major drawback: The estimate of coherence, i.e. the magnitude, will always be biased by an amount $\Delta$ towards higher values! For the detailed derivations of an estimation of the 
bias and its variance, the interested reader is referred to [CKN73]. The bias of eq. (4.8) is given as [CKN73]:

$$
\Delta=E[\overline{\mathrm{MSC}}, S]-\mathrm{MSC}=\frac{1}{S}+\frac{S-1}{S+1} \mathrm{MSC}_{2} F_{1}[1,1 ; S+2 ; \mathrm{MSC}]-\mathrm{MSC} .
$$

Here, ${ }_{2} F_{1}$ is the (Gauss) hypergeometric function, $E$ denotes the expectation value, $S$ again denotes the number of windows of the data series, and the unknown exact MSC is denoted without the over bar. One can obtain an unbiased estimate by solving the following equation for MSC numerically:

$$
0=\frac{1}{S}+\frac{S-1}{S+1} \mathrm{MSC}_{2} F_{1}[1,1 ; S+2 ; \mathrm{MSC}]-\overline{\mathrm{MSC}} .
$$

If one is interested in an average synchronization of many different signals $c_{0}, \ldots, c_{N}$, one can average the SCF or MSC over all pairs of signals. Again, the average of the SCF is not as straightforward as it seems, since simply averaging the complex valued $\widehat{C}_{i j}$ over pairs of $i$ and $j$ yields unexpected results. First, the phases lags between the signal pairs $\Delta \phi_{i j}$ are distributed symmetrically around zero. Hence, they will cancel out in the averaging process. ${ }^{3}$ Second, while all pairs might have a absolute coherence of 1 for a certain frequency, averaging the complex values might yield an average coherence less than unity. ${ }^{4}$

To avoid the above problems, one can calculate the average of the absolute phase lag $\left\langle\left|\arg \left[\widehat{C}_{i j}\right]\right|\right\rangle_{i \neq j}$ and recompose it with the MSC into a single complex valued function:

$$
\widehat{C}=\left\langle\mathrm{MSC}_{i j}\right\rangle_{i \neq j} \exp \left[\mathrm{i}\left\langle\left|\arg \left[\widehat{C}_{i j}\right]\right|\right\rangle_{i \neq j}\right]
$$

To test the above method sinusoidal signals with different phase lags were created:

$$
c_{i}(t)=\sin \left[\omega_{0} t+\Delta \omega_{i}\right]+\alpha \xi_{i}(t)
$$

with

$$
\Delta \omega_{i} \sim[0, \pi] \text { and }\left\langle\xi_{i}(t) \xi_{j}\left(t^{\prime}\right)\right\rangle=\delta_{i j} \delta\left(t-t^{\prime}\right) .
$$

Further, to check the sensitivity of the SCF w.r.t. to noise, Gaussian white noise $\xi(t)$ with intensity $\alpha$ was added on top of the sinusoidals. Figure 4.4 A shows the SCF as given in eq. (4.7) for two sinusoidal signals with fixed phase lag for different noise intensities. In fig. $4.4 \mathrm{~B}$ eq. (4.11) has been applied to a set of 30 noisy sinusoidals.

\footnotetext{
${ }^{3}$ The phase lag of $c_{i}$ w.r.t. $c_{j}$ is the negative phase lag of $c_{j}$ w.r.t. $c_{i}$, i.e. $C_{i j}=C_{j i}^{*}$ or $\Delta \phi_{i j}=-\Delta \phi_{j i}$.

${ }^{4}$ Following from the triangle inequality: $\left|z_{1}+z_{2}\right| \leq\left|z_{1}\right|+\left|z_{2}\right| \forall z_{1}, z_{2} \in \mathbb{C}$.
} 

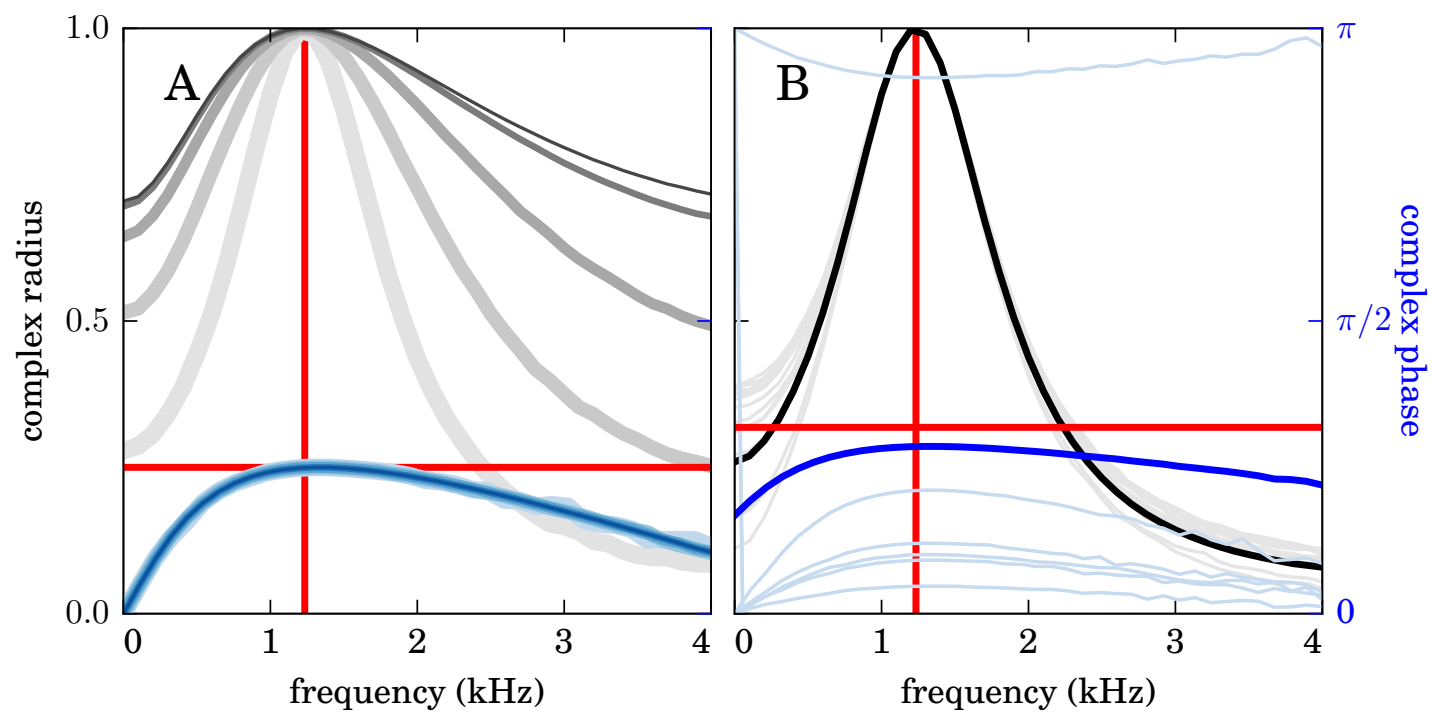

Fig. 4.4: Sample coherence function for noisy sine waves. Black: absolute coherence (left axis), Blue: phase lag (right axis). The red lines denote the main frequency $\omega_{0}$ and average phase lags for the signals. (A) SCF for two signals with different noise intensities (amplitudes $\alpha$ increase with line width: $0.01,0.02,0.05,0.1,0.2$ ). (B) Averaged sample coherence function for 30 noisy sine waves according to eq. (4.11). The phase lag of the signals was uniformly distributed in $[0, \pi]$ resulting in an average absolute phase lag of $1 \approx 0.318 \pi$. The opaque lines denote the SCF for randomly chosen individual pairs. Noise amplitude: $\alpha=0.2$.

With the above definition, a full coherence $\left|\widehat{C}\left(\omega_{0}\right)\right|=1$ and an average absolute phase lag $\arg \left[\widehat{C}\left(\omega_{0}\right)\right]=1$ is observed at $\omega=\omega_{0}$, which corresponds to the expected outcome. ${ }^{5}$ For both figures, $5 \times 10^{4}$ data points with a sampling frequency of $10^{3} \mathrm{~Hz}$ were used. Other parameters: $\omega_{0}=1234 \mathrm{~Hz}$, overlap $D=0.5$, window size $L=100$. Increasing the window size results in more noisy data points of the SCF, since a larger window size decreases the number of sampling windows $S$.

\footnotetext{
${ }^{5}$ The distribution of phase lags $\Delta \phi_{i j}$ has a triangular shape and follows from the uniform distribution of $\Delta \omega_{i}$ as

$$
\rho\left(\Delta \phi_{i j}\right)=\left\{\begin{array}{ll}
-\frac{1}{\pi^{2}}\left|\Delta \phi_{i j}\right|+\frac{1}{\pi}, & \Delta \phi_{i j} \in[-\pi, \pi] \\
0, & \text { else }
\end{array} .\right.
$$
}




\section{Chapter 5}

\section{Calcium as a concentration field}

\subsection{Model description}

In this chapter the interaction of $\mathrm{Ca}^{2+}$ with buffers and the diffusion of $\mathrm{Ca}^{2+}$ and buffers are treated as a system of coupled reaction-diffusion equations:

$$
\begin{aligned}
& \frac{\partial c}{\partial t}=D_{c} \nabla^{2} c+\sum_{i} b_{i} k_{i}^{-}-c k_{i}^{+}\left(B_{i}-b_{i}\right) \\
& \frac{\partial b_{i}}{\partial t}=D_{i} \nabla^{2} b_{i}-b_{i} k_{i}^{-}+c k_{i}^{+}\left(B_{i}-b_{i}\right) .
\end{aligned}
$$

Here $c$ and $b_{i}$ will denote the free cytosolic $\left[\mathrm{Ca}^{2+}\right]$ and bound buffer concentration of different species $i$, respectively. The indices $c$ and $i$ will also be used to distinguish between different other species-specific parameters, i.e. the diffusion coefficients $\left(D_{c}\right.$ and $\left.D_{i}\right)$, the total buffer concentration $\left(B_{i}\right)$, the bound buffer concentration $\left(b_{i}\right)$ and the binding $\left(k_{i}^{+}\right)$and unbinding $\left(k_{i}^{-}\right)$rates of the buffers.

The bound buffer concentration for resting conditions $b_{i, 0}$ follows directly from eq. (5.2) when setting the 1.h.s. and the spatial derivative to zero and the calcium concentration to $c_{0}$ :

$$
b_{i, 0}=\frac{c_{0} k_{i}^{+} B_{i}}{c_{0} k_{i}^{+}-k_{i}^{-}}
$$

Reaction and diffusion takes place in some domain $\Omega$ with boundary $\partial \Omega$. In principle the boundary can be one of two different kinds:

- A boundary representing the membrane of an organelle or the plasma membrane will be denoted as $\partial \Omega_{M}$. This boundary imposes special restrictions on the cal- 
cium ion flux: it might be impermeable or contain proteins that actively transport calcium. Further, the membrane is assumed to be impermeable for buffers.

- A "virtual" boundary $\partial \Omega_{V}$ that solely serves the purpose of restricting the volume for which the reaction diffusion equations need to be solved.

To complete the description of the concentration fields given in eqs. (5.1) and (5.2) one also needs to impose boundary conditions (BCs) on these boundaries. In general no flux Neumann BCs for all buffers on the whole domain boundary $\partial \Omega$ are used:

$$
D_{i} \vec{n} \vec{\nabla} b_{i}=0, \text { at } \partial \Omega \text {. }
$$

Here, $\vec{n}$ denotes the outer normal vector of the boundary. For the free calcium concentration the boundary condition on the virtual boundary is again a no flux Neumann BC:

$$
D_{c} \vec{n} \vec{\nabla} c=0, \text { at } \partial \Omega_{V}
$$

However, the flux through the membrane boundaries is defined by a function $J(\vec{r}, t)$ :

$$
D_{c} \vec{n} \vec{\nabla} c=J(\vec{r}, t), \text { at } \partial \Omega_{M} .
$$

While the definition of $J$ generally depends on the system of interest, this work follows what was used in $[\mathrm{Ruc}+15]$ :

$$
J=P_{\mathrm{c}} S(\vec{r}, t)(E-c)-P_{\mathrm{p}} \frac{c^{2}}{K_{\mathrm{d}}^{2}+c^{2}}+P_{\mathrm{l}}(E-c) .
$$

Here, $\vec{r}$ represents the spatial coordinate with $z=0$ denoting the ER membrane. The three terms in the equation model channel efflux, SERCA pump current and leak current, respectively.

First, the channel efflux is proportional to the $\left[\mathrm{Ca}^{2+}\right]$ within the ER depicted as $E$, and it vanishes whenever a channel is closed, i.e. it is controlled by a factor depending on the channels' states $S(\vec{r}, t)$ :

$$
S(\vec{r}, t)=\left\{\begin{array}{cc}
1, & \text { if there is an open channel } k \\
& \text { with }\left|\vec{r}-\vec{X}_{k}\right|<R_{\mathrm{c}}, \\
0, & \text { otherwise. }
\end{array}\right.
$$

Here, the channel pore radius is given as $R_{c}$, and $k$ denotes the index of a channel. The electrical channel current $I_{c}$ can be calculated as the area integral:

$$
I_{c}(t)=\int_{\text {pore }} 2 F P_{c}(E-c(\vec{r}, t)) d \vec{A},
$$


where $F=e N_{a}$ is the Faraday constant. Equation (5.9) can be approximated as $I_{c} \approx 2 F R_{c}^{2} \pi P_{c} E$. This allows to chose $P_{c}$ to obtain a channel current of $I_{c}=0.07 \mathrm{pA}$ as was used in [Ull+12; Bru+10; SP05; VFM10]. One downside of eqs. (5.7)-(5.9) is the dependency of the $\left[\mathrm{Ca}^{2+}\right]$ peak amplitude at the channel pore of an open channel on the channel pore radius $R_{c}$. Smaller pore radii will in general lead to sharper $\mathrm{Ca}^{2+}$ profiles but also significantly increase computation time, because they require a much higher grid resolution in the channel area. For the chosen value $P_{c}=4.58 \times 10^{6} \mathrm{~nm} / \mathrm{s}$ and $R_{c}=6 \mathrm{~nm}$ the peak amplitude is around $70 \mu \mathrm{M}$ to $80 \mu \mathrm{M}$. Simulations with more realistic smaller $R_{c}=2.5 \mathrm{~nm}$ reached peak values of $150 \mu \mathrm{M}$ to $200 \mu \mathrm{M}$. Therefore, the DYK channel model is not fully coupled to the peak $\left[\mathrm{Ca}^{2+}\right]$ which results from the above equations. Instead, in the case of an open channel, the $\left[\mathrm{Ca}^{2+}\right]$ which gets fed to the DYK model is artificially clamped to a constant value of $150 \mu \mathrm{M}$.

The second term in eq. (5.7) models SERCA pumps. As shown in [Lyt+92], isoforms of SERCA pumps get activated by $\mathrm{Ca}^{2+}$ in a cooperative manner with a Hill coefficient of 2. ${ }^{1}$ According to [Lyt+92, Fig. 4] the dissociation coefficient of SERCA ranges from $0.3 \mu \mathrm{M}$ to $1 \mu \mathrm{M}$. The maximum $\mathrm{Ca}^{2+}$ uptake of SERCA was estimated as $5 \mu \mathrm{M} / \mathrm{s}$ to $16 \mu \mathrm{M} / \mathrm{s}$ depending on the considered isoform of SERCA [IKL88; Sch89; Lyt+92; Fal04]. The estimated pump current of $5 \mu \mathrm{M} / \mathrm{s}$ to $16 \mu \mathrm{M} / \mathrm{s}$ is based on a concentration of pumps within the cell, hence to obtain a boundary flux as required by eq. (5.7) one needs to project this volume average onto the membrane by multiplying it with the extent of the domain $d_{z}{ }^{2}$

The last term in eq. (5.7) is required because the SERCA model does not depend on lumenal $\left[\mathrm{Ca}^{2+}\right]$ : even at low $\left[\mathrm{Ca}^{2+}\right]$, pumps would constantly be active and in the long-run the ER would overload. In [Fav+96] Favre et. al. argue, that $\mathrm{Ca}^{2+}$ uptake into the ER saturates due to a negative feedback of SERCA on lumenal $\left[\mathrm{Ca}^{2+}\right]$. However, for the sake of simplicity, in this work saturation is enforced via a leak current which

\footnotetext{
${ }^{1}$ SERCA also gets activated by ATP with a Hill coefficient of 1 [Lyt+92]. The values provided in this work all assume saturated activation via ATP which represent the upper limit of transport capabilities.

${ }^{2}$ While it is a trivial calculation, the units of the variables in eq. (5.7) are not evident on first sight. In the following [...] denotes the dimension of the operand, $L$ and $T$ are space and time, respectively:
}

$$
\begin{aligned}
{[D \vec{n} \vec{\nabla} c]=[J] } & =\frac{L^{2}}{T} \times \frac{1}{L} \times \frac{1}{L^{3}}=\frac{1}{T L^{2}} \\
& \Longrightarrow\left[P_{c}\right]=\frac{1}{T L^{2}} \times L^{3}=\frac{L}{T} \\
& \Longrightarrow\left[P_{p}\right]=\frac{1}{T L^{2}} \\
& \Longrightarrow\left[P_{l}\right]=\left[P_{c}\right]=\frac{L}{T} .
\end{aligned}
$$




\begin{tabular}{l|c|c|c|c} 
Buffer & $D_{b}$ in $\mu \mathrm{m}^{2} / \mathrm{s}$ & $k^{+}$in $1 / \mu \mathrm{Ms}$ & $k^{-}$in $1 / \mathrm{s}$ & $K$ in $\mu \mathrm{M}$ \\
\hline stationary endogenous & 0.01 & 150 & 300 & 2 \\
\hline BAPTA & 95 & 600 & 100 & 0.17 \\
EGTA & 113 & 1.5 & 0.3 & 0.2
\end{tabular}

Tab. 5.1: Buffer diffusion coefficients and binding parameters. In the following simulations, a single stationary buffer with the parameters from the first row was used. Its diffusion coefficient is chosen slightly larger than zero for numerical reasons only. The values for BAPTA and EGTA originate from $[\mathrm{KS} 10$, p. 312] and [Smi+01]. A concise overview about different dye buffers can be found in $[\mathrm{Par}+08]$.

drives the system to its resting concentration $c_{0}$. This can be achieved by choosing $P_{l}$ as:

$$
P_{1}=\frac{P_{\mathrm{p}} c_{0}^{2}}{\left(E-c_{0}\right)\left(K_{\mathrm{d}}^{2}+c_{0}^{2}\right)} .
$$

\subsection{Simulations}

The model introduced in section 5.1 was simulated for various different $\mathrm{IP}_{3}$ concentrations. Additional model parameters are given in tables 3.1, 4.1, 5.1 and 5.2. The following subsections will cover different aspects and results of those simulations.

Because reinventing the wheel is in general a bad idea when it comes to advanced numerical methods, this work makes extensive use of the Dune $\mathrm{C}++$ framework for spatial discretization and parallelization to multiple CPUs [Bas+08b; Bas+08a] and assembly of the FEM matrices (Dune PDELab, [BHM10]). The resulting equation systems are solved with Dune's Iterative Solver Template Library (ISTL) [BB07; BB08]. In fact, much of the work in this thesis is based on a core implementation by $\mathrm{N}$. Chamakuri which has been used in e.g. [Rud+07], but was significantly extended and modularized. The resulting large scale system of sparsely coupled ODEs was integrated with the procedure described in section 2.5.2.

All computations of this chapter were performed on desktop computers equipped with Intel(R) Xeon(R) CPU E31245 processors running at $3.30 \mathrm{GHz}$ and $16 \mathrm{~GB}$ to $32 \mathrm{~GB}$ of RAM. ${ }^{4}$ Because sequential runs do not require communication between cores they generally achieve the best performance per core. While this would be the most cost efficient choice, in this work the resulting waiting times for simulation results were

\footnotetext{
${ }^{3}$ In the original work [Ruc +15$] P_{l}$ was reported as $903 \mathrm{~nm} / \mathrm{s}$ which resulted from accidentally swapping $P_{p}$ and $P_{c}$ in eq. (5.10). Further the dimension of $P_{p}$ was wrongly given as $\mathrm{nm} / \mathrm{s}$.

${ }^{4}$ Those are quad core CPUs. Hyperthreading was enabled, but not used for computations.
} 


\begin{tabular}{|c|c|c|c|}
\hline Parameter & Symbol & Value & Unit \\
\hline Diffusion coefficient & $D_{c}$ & $223([$ AMS92]) & ${\mu \mathrm{m}^{2} / \mathrm{s}}^{2}$ \\
\hline Cytosolic resting $\mathrm{Ca}^{2+}$ & $c_{0}$ & 0.02 & $\mu \mathrm{M}$ \\
\hline ER resting $\mathrm{Ca}^{2+}$ & E & 700 & $\mu \mathrm{M}$ \\
\hline Diffusion coefficient & $D_{b}$ & 0.01 & ${\mu \mathrm{m}^{2} / \mathrm{s}}^{2}$ \\
\hline Total concentration & $B$ & 200 & $\mu \mathrm{M}$ \\
\hline On-rate & $k^{+}$ & 150 & $1 / \mu \mathrm{Ms}$ \\
\hline Off-rate & $k^{-}$ & 300 & $1 / \mathrm{s}$ \\
\hline $\mathrm{IP}_{3} \mathrm{R}$ pore radius & $R_{c}$ & 6 & $\mathrm{~nm}$ \\
\hline Channel flux coefficient & $P_{c}$ & $4.58 \times 10^{6}$ & $\mathrm{~nm} / \mathrm{s}$ \\
\hline Channel current & $I_{c}$ & 0.07 & $\mathrm{pA}$ \\
\hline SERCA pump coefficient & $P_{p}$ & 20,000 & $\mathrm{~nm} \mu \mathrm{M} / \mathrm{s}$ \\
\hline SERCA pump dissociation & $K_{d}$ & 0.05 & $\mu \mathrm{M}$ \\
\hline Leak flux coefficient & $P_{l}$ & 3.9 & $\mathrm{~nm} / \mathrm{s}$ \\
\hline Domain size & $\Omega$ & $3 \times 3 \times 2.5$ & $\mu \mathrm{m}^{3}$ \\
\hline \# of $\mathrm{IP}_{3} \mathrm{R}$ channels & $N$ & $4 \times 4$ & 1 \\
\hline inter channel distance & $d$ & 120 & $\mathrm{~nm}$ \\
\hline
\end{tabular}

Tab. 5.2: Model parameters of the reaction-diffusion equations. Note that the dimension of $P_{p}$ is different from the dimension of $P_{c}$ and $P_{l} .{ }^{3}$ 
not acceptable. Hence simulations were parallelized via MPI on up to 12 cores on 3 different nodes. For simulations with up to 4 cores inter process communication takes place via shared memory (RAM) of the single desktop computer and hence only involves minor overhead. However, parallelization to more cores (i.e. more than 1 node) requires communication via an ethernet connection and the overhead starts to exceed the gain. Distributing to more than three nodes (12 cores) did not speed up simulations any further. For all simulations the simulation time interval was set to $3000 \mathrm{~s}$. Since low $\left[\mathrm{IP}_{3}\right]$ involves lower channel activity those simulations were run on single nodes taking $500 \mathrm{~h}$ to $1800 \mathrm{~h}$ of CPU time. The simulation for $\mathrm{IP}_{3}$ of $80 \mathrm{nM}$ took $5000 \mathrm{~h}$ of CPU time distributed to 2 nodes ( 8 cores) yielding a total simulation duration of about one month.

One important consideration which has to be done before starting the simulations is which data should be saved to disk. Generally one would like to store as much information as possible which would allow to analyze a broad range of different aspects of the simulation in retrospective. In this section the full set of channel transitions was recorded, i.e. one can asses the channel states $X_{i j k}$ of all channels for every instant in time throughout the simulation interval. Storing the concentration fields of $\mathrm{Ca}^{2+}$ and the used buffer is more complicated. Since the simulations consisted of 150000 to 1500000 time steps and the spatial grid had around 37000 vertices, storing the full array of floating points would result in a memory consumption of $40 \mathrm{~GB}$ to $400 \mathrm{~GB}$ and turned out to be not reasonable. Instead the output was restricted to $\sim 4300$ nodes on the ER membrane. Further all time steps where $\Delta t$ to the previous time step was below $0.5 \mathrm{~ms}$ were filtered out. Those two filters reduced the amount of data by a factor 15 to 60. Figure 5.1 shows a snapshot of the dataset for the calcium concentration and the corresponding grid points on the membrane. Two overviews involving a combination of a calcium and a channel data set are depicted in fig. 5.2.

\subsection{Results}

The majority of the following subsections resemble the results already reported in [Ruc+15]. However, some additional unpublished aspects based on the same data are discussed as well.

\subsubsection{Domain size}

Because of the imposed no flux boundary conditions in eq. (5.5), $\mathrm{Ca}^{2+}$ can only be removed from the simulated cytosolic domain via SERCA pumps. Hence the domain size needs to be sufficiently large to avoid artificial $\mathrm{Ca}^{2+}$ accumulation during events 

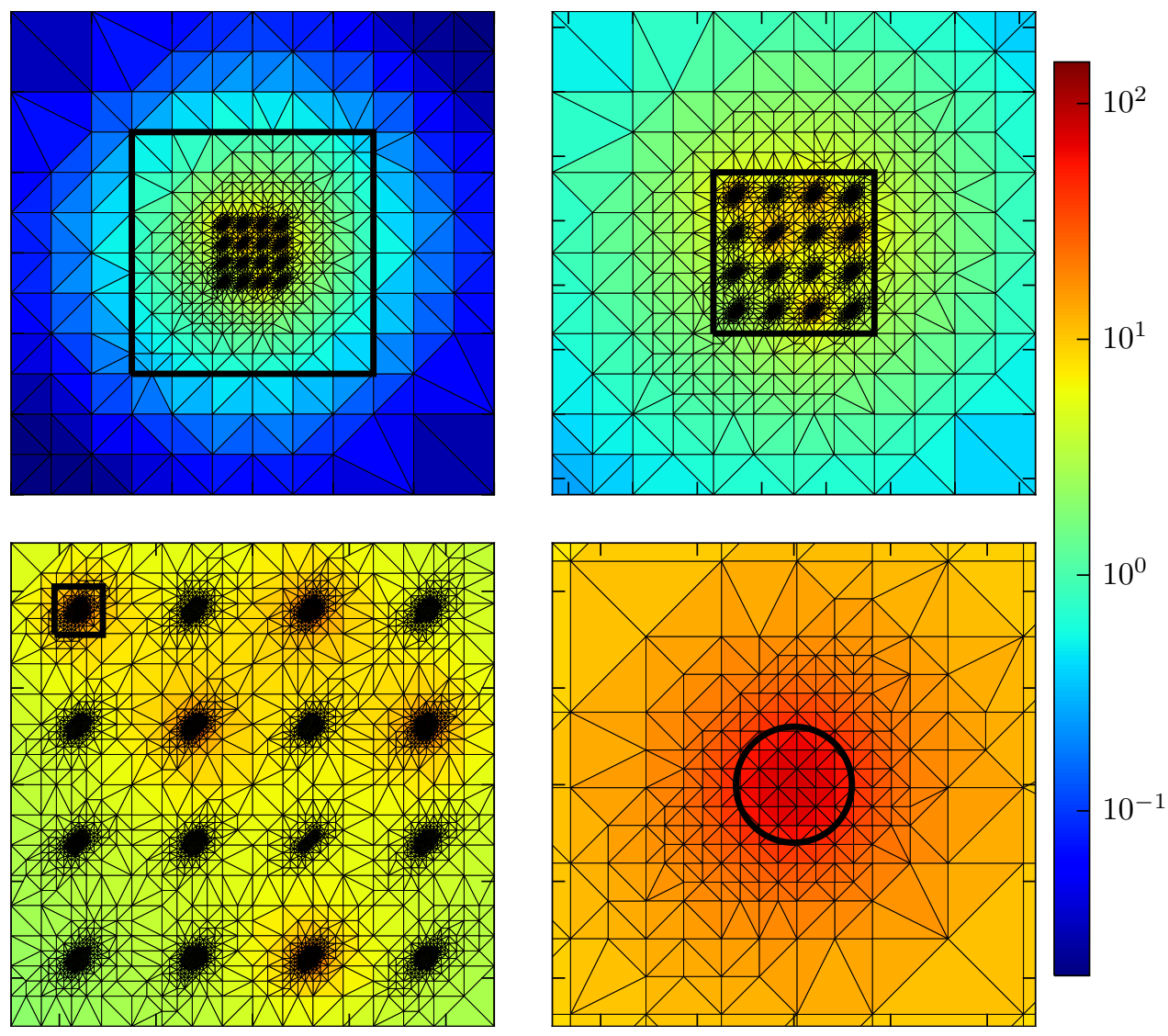

Fig. 5.1: Snapshot of the calcium profile of an open cluster. From top left to bottom right: full simulation domain $(3 \mu \mathrm{m} \times 3 \mu \mathrm{m})$, zoomed to $1.5 \mu \mathrm{m} \times 1.5 \mu \mathrm{m}$ around cluster, zoomed to $0.5 \mu \mathrm{m} \times 0.5 \mu \mathrm{m}$, and zoomed to $50 \mathrm{~nm} \times 50 \mathrm{~nm}$ around channel. The circle shows the channel pore radius $R_{c}=6 \mathrm{~nm}$. 


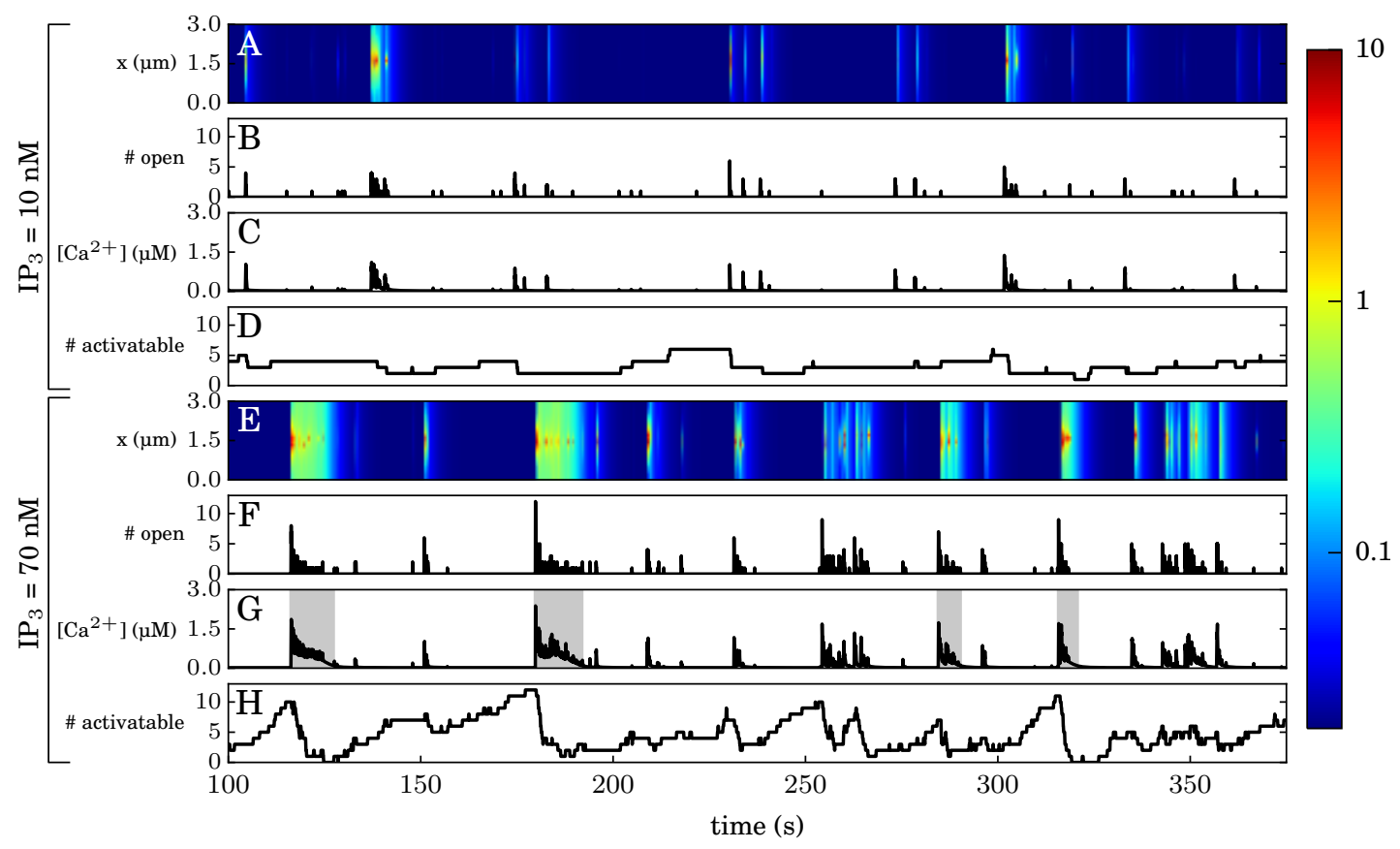

Fig. 5.2: Exemplary simulations for $\left[\mathrm{IP}_{3}\right]=10 \mathrm{nM}(\mathrm{A}-\mathrm{D})$ and $\left[\mathrm{IP}_{3}\right]=70 \mathrm{nM}(\mathrm{E}-\mathrm{H})$. In $\mathrm{A}$ and E the local $\left[\mathrm{Ca}^{2+}\right]$ "line scans" along a line on the ER membrane running through the cluster's center is shown. The color bar depicts the $\left[\mathrm{Ca}^{2+}\right]$ in $\mu \mathrm{M}$ and the warmer colors indicate the opening of channels. Because the line does not directly intersect with a channel pore, the nanodomain structure around a channel is not fully visible. The corresponding number of open channels is shown in $\mathrm{B}$ and $\mathrm{F}$. $\mathrm{C}$ and $\mathrm{G}$ display the average concentration in the cluster vicinity (500 nm box), D and $\mathrm{H}$ show the number of activatable channels, i.e., the number of channels that have at least three subunits with bound $\mathrm{IP}_{3}$. 


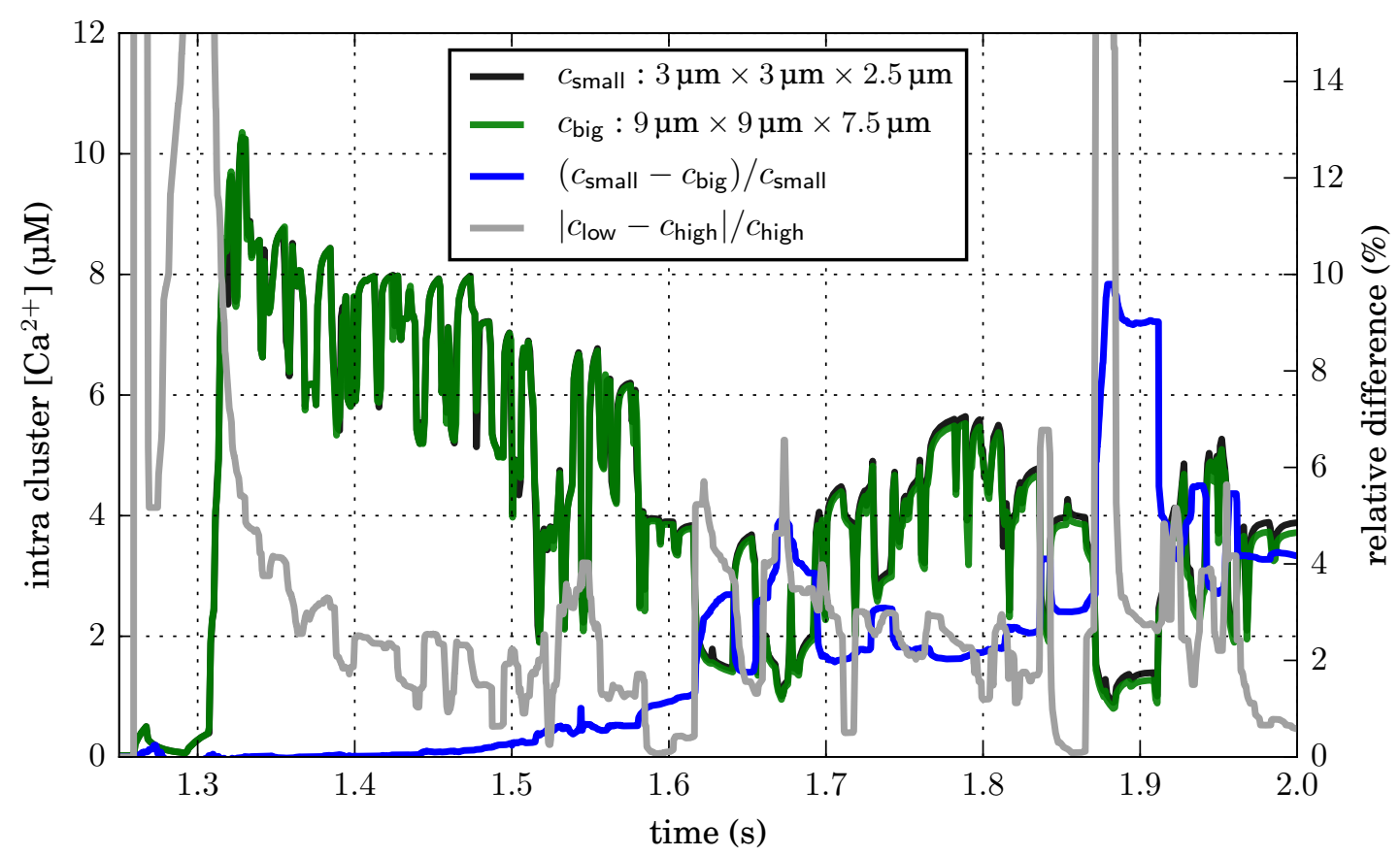

Fig. 5.3: Impact of domain size on calcium accumulation. The green and black traces denote the $\left[\mathrm{Ca}^{2+}\right]$ in the center or the cluster, directly at the ER membrane for a small and big bounding box, respectively. The right hand axis shows the relative difference between traces for the big and small bounding box and between traces of simulations with increased and decreased endogenous buffer concentration. The event corresponds to a replay of the first event of fig. $5.2 \mathrm{G}$.

with high $\mathrm{Ca}^{2+}$ efflux. Therefore, the first event from fig. 5.2 G was simulated in a much larger bounding box, in order to check the impact of the domain size on intra-cluster $\left[\mathrm{Ca}^{2+}\right]$. The course of channel openings and closings was predefined by the previously recorded course of the real stochastic events. As one can see from fig. 5.3, the absolute traces are almost identical. Further, the accumulation of calcium does not exceed a relative difference of $10 \%$.

The gray line in fig. 5.3 shows the relative difference between two other simulations where the endogenous buffer concentration was increased and decreased by $25 \%$ compared to the original simulation. This change of parameters induces around the same effect as changing the domain size. Hence, the accumulation effect due to a finite domain size is considered tolerable with respect to the uncertainties which are induced by other parameters of the model. 

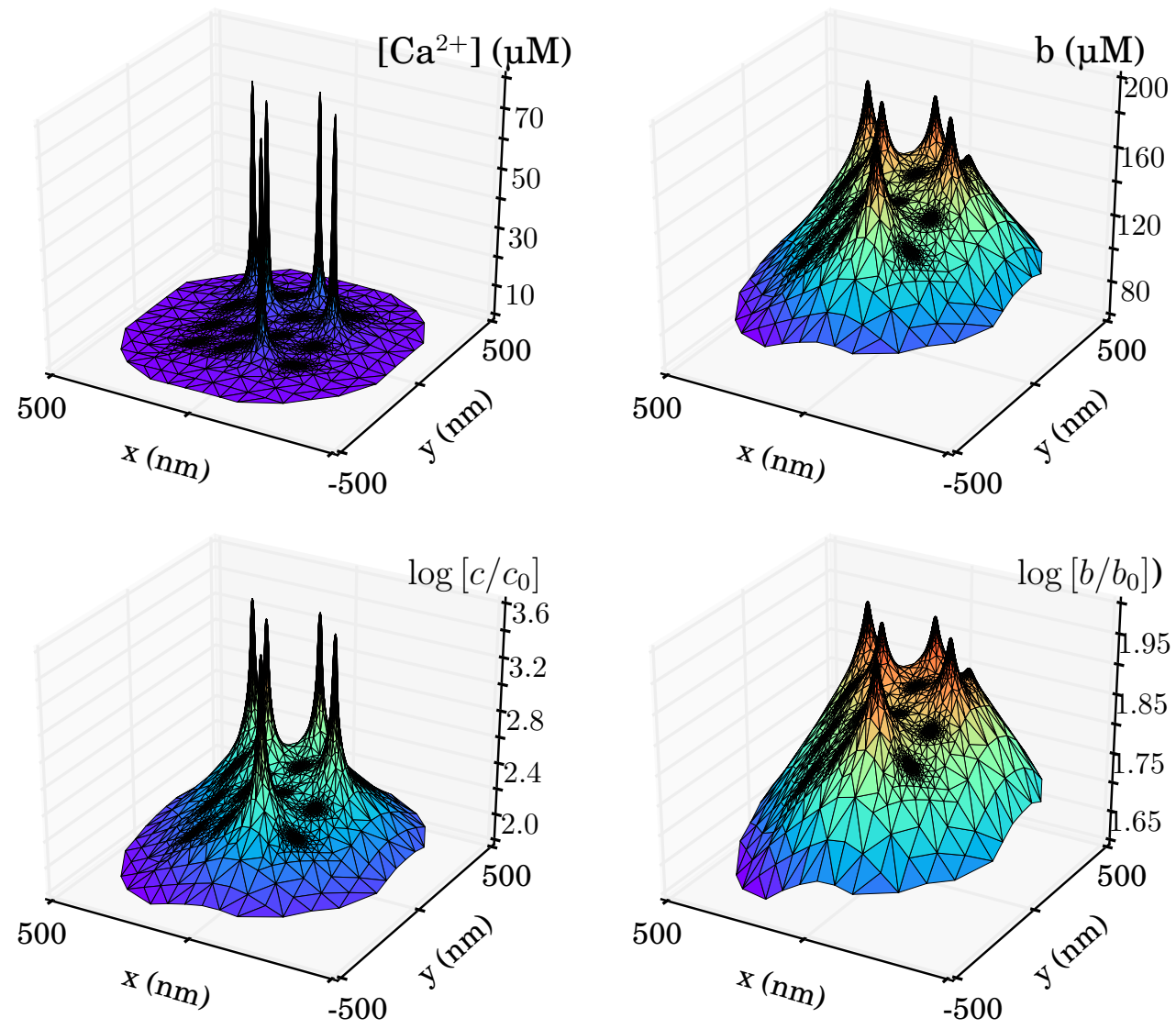

Fig. 5.4: Concentration profiles of $\mathrm{Ca}^{2+}$ (left) and bound buffer (right) for linear (upper) and logarithmic (lower) scale. For the logarithmic scale, concentrations where normalized to rest levels $c_{0}$ and $b_{0} \approx 2 \mu \mathrm{M}$, see eq. (5.3).

\subsubsection{Micro- and Nanodomain concentration profiles}

The color coding from fig. 5.1 already indicates the huge gradients of the $\mathrm{Ca}^{2+}$ profiles around channel pores and the much larger $\mathrm{Ca}^{2+}$ structure around the whole cluster. Figure 5.4 shows the concentrations of $\mathrm{Ca}^{2+}$ and the bound stationary buffer with both, linear and logarithmic scale. Here, the importance of a locally refined grid around the channel pores becomes even more apparent. Further, one can clearly distinguish the nano scale domains around individual channels and the micro scale concentration profile around the cluster. As presented in fig. 20 of $[\operatorname{Rud}+10]$, the level of expression of the microdomain depends on the used buffer settings. 


\subsubsection{Event definition and lifetime}

In section 4.3 some general aspects of collective events (CEs) were discussed. In the following section, the definition of a $\mathrm{CE}$ will be as follows. First, the temporal evolution of the spatially averaged $\left[\mathrm{Ca}^{2+}\right]$ in the cluster vicinity $c_{\text {avg }}$, a $500 \mathrm{~nm}$ cube around the center of the cluster (see fig. 5.1, upper right and lower left), is calculated. A CE starts whenever a channel opens, given there is no other $\mathrm{CE}$ of the cluster at hand. It lasts as long as $c_{\text {avg }}$ exceeds a threshold value of $0.1 \mu \mathrm{M}$, or if this threshold never gets surpassed, until the last channel closes. From the resulting set of start and end times, all CEs containing only a single channel opening, and als CEs which last less than $15 \mathrm{~ms}$ were filtered out. Using the spatial average of $\left[\mathrm{Ca}^{2+}\right]$ to define a CE serves to facilitate comparison to experiments on $\mathrm{Ca}^{2+}$ puffs and waves in cells with dye buffer, where the number of open channels underlying a release event is not directly known. Events according to this definition are depicted as gray background in fig. 5.9, red vertical lines denote event starts. Note how the high residual $\mathrm{Ca}^{2+}$ ensures that correlated fluctuations get merged into one large $\mathrm{CE}$ (lower), while in the upper case 4 individual CEs are detected.

The above definition allows to measure the distributions of event lifetimes for different $\mathrm{IP}_{3}$ concentrations. The distributions for $10 \mathrm{nM}$ (red bars), $35 \mathrm{nM}$ (green bars) and $70 \mathrm{nM}$ (blue bars) are shown in fig. 5.5. For $10 \mathrm{nM}(20 \mathrm{nM})$ only 3 of 90 events (10 of 166) lasted longer than $2 \mathrm{~s}(3 \mathrm{~s})$ corresponding to $3.3 \%(6.0 \%)$, whereas for $\left[\mathrm{IP}_{3}\right]=80 \mathrm{nM}$, 72 of 309 events lasted longer than $3 \mathrm{~s}$ $(23 \%)$. Summarizing, for low $\left[\mathrm{IP}_{3}\right]$ the lifetime does not exceed one or two seconds. The average duration of $0.5 \mathrm{~s}$ for $10 \mu \mathrm{M}$ is similar to puff durations measured in experiments from Xenopus oocytes [CP00].

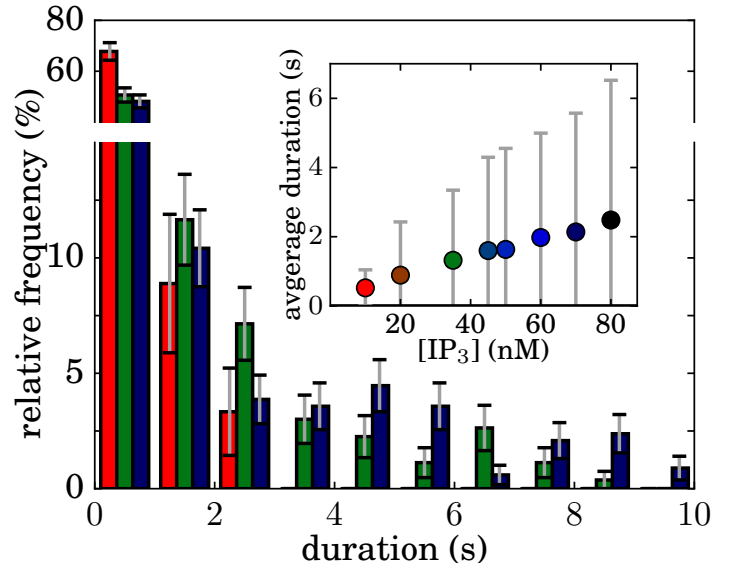

Fig. 5.5: Distribution of event durations for different $\mathrm{IP}_{3}$ concentrations. Error bars denote the sampling error of the histogram. The inset shows the average event duration and its standard deviation.

For high concentrations of $\mathrm{IP}_{3}$, one additionally observes long lasting release events of up to $10 \mathrm{~s}$. While the majority of events (more than $60 \%$ ) was shorter than $1 \mathrm{~s}$ for all concentrations, the distribution shows a growing shoulder from $35 \mathrm{nM}$ onwards. This change of type of distribution also gets reflected by an increased average and variance of the event lengths as shown in the inset of fig. 5.5. 


\subsubsection{Event lifetime correlates with IP3 binding}

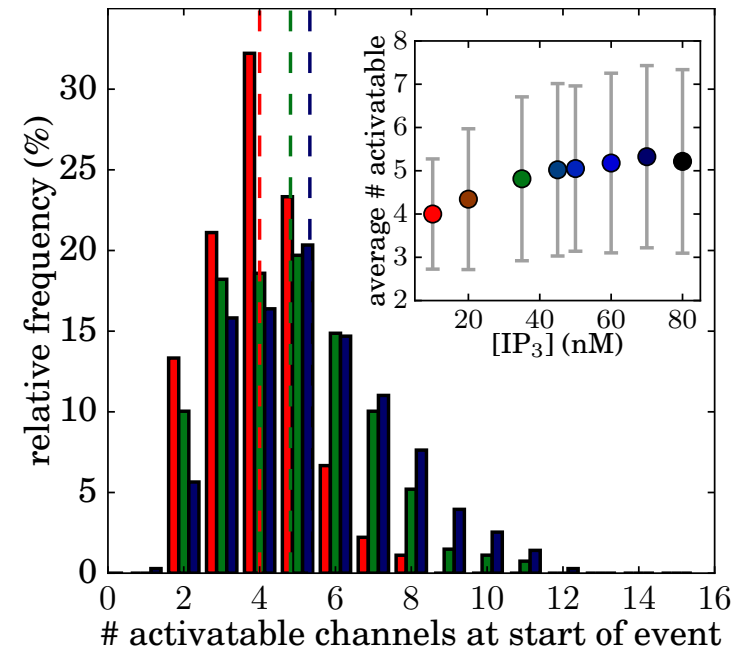

Fig. 5.6: Distribution of the number of activatable channels at the beginning of the events for different $\left[\mathrm{IP}_{3}\right]$. Dashed lines mark the mean value. Inset: The average number of activatable channels at the beginning of an event. Vertical bars are standard deviations.

A straight forward approach to check the interplay of lifetime and number of activatable channels is to measure the number of activatable channels at the beginning and at the end of an event and itemize these numbers with the event lifetime and vice versa. In fig. 5.6 the distribution of the number of activatable events at start is depicted for $10 \mathrm{nM}, 35 \mathrm{nM}$, and $70 \mathrm{nM}$ in red, green, and blue, respectively. One can immediately grasp the increased chance to have many activatable channels for higher $\mathrm{IP}_{3}$ concentrations, whereas the chances of having more than 6 activatable channels for $10 \mathrm{nM}$ are close to zero. Interestingly, the average number of activatable channels does not change in such a large extent as demonstrated by the inset. In fact, there appears to be some saturation in the average number starting from around $50 \mathrm{nM}$. The inset also reveals the increasing variance, i.e. the broadening of the distribution for increasing $\left[\mathrm{IP}_{3}\right]$.

The effect of a higher number of activatable channels becomes clear from fig. 5.7. More or less independently of the $\left[\mathrm{IP}_{3}\right]$, event lifetime increases significantly when more channels have a sufficient amount of bound $\mathrm{IP}_{3}$ upon event start $(\mathrm{A})$. The curve looks different only for $10 \mathrm{nM}$, since in that case no long events have occurred at all. Further, one can see from fig. $5.7 \mathrm{~B}$ that long events very likely render the cluster completely non activatable. 

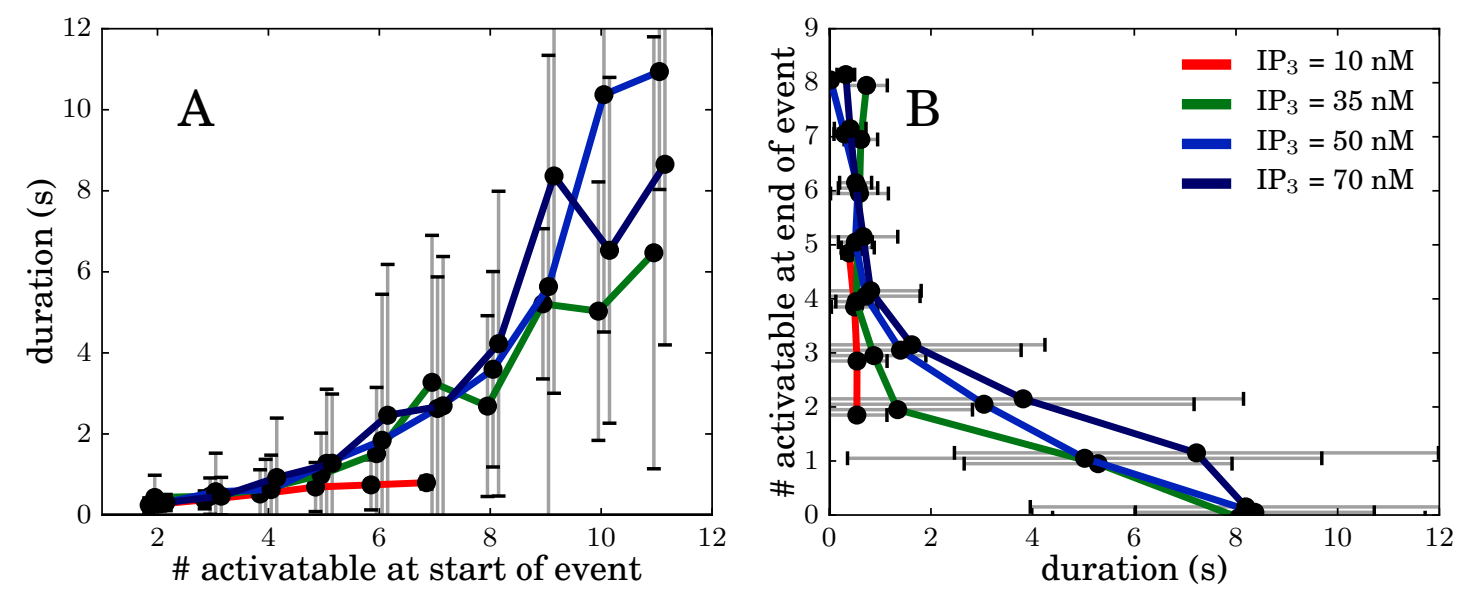

Fig. 5.7: (A) Event duration depends on the number of activatable channels at the beginning of an event. While low numbers of activatable channels very likely will lead to short events, many activatable channels not necessarily lead to long events. (B) The number of activatable channels at the end of an event as a function of the duration. Bars denote standard deviations. Absent bars indicate single observations.

Summarizing, many activatable channels at event start will likely result in high event lifetime, which likely results in a completely non activatable cluster. What remains is the question how, and why $\mathrm{IP}_{3}$ promotes such a cycle, and why this effect disappears for low $\mathrm{IP}_{3}$ levels. I.e. where does the increase of variability in fig. 5.6, which ultimately promotes higher excitability at event start, originate from? In a probabilistic description the explanation is straight forward: The number of activatable channels essentially defines the clusters excitability, i.e. the probability that, given all channels are closed, any channel will open within the next instant of time. Starting from a completely non excitable cluster, the excitability would rise to its saturation level where all channels are activatable. However, this process will be repeatedly interrupted when channels open, and the interruption rate itself is again proportional to the clusters excitability. 
Figure 5.8 shows examples for such a processes with two different excitation rates $\alpha$ (high $\alpha$ corresponds to high $\mathrm{IP}_{3}$ ) and is based on the following assumptions.
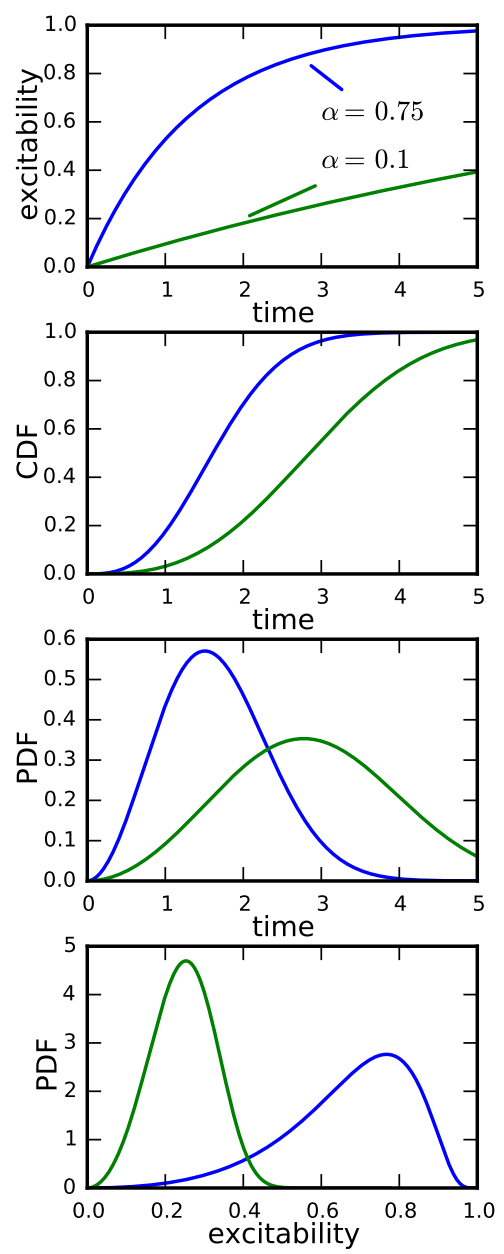

Fig. 5.8: From top to bottom: eqs. (5.11), (5.13), (5.15) and (5.17) for two different excitation rates $\alpha$.

First, the excitability rate grows like

$$
r(t)=1-\exp [-\alpha t]
$$

where $r(t)=1$ is equivalent to a fully activatable cluster. Since $r(t)$ is monotone, one can calculate the inverse function:

$$
t(r)=-\frac{\log [1-r]}{\alpha}
$$

Further, the waiting time distribution (CDF) follows from eqs. (2.12) and (2.13) with integration:

$$
P_{\text {wait }}(t)=1-\exp \left[-\frac{1}{2} t^{2}+\frac{1-\exp [-\alpha t](\alpha t+1)}{\alpha^{2}}\right] \text {. }
$$

The PDF for observing a given time $t$ reads as

$$
\begin{aligned}
\rho_{\text {time }}(t) & =\frac{\mathrm{d} P_{\text {wait }}}{\mathrm{d} t}= \\
& =t \exp \left[\frac{1-e^{-\alpha t}}{\alpha^{2}}-\frac{t e^{-\alpha t}}{\alpha}-\frac{1}{2} t^{2}\right]\left(1-e^{-\alpha t}\right),
\end{aligned}
$$

and with a change of the dependent variable according to

$$
f_{Y}(y)=\left|\frac{d}{d y}\left(g^{-1}(y)\right)\right| \cdot f_{X}\left(g^{-1}(y)\right)
$$

the PDF for the occurrence of different excitabilities $r$ follows as:

$$
\rho_{\text {exc }}(r)=\frac{1}{\alpha(1-r)} \rho_{\text {time }}(t(r)) .
$$

One can see, that for higher excitation speed the probability distribution shifts to higher excitability rates, just as in fig. 5.6. 

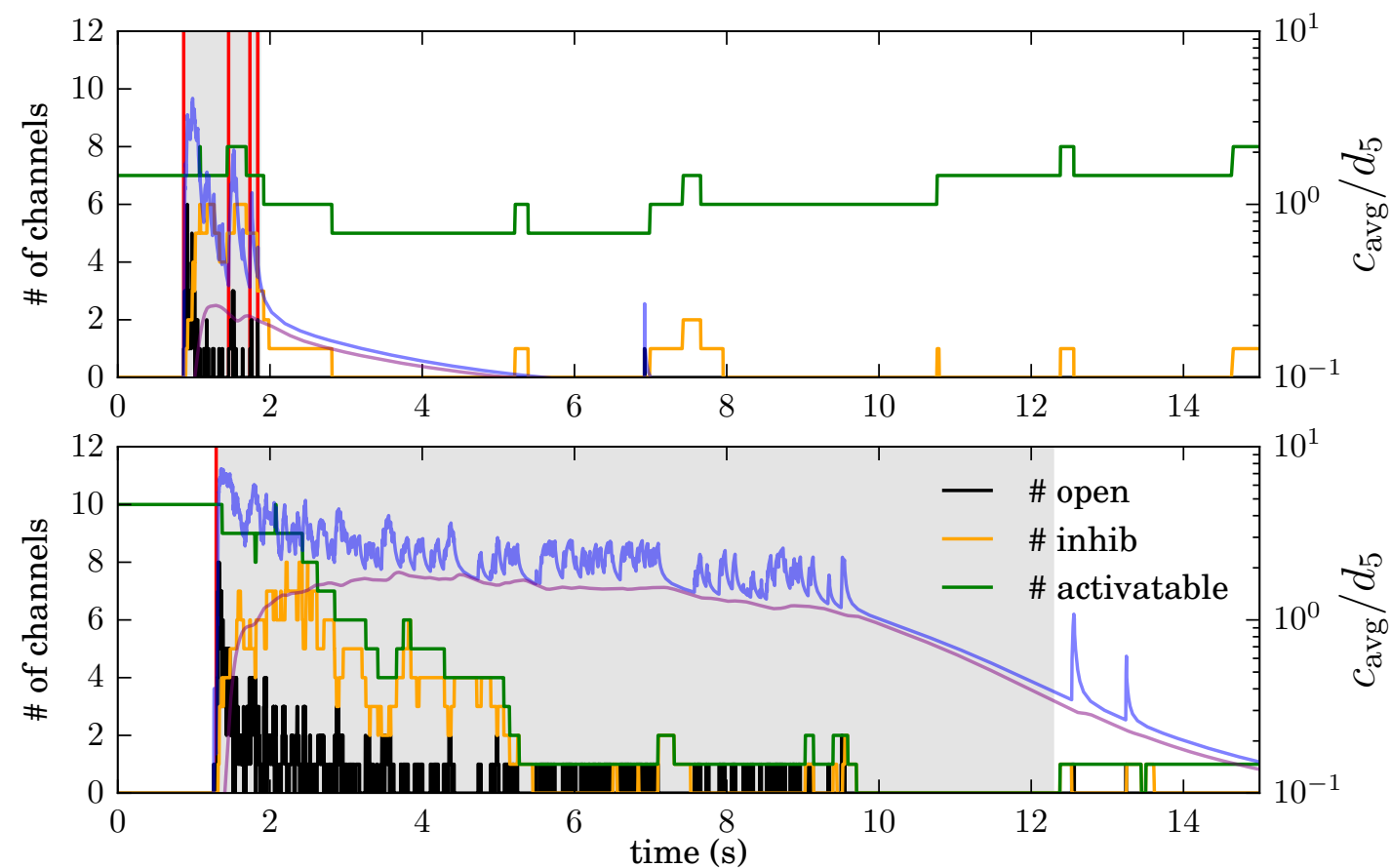

Fig. 5.9: Inhibition can only terminate events which do not accumulate large amounts of residual $\mathrm{Ca}^{2+}$. The blue line depicts the average concentration of fee $\left[\mathrm{Ca}^{2+}\right]$ in a $500 \mathrm{~nm}$ box around the cluster center $\left(c_{\text {avg }}\right)$ measured as multiples of the DYK activation coefficient $d_{5}=0.25 \mu \mathrm{M}$. The purple line depicts the $\left[\mathrm{Ca}^{2+}\right]$ at the edge of the domain. Since the timescale of de-inhibition is $b_{2}=1.56 \mathrm{~s}^{-1}$ channels will frequently reopen when the residual $\left[\mathrm{Ca}^{2+}\right]$ is to high. The two events correspond to the events at $150 \mathrm{~s}$ and $120 \mathrm{~s}$ from fig. $5.2 \mathrm{~F}$, respectively. 


\subsubsection{Inhibiton failure and loss of IP3}

The reason for the increase in event lifetime is presented in fig. 5.9. It shows the number of open, inhibited and activatable channels on the left axis, as well as the average concentration in cluster vicinity $c_{\text {avg }}$ (blue, right axis). Recall from table 3.1 that the activation coefficient of the DYK model is $d_{5}=0.25 \mu \mathrm{M}$. For events with less activatable channels at event start, the average concentration does not rise that high, and also decreases faster, while for many open channels at start, a large residual calcium remains after the initial peak of channel openings. This residual calcium hinders the termination of the event by inhibition, since deinhibited channels will frequently reopen. Further, the dissociation coefficient for $\mathrm{IP}_{3}$ while inhibited, $d_{3}=2 \mu \mathrm{M}$, is much higher than while not inhibited $\left(d_{1}=0.001 \mu \mathrm{M}\right)$, i.e. channels equilibrate towards unbound $\mathrm{IP}_{3}$. When they have lost $\mathrm{IP}_{3}$ they are unlikely to unbind inhibitory $\mathrm{Ca}^{2+}\left(X_{001} \rightarrow X_{000}\right.$ or $X_{011} \rightarrow X_{010}$ ) because these transitions are hindered by residual $\mathrm{Ca}^{2+}$.

\subsubsection{Classification of collective events in puffs and waves}

The diversity in shape and duration of the simulated release events as a function of $\left[\mathrm{IP}_{3}\right]$ encourages the attempt to relate them to the different release patterns found in experiments: puffs and waves (see also section 3.2). Usually, short-termed puffs are observable for all concentrations of $\mathrm{IP}_{3}$ [BLB00; Cao+13; Swi+99]. Long lasting and potentially propagating (or global) release events are termed waves and only occur for higher $\left[\mathrm{IP}_{3}\right][\mathrm{CP} 00 ; \mathrm{MP} 01 ; \mathrm{Dup}+11]$. A plausible approach is to follow the same binary classification into puffs and waves, as the observed results show similar features. Optimally, the classification criterion would clearly distinguish the short release events, which get terminated by inhibition, from the events which show the long activity tails and terminate due to $\mathrm{IP}_{3} \mathrm{R}$ unbinding. Notably, the simulated setup only consists of a single cluster and therefore cannot exhibit any real spatial waves or global oscillations. However, the shape of the second class of CEs suggest that their extended release might actually be able to couple to other clusters of $\mathrm{IP}_{3} \mathrm{R}$ via CICR. This also means, that the classification criterion should reflect the "global aspect" of the experimental waves: "waves" should have the potential to trigger release from another close-by imaginary cluster. Therefore a $\mathrm{CE}$ is classified as wave if it induces a local $\left[\mathrm{Ca}^{2+}\right]$ of at least $0.25 \mu \mathrm{M}$ (corresponding to the dissociation constant of activation, $d_{5}$, see table 3.1 ) at the edge of the domain. For simplicity this concentration gets measured at one corner of the simulation box which is approximately $2 \mu \mathrm{m}$ afar from the cluster center. Otherwise the event is classified as puff.

Exemplary results of the above criterion can be seen in fig. 5.9, where in the upper 
case 4 individual puffs are detected, while the lower event shows one single wave. The concentration at the edge of the domain is depicted in purple. Further, in fig. 5.2 G, waves are indicated by a shaded background. Interestingly, the $\mathrm{Ca}^{2+}$ signals between $250 \mathrm{~s}$ and $275 \mathrm{~s}$ in fig. $5.2 \mathrm{G}$ clearly differ from the homogeneous signals produced by waves. With the above criterion, the burst of channel openings is not classified as wave, but as a sequence of distinct puffs.

Figure 5.10 shows an "average" puff and an "average" wave demonstrating how well the separation into puffs and waves actually works. The average was calculated over the number of open channels for puffs and waves individually. Then, the curves were normalized to a peak value of one. The solid red line shows the resulting average puff for $\left[\mathrm{IP}_{3}\right]=10 \mathrm{nM}$ and resembles the fact, that for this concentration only puffs were observed. Its simple exponential decay represents puff termination by inhibitory $\mathrm{Ca}^{2+}$ on a fast time scale. In contrast to that, for $70 \mathrm{nM}$, the dotted blue line shows a second release phase for times

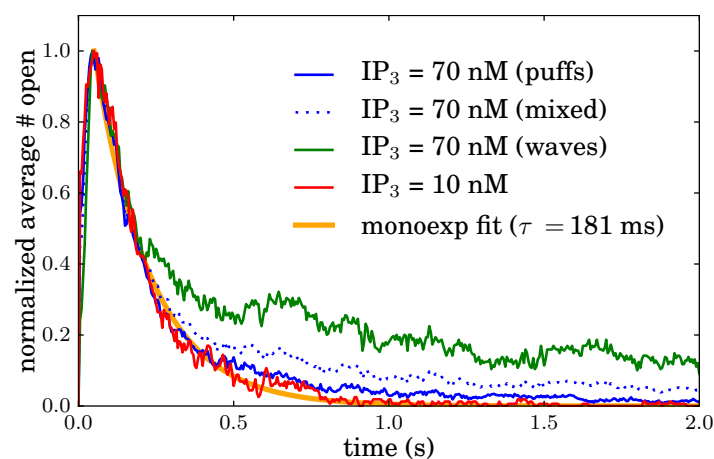

Fig. 5.10: Average event shapes of waves and puffs. To compare difference $\left[\mathrm{IP}_{3}\right]$, peaks were normalized to 1 . For high $\left[\mathrm{IP}_{3}\right]$, event classification into waves and puffs nicely separates the termination mechanisms into fast inhibition for puffs, and a mix of inhibition and slow $\mathrm{IP}_{3}$ inactivation for waves.

over $200 \mathrm{~ms}$ which clearly deviates from the profile of puffs. If one splits the population of CEs for $70 \mathrm{nM}$ into the two subgroups, the group of puffs nicely matches the curve for $10 \mathrm{nM}$, while the group of waves shows the characteristic low activity trail. Analyzing the event shape for waves, one can clearly distinguish the two termination mechanisms. The first $200 \mathrm{~ms}$ are dominated by inhibition, which gets followed by the slow $\mathrm{IP}_{3}$ unbinding, providing a backup process if residual $\mathrm{Ca}^{2+}$ forbids termination due to inhibition.

With the above definition of puffs and waves, one can also measure the distribution of inter wave intervals (IWIs) and inter puff intervals (IPIs). An IPI, or IWI, is simply defined as time between the end of the preceding and the beginning of the proceeding puff or wave, respectively. If two puffs were split by a wave, the corresponding IPI was discarded. The resulting average values, and their standard deviations are depicted in fig. 5.11 as a function of $\left[\mathrm{IP}_{3}\right]$. Similar as observed in experiments [MP01], the model predicts decreasing IWI for increasing $\left[\mathrm{IP}_{3}\right]$. Further, the IWIs are similar to periods of global oscillation measured in various cell types [MP01; Thu+12; Dup+11]. In fact, 


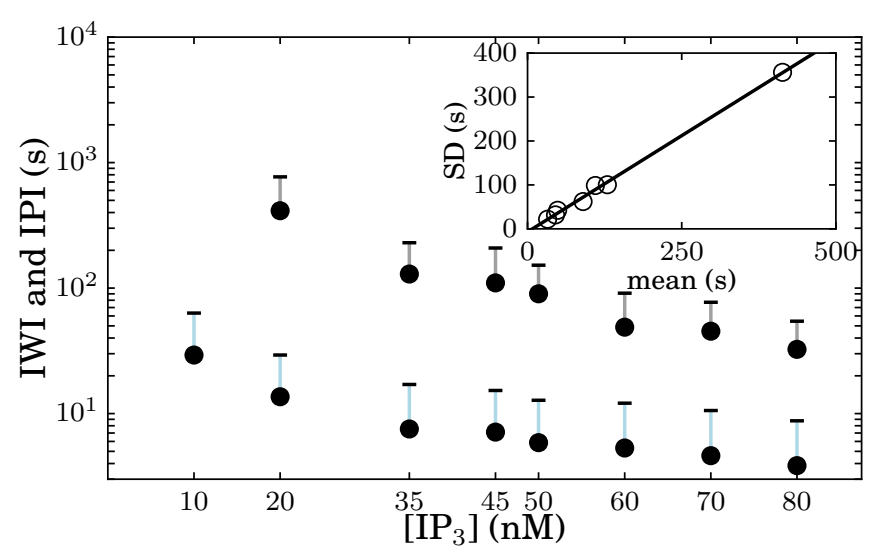

Fig. 5.11: Average IWI (black) and IPI (blue) for different $\left[\mathrm{IP}_{3}\right]$. For $\left[\mathrm{IP}_{3}\right]=10 \mathrm{nM}$, no waves could be observed. The inset shows a linear correlation between the average and SD of the IWI similar as reported by $[\mathrm{Sku}+08]$.

recent experiments by Thurley et al. [Thu+14] even show a exponential scaling of the period with stimulation, which is consistent with the scaling of IWIs in fig. 5.11.

The inset of fig. 5.11 whos the linear correlation between the average and SD of the IWI, which was also observed in experiments [Thu+12]. The resulting coefficient of variation $(\mathrm{CV})$ is decreasing from $\sim 0.9$ for $\left[\mathrm{IP}_{3}\right]=10 \mathrm{nM}$ to $\sim 0.5$ for $80 \mathrm{nM}$. The slope of the regression line is 0.88 and the minimal average IWI (i.e., the intersection with the $x$-axis) is $17 \mathrm{~s}$ which is similar to what was found for several cell types including astrocytes $[\mathrm{Sku}+08]$. In the case of coupling of multiple clusters, synchronization may lead to higher regularity of release events [PRK01]. This may explain why smaller variabilities were reported for human embryonic kidney (HEK) cells [Sku+08; Thu+14], Hepatocytes [DAC08] and Xenopus oocytes [Cal+98; Sun+98]. 


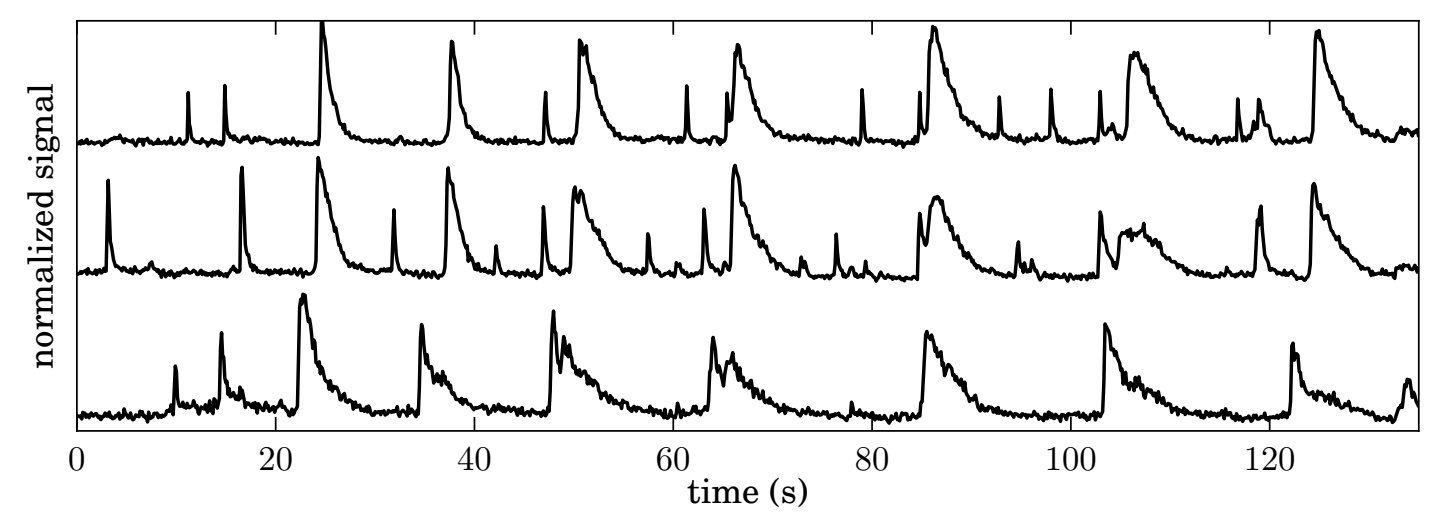

Fig. 5.12: Representative fluorescence traces from 3 puff sites in Xenopus oocytes. Waves were identified as large signals that occurred approximately at the same time at all cluster sites.

\subsubsection{Inter wave dynamics and comparison with experimental data}

The experimental data presented in this section was generously provided by Jonathan S. Marchant and Ian Parker and was first published in $[\mathrm{Ruc}+15]$. The experimental procedures are described in [MP01], exemplary traces are provided in fig. 5.12.

From fig. 5.12 one can see what was also reported before in [MCP99; MP01]: in Xenopus oocytes puffs appear in the phase between succeeding global waves. The waves are identified as a group of large events which appear at all cluster sites at approximately the same time. The small events which can not be itemized to a wave are then identified as puffs. In the following, experimental event amplitudes correspond to the peak amplitudes of puffs and waves, while for simulated data the peak number of open channels is denoted as peak amplitude.

In section 5.3.4 the $\mathrm{IP}_{3}$ rebinding process between waves was already discussed. Now, the phase of puffs between waves, both in experiments and simulations will be compared.
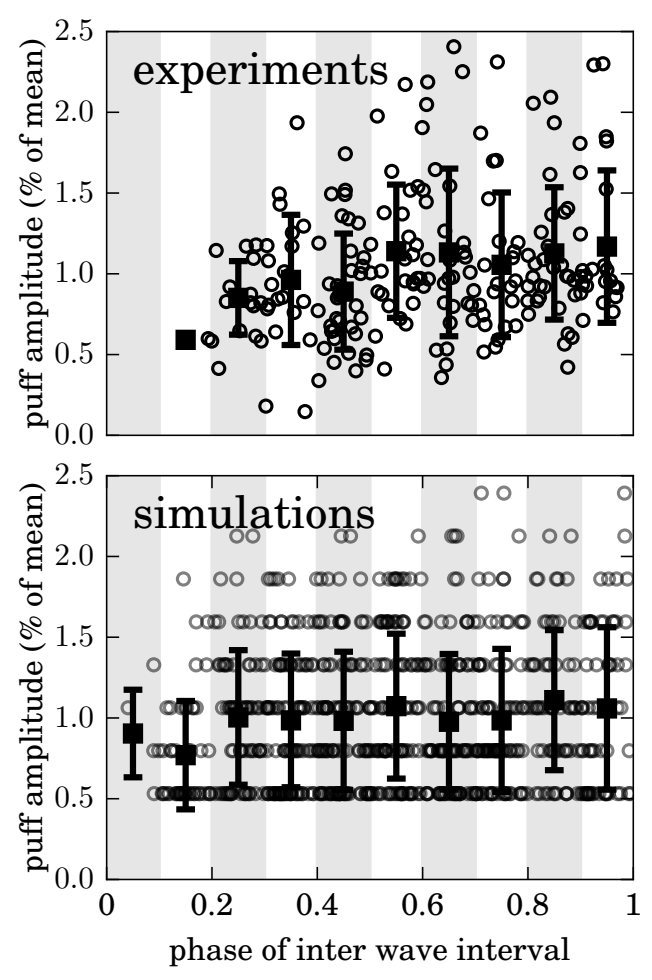

Fig. 5.13: Scatter plot of puff amplitudes. The data was grouped (shaded regions), black markers and error bars denote the mean and standard deviation of each group, respectively. 
Therefore the phase of a puff is defined as the ratio of time which has passed since the last wave has terminated to the total interval between the wave events which surround a puff. As seen from fig. 5.11, the three data sets for high $\left[\mathrm{IP}_{3}\right]$ are quite similar, hence simulation data was pooled for $60 \mathrm{nM}, 70 \mathrm{nM}$, and $80 \mathrm{nM}\left[\mathrm{IP}_{3}\right]$. Experimental data was pooled from four experiments. The amplitudes of experimental and simulated data were then rescaled to their respective mean amplitudes. Figure 5.13 shows the relative amplitudes of individual puffs and their corresponding phases for experiments and simulations. Further, the IWIs were binned into 10 equal bins and mean values and standard deviations were calculated per bin.
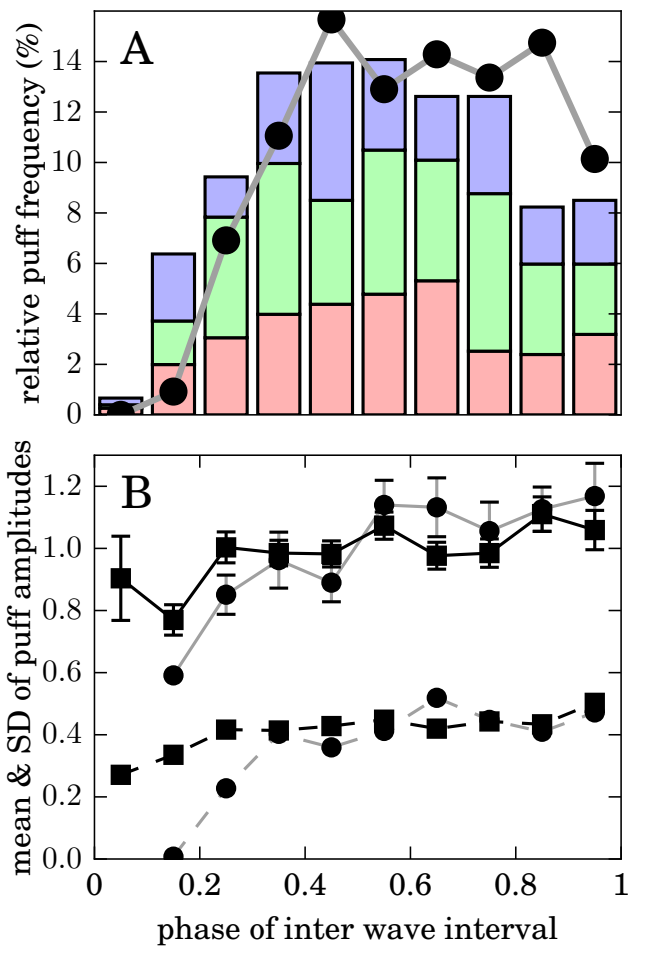

Fig. 5.14: (A) Temporal distribution of puff frequency between two waves after binning of data in 0.1 phases. (B) Mean (solid lines with standard error) and standard deviations (dashed lines) of binned amplitudes for simulations (black) and experiments (gray).

From Figure 5.13 one can already suspect some refractory time of the clusters after wave termination. To make this even more clear, fig. 5.14 A shows the puff frequency, i.e. the percentage of puffs occurring in a specific bin w.r.t. the total number of puffs. The colored bars represent the simulated data itemized according to $\left[\mathrm{IP}_{3}\right]$ in red, green, and blue for $60 \mathrm{nM}, 70 \mathrm{nM}$, and $80 \mathrm{nM}$, respectively. The gray line resembles experimental data. Figure $5.14 \mathrm{~B}$ compares the course of mean and SD from fig. 5.13 for experiments (gray) and simulations (black).

All together one can make the following observations from figs. 5.13 and 5.14. First, there clearly is a silent phase proceeding wave termination during which no puffs occur. It is apparent in both, experimental and simulated data and lasts around 20\% (10\% for simulations) of the IWI. Second, there is a noticeable increase in SD of puff amplitudes from close to 0.2 to 0.5 . More importantly, there is a small number of large amplitude events that are absent in the first half of the IWI but are present in the second half. These events likely represent the large elementary releases that possess the potential to evolve into a wave, but still fail to do so due to the inherent noise of the gating process. Third, the refractory effect is much smaller for the amplitude of puffs 
than for their frequency. And finally, there appears to be a decrease of puff frequency towards the end of the events in both simulations and experiments. This decrease may also be related to the second observation and can be explained as follows: A future wave may require some preceding "silent phase" where the accumulation of activatable channels is not interrupted and partially reset by puffs. Experiments in [MP01, Fig. 3] indicate that this effect is more clear for cells with moderate and large IWI.

\subsection{Discussion}

From the experimental perspective the coexistence of puffs and waves for large $\left[\mathrm{IP}_{3}\right]$ is well documented since quite some time [MCP99; MP01]. Despite the fact that the simulated system of this chapter only consisted of a single cluster, two distinct release event types were found for that cluster. One of the release patterns could clearly be identified as puffs. The other release type was henceforth termed "wave". Obviously a single cluster can not exhibit any real calcium wave, such as reported from multi cluster simulations or experiments. Still, the term wave was chosen to clearly separate this release pattern from puffs and because of its possible capability to couple to imaginary distinct clusters. The intriguing feature of the results presented in this chapter is the fact that diverging event types, i.e. puffs and waves, may be present already on a single cluster level. While other models showing this coexistence usually require multiple clusters where waves emerge due to synchronization and coupling effects [Fal03], this chapter demonstrated how the binding state of $\mathrm{IP}_{3}$ may serve as a secondary excitability state and thereby provides an alternative explanation to the emergence of waves, already on the single cluster scale.

The hypothesis of $\mathrm{IP}_{3}$ unbinding during long lasting release events, which originally was proposed in [RJS12], was investigated in the first parts of this chapter. It turned out that $\mathrm{IP}_{3} \mathrm{R}$ can lose $\mathrm{IP}_{3}$, and that this loss depends on the course of the release events. The loss of $\mathrm{IP}_{3}$ can thereby serve as a secondary inhibitory feedback to CICR which terminates release if inhibition by $\mathrm{Ca}^{2+}$ fails. Finally, in section 5.3 .7 it is demonstrated that not only simulations, but also experiments support the hypothesis of $\mathrm{IP}_{3}$ unbinding during waves. Both datasets show some key features which can be expected from the hypothesis.

Similar experiments reported correlations of the inter puff time with the preceding puff amplitude [DP13]. There the correlations were accounted to inhibitory $\mathrm{Ca}^{2+}$ binding to the $\mathrm{IP}_{3} \mathrm{R}$, however, the results presented here also allow for a different interpretation: $\mathrm{IP}_{3}$ unbinding can induce similar correlations and also provides an answer for the unexpected variability of puff amplitudes [DP13]. 
One critical thought to keep in mind is the fact that simulated data of a single cluster was compared to experiments covering coupled clusters. Synchronization effects emerging from larger systems containing multiple clusters may increase the regularity of oscillations and therefore exaggerate the synchronization effects of dynamic $\mathrm{IP}_{3}$ binding and unbinding, as is often the case when coupling oscillating systems [PRK01]. Hence, if the hypothesis of $\mathrm{IP}_{3}$ during sustained release is correct the resulting effects might be more pronounced in the experimental data. This is a possible explanation for some of the exaggerated effects presented in section 5.3.7.

With the above in mind, the simulations are a proof of concept that dynamic $\mathrm{IP}_{3}$ binding actually can provide an alternative explanation for the emergence and termination of waves and may have a significant impact on the regulation of $\mathrm{IP}_{3}$ on timescales of seconds. While using only a single cluster in the simulations (dictated by limitations of computational power), the insights gained about the dynamics of an isolated system can serve as a building block and thereby help elucidate the global dynamics of coupled systems. Therefore, the next chapter will present results of a model with dynamic $\mathrm{IP}_{3}$ binding, but coarse grained $\mathrm{Ca}^{2+}$ dynamics, allowing to couple many clusters with reasonable computational effort. 


\section{Chapter 6}

\section{Coarse grained multi cluster model}

In section 5.1, the complexity of the coupled partial differential equations poses a big obstacle for the investigation of statistical properties of clusters of $\mathrm{IP}_{3} \mathrm{R}$ channels ${ }^{1}$. Because the number of $\mathrm{IP}_{3}$ channels and the required simulation interval both multiply the required computation power, the previous model is not suited to gather sufficient statistics. This section will introduce a coarse grained model (CGM), which avoids the effort that is accompanied with the HDM by reducing the description of $\left[\mathrm{Ca}^{2+}\right]$ to a set of coupled ODEs. The CGM was not designed following any specific biological motivations, instead the three major goals were:

1. Phenomenological. The previous chapter has demonstrated that the evolution of $\left[\mathrm{Ca}^{2+}\right]$ is of key importance for the statistical properties of the system. The new model should capture the main aspects of these dynamics qualitatively, not quantitatively.

2. Numerical simplicity. Computations should be very fast for a single cluster (a couple of hours at maximum). This allows to run larger systems, gather more statistics and check many different parameter combinations.

3. Reduced set of parameters. Many parameters of the old model have similar effects on the dynamics of the system. The new set of parameters should combine the old ones in an "orthogonal basis".

\footnotetext{
${ }^{1}$ Gathering statistics for a specific set of parameters often takes days or weeks, i.e. one cannot quickly check for some hypothesis.
} 
To achieve the first goal, it makes sense to study the results of the previous numerical simulations, both in this work from other sources (see e.g. [Fal03; Sol +08 ; Ull +12 ; Rud +10$]$ ), and different analytical approximations (e.g. [WK94; Neh86; Smi+01]) of buffered calcium reaction diffusion equations. One usually finds the same phenomenon over and over again: the $\left[\mathrm{Ca}^{2+}\right]$ evolution around single channels and clusters of channels live on different temporal and spatial scales and show very distinct gradients. The following separation into two categories tries to capture this distinction ${ }^{2}$.

\section{Nanodomain}

In the close vicinity (up to few hundred nanometers) of individual channels the openings and closings of the channel induce very rapid changes of steep $\mathrm{Ca}^{2+}$ profiles around the channel pore, which will be called nanodomains [TTF09]. A snapshot of these nanodomains can be seen in fig. 6.1 and is given as a schematic in fig. 6.2 A. In fig. 6.1 the peak amplitude reaches up to $80 \mu \mathrm{M}$, and was reported to exceed $100 \mu \mathrm{M}$ by others [TF04; Rud +10$]^{3}$. Despite the steep gradients of the nanodomains, the surrounding channels can still feel them. This is because clusters of channels are usually rather compact, i.e. the inter channel distance is less than $200 \mathrm{~nm}$ [DM11; Swi+99], and the total cluster size is on the order of

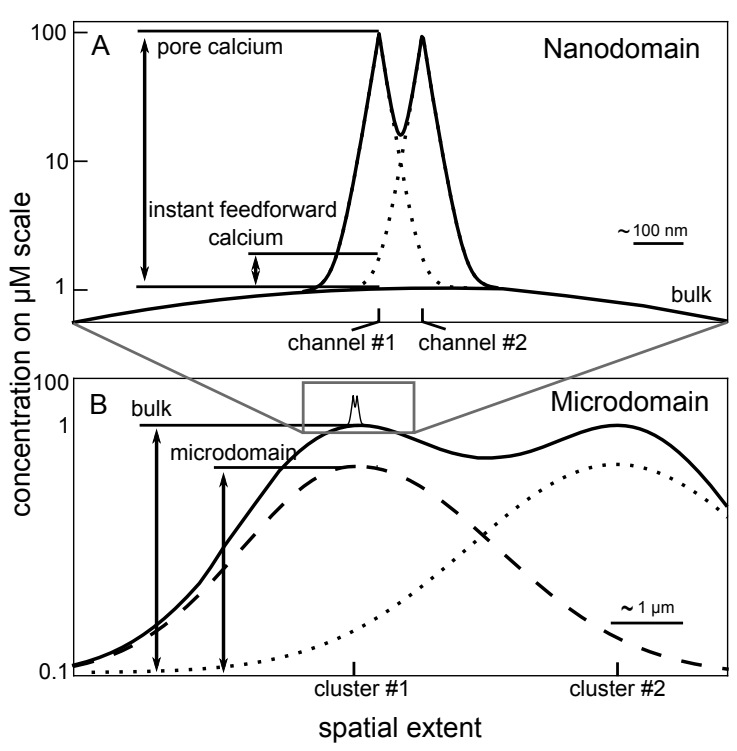

Fig. 6.2: Schematic of different calcium scales employed in the CGM.

$0.5 \mu \mathrm{m}[\mathrm{Cal}+98$; SP09; SRP06]. This means, the calcium concentration at a closed pore is dominated by the contributions of nanodomains of surrounding open channels. Figure 6.1 also depicts the rapid collapse of the nanodomains: $50 \mathrm{~ms}$ after the last channel has closed, they have disappeared completely.

Naturally, the contributions of neighboring channels' nanodomains to the concentration at a closed channel's pore is affected by various parameters like the buffering

\footnotetext{
${ }^{2}$ The naming of the two categories in this work tries to represent the spatial scales. Both names, microdomains and nanodomains, are frequently used throughout literature and their meaning might differ from source to source.

${ }^{3}$ See also section 5.1 for an explanation why the peak amplitude here was lower.
} 


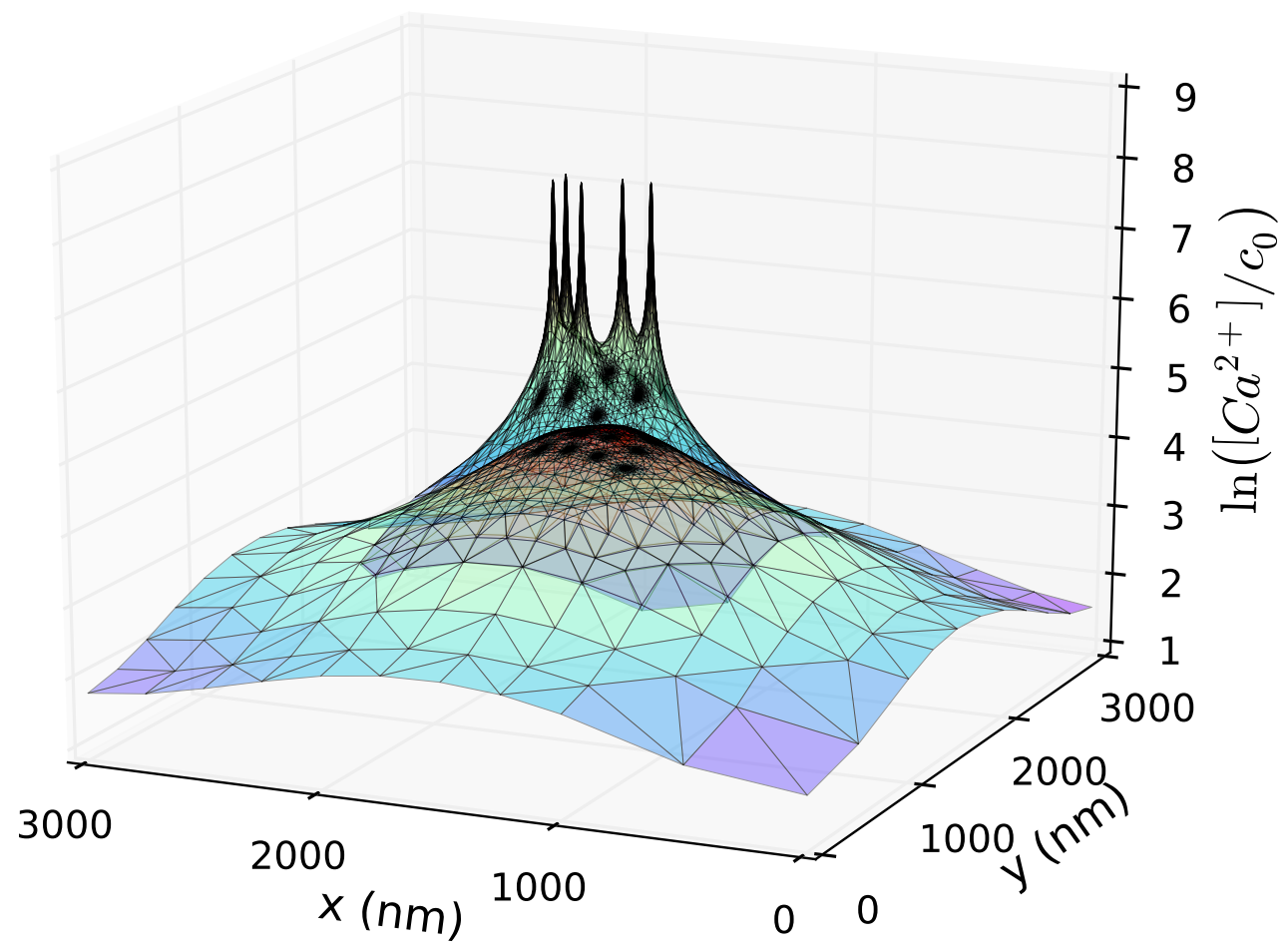

Fig. 6.1: Calcium profiles of the simulation domain from chapter 5 with nanodomains at channel pores, and microdomain, after all channels have closed. One can see that the $\mathrm{Ca}^{2+}$ concentrations ranges through 3 to 4 decades (logarithmic $\left[\mathrm{Ca}^{2+}\right]$ axis) and that the timescales of nanodomain collapse is on the order of a few milliseconds (time delta between the two snapshots: $49 \mathrm{~ms})$. The much longer time scale of the microdomain collapse can be seen in fig. 6.3 . 
settings, the inter channel distance, or the $\mathrm{Ca}^{2+}$ conductivity of the channels. Usually the contribution ranges of the order of $1 \mu \mathrm{M}$ to $10 \mu \mathrm{M}$ [RSS10]. To account for the nanodomains, the following model will make four approximations. First, a mean field approximation is installed for the superposition of nanodomains within the cluster. This means, all closed channels of a cluster feel the same nanodomain contributions, which is directly proportional to the number of open channels in the cluster. This direct proportionality is motivated by [RSS10] where it was shown that the instant feedback $c_{\text {if }}$ shows approximately a linear dependence on the number of open channels. If experiments or FEM simulations indicate a violation of this assumption, an adaption of the following model to a more accurate mean field is straightforward. Second, because of the rapid built up and collapse of the nanodomain, it is assumed that the mean field equilibrates instantaneously. Third, if a channel is open, it will only feel the peak concentration of its own nanodomain ${ }^{4}$. Fourth, because of the large spatial separation of clusters, each cluster has its own independent mean field and the mean fields of different clusters are not coupled.

\section{Microdomain}

The continuous flux of $\mathrm{Ca}^{2+}$ through the open channels accumulates in the cytosolic domain and due to diffusion and reaction with buffers, the $\left[\mathrm{Ca}^{2+}\right]$ gets smeared over a larger domain covering the whole cluster area (fig. 6.2 A). That means below the nanodomains, there exists a more coarse grained structure of the concentration field which will be called microdomain. Since the equilibrium of that structure depends on the total efflux of $\mathrm{Ca}^{2+}$ from the cluster, the microdomain slowly accumulates and decays towards its equilibrium, when channels of the cluster open or close, respectively. Naturally, when all channels are closed, the equilibrium field corresponds to the resting calcium concentration of the system. Because of the rather slow evolution of the microdomain, it can be seen as a varying baseline on top of which the nanodomain dynamics takes place. The last big difference to nanodomains, besides its slower dynamics and much larger extent, is its concentration amplitude: It usually resides in the sub $\mu \mathrm{M}$ regime.

To achieve the goal of maximal simplicity, the following model will completely neglect the spatial structure of the microdomain. Instead, the microdomain will be defined only by its amplitude which evolves according to a simple ODE. This ODE incorporates the equilibration towards the "dynamical steady state" via a dependency on the number of open channels. Finally, because of its larger spatial extent, the microdomain of a cluster may be felt by neighboring clusters.

\footnotetext{
${ }^{4}$ In fact, the same peak concentration as in section 5.1 will be used.
} 


\subsection{Model description}

While the nanodomains are essentially a feature of the calcium profile, which corresponds to an individual channel, the following equations do only account for the mean field of nanodomains $c_{\mathrm{nd}, i}$. This mean field as well as the microdomains $c_{\mathrm{md}, i}$ are cluster specific features and will be denoted with a subscript $i$ identifying the $i$-th cluster. Further, all equations only contain the fraction of open channels. This allows to use the same description for different numbers of channels per cluster without the need to rescale the other phenomenological parameter, which is useful to compare parameter sets with differently sized clusters ${ }^{5}$.

The first equation of the model describes the mean field of nanodomains within a cluster, $c_{\mathrm{nd}, i}(t)$, which is felt by all closed channels of the cluster:

$$
c_{\mathrm{nd}, i}(t)=c_{\mathrm{if}} n_{\mathrm{o}, i} .
$$

The fraction of open channels of the cluster $i$ is depicted by $n_{\mathrm{o}, i}$, and, similar as in [RSS10], all channels contribute an equal amount which is controlled by $c_{\mathrm{if}}{ }^{6}$.

The second cluster specific variable is the microdomain amplitude $c_{\mathrm{md}, i}$. As stated before, the microdomain is subject to an equilibration towards a "dynamical steady state". Here, this equilibration is incorporated via a direct proportionality of the accumulation speed to the fraction of open channels, $n_{\mathrm{o}, i}$, i.e. to the total current through all open channels of the cluster and the parameter $\gamma$ :

$$
\frac{\mathrm{d} c_{\mathrm{md}, i}}{\mathrm{~d} t}=\gamma n_{\mathrm{o}, i}-P_{\mathrm{p}} \frac{c_{\mathrm{md}, i}^{2}}{c_{\mathrm{md}, i}^{2}+K_{\mathrm{p}}^{2}}+P_{1} .
$$

In order to remove $\mathrm{Ca}^{2+}$ from the microdomain, SERCA pumps are incorporated via the second term on the right hand side. As in section 5.1 they are activated according to a Hill function. The last term $P_{1}$ is required to drive the microdomain to its resting concentration $c_{0}$ when no channels are open. It can be regarded as leakage from the ER and it compensates the current of the SERCA pumps at rest state:

$$
P_{1}=P_{\mathrm{p}} \frac{c_{\mathrm{rest}}^{2}}{c_{\mathrm{rest}}^{2}+K_{\mathrm{p}}^{2}}
$$

Finally, the coupling between clusters is mediated via the microdomains. Here, the coupling is chosen such that it does not have any feedback on the microdomain itself.

\footnotetext{
${ }^{5}$ Since noise in our model is only introduced by the MC gating of the $\operatorname{IP}_{3} \mathrm{R}$ the number of channels provides a mechanism to "scale noise", i.e. more channels correspond to less noise.

${ }^{6}$ It is assumed that the contribution $c_{\text {if }}$ does not depend on the number of open channels itself. While this is the most simple approach, one could argue that there should be some saturation effects originating from the reaction diffusion system of $\mathrm{Ca}^{2+}$ and buffers.
} 
The main reason for this choice is that it allows to compare single cluster with multi cluster simulations. Hence, a new variable per cluster, the bulk concentration $c_{\text {bulk }, i}$, is introduced as follows:

$$
c_{\mathrm{bulk}, i}=c_{\mathrm{md}, i}+\alpha \sum_{j \in S_{i}} \operatorname{Max}\left[0, c_{\mathrm{md}, j}-c_{\mathrm{md}, i}\right] .
$$

The set of neighbors of cluster $i$ is depicted as $S_{i}$ and $\alpha$ denotes the coupling strength. The dependence $\operatorname{Max}(a, b)$ is used to model the asymmetry in the coupling of clusters, where, in contrast to diffusive coupling, only a small fraction of $\mathrm{Ca}^{2+}$ released at one cluster arrives at the neighboring clusters [RS09].

Finally, one yields the $\mathrm{Ca}^{2+}$ concentration at the $g$-th channel of cluster $i$ :

$$
c_{i}^{g}(t)= \begin{cases}c_{\mathrm{open}}, & \text { if } g \text { is open } \\ c_{\mathrm{bulk}, i}+c_{\mathrm{nd}, i}, & \text { if } g \text { is closed }\end{cases}
$$

It was assumed that the cluster's local mean field from eq. (6.1) adds up linearly with the bulk contribution eq. (6.4) when the channel is closed. If the channel is open, bulk, microdomain, and mean field are neglected and the local $\left[\mathrm{Ca}^{2+}\right]$ at the channel pore is assumed to be of constant amplitude $c_{\text {open }}$. The concentration from eq. (6.5) is then

\begin{tabular}{|c|c|c|c|}
\hline Parameter & Symbol & Value & Unit \\
\hline Coupling coefficient & $\alpha$ & 0.125 & 1 \\
\hline Cytosolic resting $\mathrm{Ca}^{2+}$ & $c_{\text {rest }}$ & 0.02 & $\mu \mathrm{M}$ \\
\hline Instant. feedforward (others) & $c_{\mathrm{if}}$ & 4.8 & $\mu \mathrm{M}$ \\
\hline Instant. feedback (self) & $c_{\text {open }}$ & 150 & $\mu \mathrm{M}$ \\
\hline Channel flux & $\gamma$ & 4.8 & $\mu \mathrm{M} / \mathrm{s}$ \\
\hline Pump strength & $P_{\mathrm{p}}$ & 0.2 & $\mu \mathrm{M} / \mathrm{s}$ \\
\hline Pump diss. const. & $K_{\mathrm{p}}$ & 0.1 & $\mu \mathrm{M}$ \\
\hline \# Channels per cluster & $N$ & 16 & 1 \\
\hline \# of clusters & $M$ & 1 or $8 \times 8$ & 1 \\
\hline $\mathrm{IP}_{3}$ concentration & {$\left[\mathrm{IP}_{3}\right]$} & 2 to 100 & $\mathrm{nM}$ \\
\hline
\end{tabular}
fed into the DYK model from section 3.4 to determine the individual channels gating.

Tab. 6.1: Model parameters for eqs. (6.1)-(6.5).

Figure 6.3 shows a comparison of the model from section 5.1 with the model introduced here. In fact, many of the parameters from table 6.1 where chosen in such a way, that the CGM matches the main features of the HDM calculations. To generate 


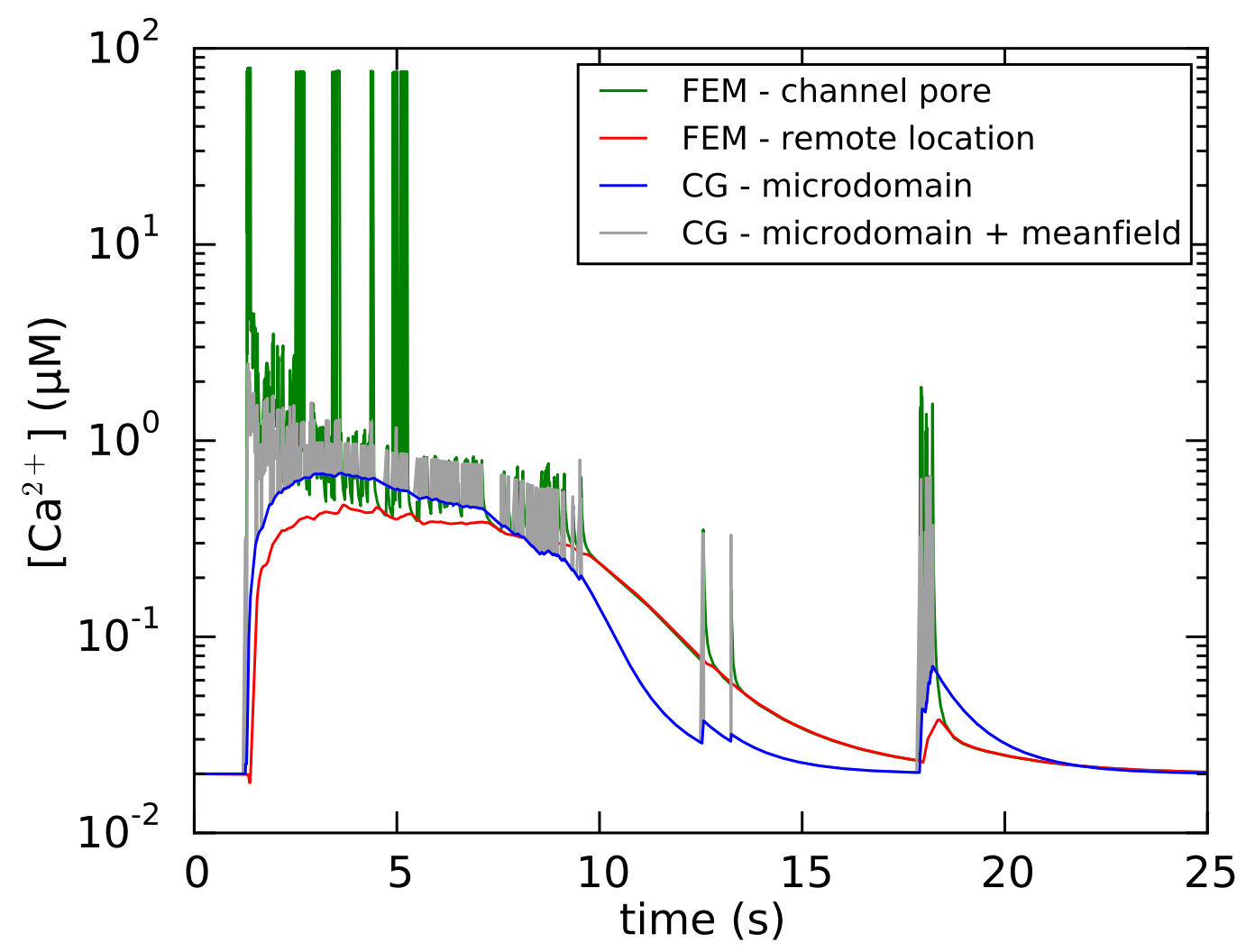

Fig. 6.3: Comparison between FEM model (green and red) and coarse grained model (gray and blue). The green line depicts the local $\left[\mathrm{Ca}^{2+}\right]$ at the $\mathrm{IP}_{3} \mathrm{R}$ pore of one arbitrary channel from the FEM simulations. One can see the huge peaks when this channel is open, which accredit for neglecting all other contributions in that case. Further, one can see the smaller rapid fluctuations induced by the nanodomains from the other channels, which obviously are substituted to some baseline that rapidly builds up during the start of the event. The red line shows the $\left[\mathrm{Ca}^{2+}\right]$ of the FEM at a remote location (distance to cluster $\sim 2 \mu \mathrm{m}$ ). The massive efflux of $\mathrm{Ca}^{2+}$ during the event leads to a substantial increase at the edge of the domain, which could potentially be felt by neighboring clusters. The blue and gray line show $c_{\mathrm{md}}$ and $c_{\mathrm{md}}+c_{\mathrm{nd}}$ of the cluster, respectively. One can see the rapid fluctuations of the nanodomains on top of the slowly evolving dynamical offset, similar as for the FEM model. 
this comparison, a special simulation with the new model and a deterministic replay of the wave from the FEM simulations (the wave at $t=115 \mathrm{~s}$ from fig. $5.2 \mathrm{G}$ ) was performed. I.e. the ODE from eq. (6.2) was integrated for a single cluster (i.e. no coupling contributions from other clusters) to obtain $c_{\text {nd }}$. Then, the corresponding total $\mathrm{Ca}^{2+}$ concentration from eq. (6.5) was calculated for this wave's specific deterministic channel gating and a closed channel $g$. If not stated otherwise the parameters above, together with the second set of parameters from table 3.1 were used to generate the results in the following sections.

In general, the phenomenological nature of the model allows for the selection of appropriate and realistic values for various cell types and situations. Since the following section does not focus on any specific cell type or experimental setup, the used parameters a thought to serve as a reasonable template. For a skeptic reader one can argue, that the chosen parameters are at least on the correct scale (see comparison with the FEM in fig. 6.3). Nonetheless, the following paragraphs provide a short discussion on the implications of the phenomenological parameters and their real world counterparts. This will facilitate the adaption of parameters for a more quantitative comparison to experiments or other highly detailed models (e.g. [Can+13; FOB00; Ruc+15]).

The impact of buffers on the phenomenological parameters is probably the most difficult to assess, since their effect depends on a multitude of real world parameters: the buffer concentration, the $\mathrm{Ca}^{2+}$ affinity, and the buffer diffusion coefficient. Plausibly, higher buffer concentrations and affinities will lead to both diminished micro- and nanodomains, i.e. lower $c_{\mathrm{if}}$, lower $c_{\mathrm{open}}$, and lower $\gamma$. A good overview of the effects of buffer on the effective diffusion coefficient of $\mathrm{Ca}^{2+}$ is given in [KS10][sec. 7.4.1]. Very likely quantitatively reasonable choices of these parameters can only be made by conducting either FEM simulations or experiments in a very well controlled environment. Alternatively, a system of coupled ODEs incorporating bound and unbound buffers in the microdomain might be a reasonable extension for a systematic investigation on the effect of buffers.

As stated before, the contributions of individual nanodomains to the mean field highly depend on the density of channels within the cluster, the ion current per channel, or the buffer capacity. Higher channel current or more dense clusters will increase the nanodomain overlap at the channel pores, and hence would be reflected by a higher $c_{\mathrm{if}}$. The same argument can be followed for the pore calcium concentration $c_{\text {open }}$, since it essentially denotes the peak amplitude of the nanodomains.

The parameter $\gamma$ will also increase with higher channel conductivity (or higher ER $\mathrm{Ca}^{2+}$ concentration). Faster effective diffusion will again lead to a slower accumulation of the microdomain and hence a diminished accumulation rate $\gamma$. 
It is known that SERCA is activated according to a Hill function with given $K_{\mathrm{p}}$, leaving $P_{\mathrm{p}}$ as undetermined parameter. Usually activation of SERCA depends on various other physiological parameters (see section 5.1 and [IKL88; Sch89; Lyt+92; Fal04]). Further, in this model, $P_{\mathrm{p}}$ also incorporates the density of SERCA making it a quite fuzzy parameter. In the following $P_{\mathrm{p}}$ is chosen such, that the decay to resting $\mathrm{Ca}^{2+}$ approximately corresponds to section 5.1.

At last, the coupling between different clusters via $\alpha$ will be diminished with increased buffer capacity, but also via faster SERCA pumps, or less "geometric confinement" of calcium ions.

\subsection{Results}

The data presented in this chapter was first published in [RR16], the computations were performed on the same infrastructure as described in section 5.3. This section will start with a concise parameter study and short comparison to the results of section 5.3 performed on the basis of a single cluster only. It will then focus on a single parameter set (table 6.1) and the coupling and synchronization of a grid of $8 \times 8$ clusters. Because simulations of this model require much less computational effort, simulation intervals were chosen much larger (up to 100000 s for the single cluster simulations). This usually results in much richer statistics than in the former chapter. Integration of the system of coupled ODEs for multiple clusters was done with the same code as in section 5.3 and is described in section 2.5.2.

\subsubsection{Event definition}

As in section 5.3.3, it is crucial that bursts of channel openings of a cluster are properly detected as collective event, because this allows to investigate coupling properties itemized by event properties. In this chapter, a slightly different approach compared to section 5.3.3 will be pursued: A CE is defined as the interval where at least one channel of the cluster is open. Further, after the last channel closing of a cluster, a threshold interval $\tau_{D}$ will account for reopenings of any channel of that cluster. If any channel reopens within $\tau_{D}$, the $\mathrm{CE}$ will be prolonged until all channels are closed again. This procedure will be repeated until no channel openings occur within $\tau_{D}$. This method obviously introduces a lower bound of $\tau_{D}$ for measured inter event intervals (IEIs). According to fig. 6.3, the timescale of microdomain decay is on the order of seconds, and hence $\tau_{D}$ is chosen as $1 \mathrm{~s}$. As in section 5.3.3 all CEs which did not exceed a duration 


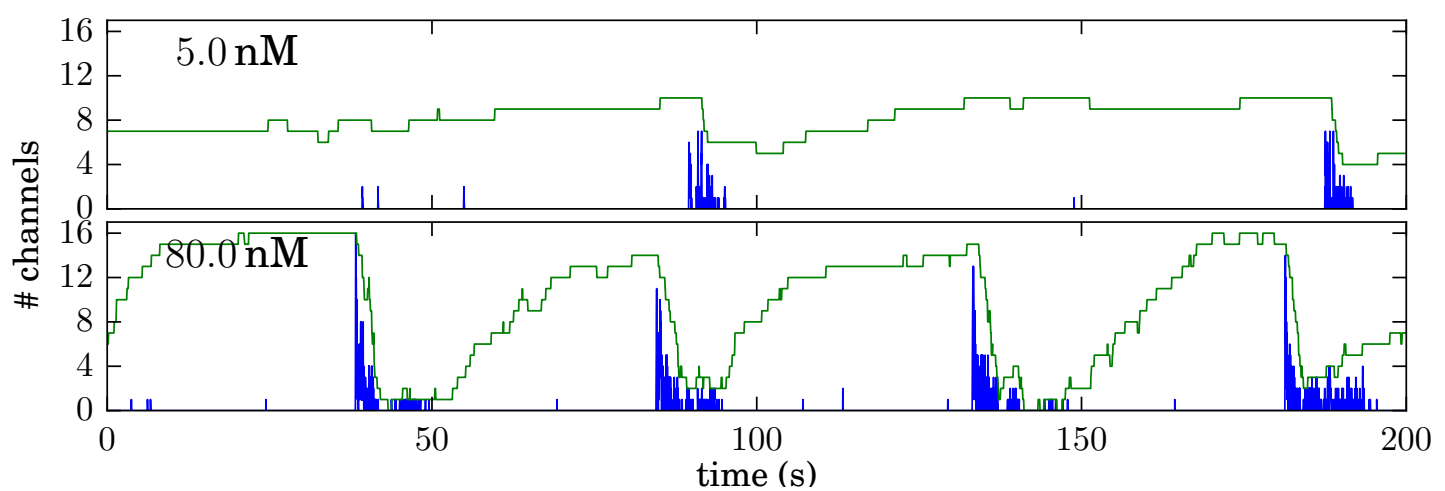

Fig. 6.4: Evolution of \# of open (blue) and activatable (green) channels for low ( $5 \mathrm{nM}$ ) and high $(80 \mathrm{nM}) \mathrm{IP}_{3}$ concentration. For $5 \mathrm{nM}$ the number of activatable channels grows only slowly. Occasionally, some small events reset the number of activatable channels, and the overall number of activatable channels only fluctuates slightly. In contrast to that, for high $\left[\mathrm{IP}_{3}\right]$ the much quicker binding of $\mathrm{IP}_{3}$ to the channels results in a fast rise of activatable channels, which frequently triggers large release events, leading to a high amplitude oscillation.

of $15 \mathrm{~ms}$ or consisted only of a single channel opening (i.e. blibs) were filtered out $^{7}$.

\subsubsection{Single cluster simulations}

Figure 6.4 shows the evolution of channel states (open and activatable) of a single cluster with 16 channels for low and high $\left[\mathrm{IP}_{3}\right]$. The other parameters are denoted in table 6.1. The definition of activatable is the same as in sections 3.4 and 5.3: a channel is considered activatable if three or more subunits have bound $\mathrm{IP}_{3}$. One can see a clear saturation curve of the \# of activatable channels for high $\left[\mathrm{IP}_{3}\right]$ going almost up to 16 channels. This fits well to the results of section 5.3.4 and especially to fig. 5.8. On the other hand, for low $\mathrm{IP}_{3}$ only minor fluctuations occur.

\section{Event lifetime and distribution of \# of activatable channels}

The transition to the oscillating behavior for higher $\mathrm{IP}_{3}$ which is depicted in fig. 6.4 also leads to interesting effects in the distribution of event lifetimes which is presented in fig. 6.5. Here, the the columns corresponds to $\left[\mathrm{IP}_{3}\right]=5 \mathrm{nM}$ and $80 \mathrm{nM}$, respectively. In the upper row, the SERCA pumps are slightly weaker than in the lower row $\left(P_{\mathrm{p}}=\right.$ $0.2 \mu \mathrm{M} / \mathrm{s}$ vs. $0.3 \mu \mathrm{M} / \mathrm{s})$. The three different lines depict three different accumulation rates $\gamma$. Like in fig. 5.5 the distribution generally becomes broader with increasing $\mathrm{IP}_{3}$

\footnotetext{
${ }^{7}$ This filtering procedure was not done in [RR16], it is performed here for better comparison to the results of section 5.3 .
} 
concentration. More interesting is the effect of the accumulation rate $\gamma$ and the rate of the SERCA pumps $P_{\mathrm{P}}$. Increasing $\gamma$ to values above $1.6 \mu \mathrm{M} / \mathrm{s}$ leads to the formation of a second peak in the distribution for high $\mathrm{IP}_{3}$ (top left: blue and black curve). The minimum between the two peaks becomes even more pronounced for slightly increased SERCA rates, given the accumulation rate $\gamma$ is high enough (bottom left: black curve). In fact, the bimodal distribution emerges as soon as the accumulation allows to build up a sufficient microdomain during the first moments of a CE. Since the SERCA efflux antagonizes the accumulation the two parameters compete for the emergence.

To check the previous assumption, whether the number of activatable channels at event start is the controlling mechanism, which leads to the emergence of the second peak in the lifetime histograms, fig. 6.6 presents the equivalent to fig. 5.6. Here the similarity to the toy model presented in fig. 5.8 is even more stunning. While for low $\mathrm{IP}_{3}$ the distribution is rather symmetric and centered around 11 activatable channels, for high $\mathrm{IP}_{3}$ it is very skewed and peaks at a fully activatable cluster. While the results from section 5.3 also show a dependency

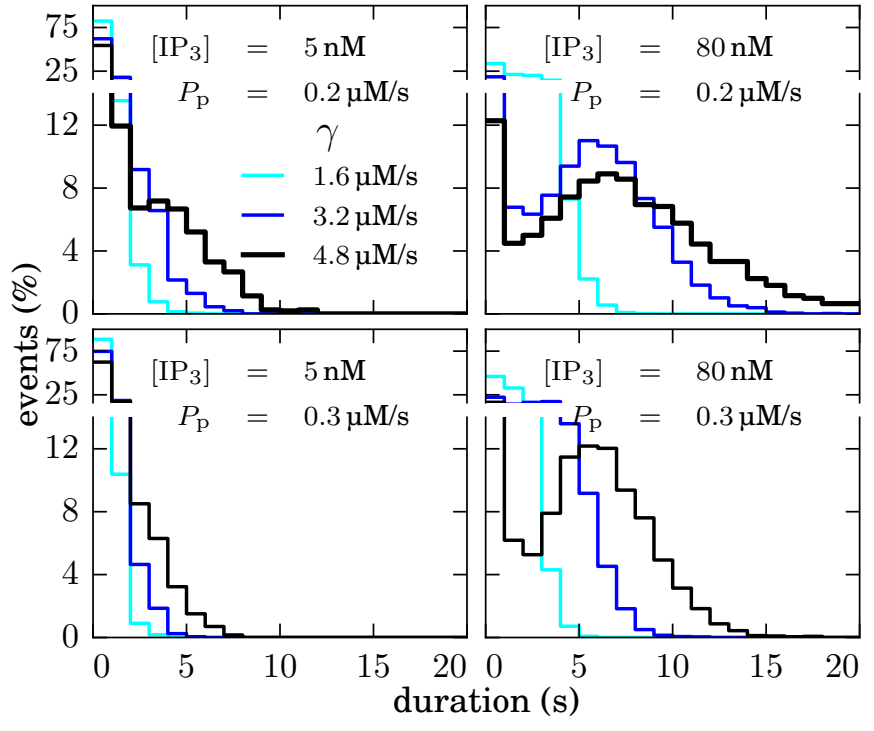

Fig. 6.5: Histograms of event lifetime of a single cluster for different SERCA strengths $P_{\mathrm{p}}$, accumulation rates $\gamma$ and $\mathrm{IP}_{3}$ concentrations. The bold black lines correspond to the parameters from table 6.1 .

of the mean \# activatable on $\left[\mathrm{IP}_{3}\right]$, here the inset of fig. 6.6 demonstrates, that this dependency is not required for forming the bimodal distribution of event lengths. Note, that the change of parameters of the DYK model between section 5.3 and this chapter has a major impact on the distributions demonstrated in figs. 5.6 and 6.6: Here, the $\mathrm{IP}_{3}$ binding rate is 10 times the former rate, which massively pronounces the effects demonstrated in fig. 5.8.

As demonstrated by [DP03], wave formation can be hampered by adding EGTA as an exogenous $\mathrm{Ca}^{2+}$ buffer. In [Joh+15] it is was shown, that the formation of nanodomains depends on SERCA uptake of $\mathrm{Ca}^{2+}$ and hence the $\mathrm{Ca}^{2+}$ load of the ER. Here, both these effects could be modeled as decrease of $\gamma$, which in turn yields matching release patterns in the simulations. 


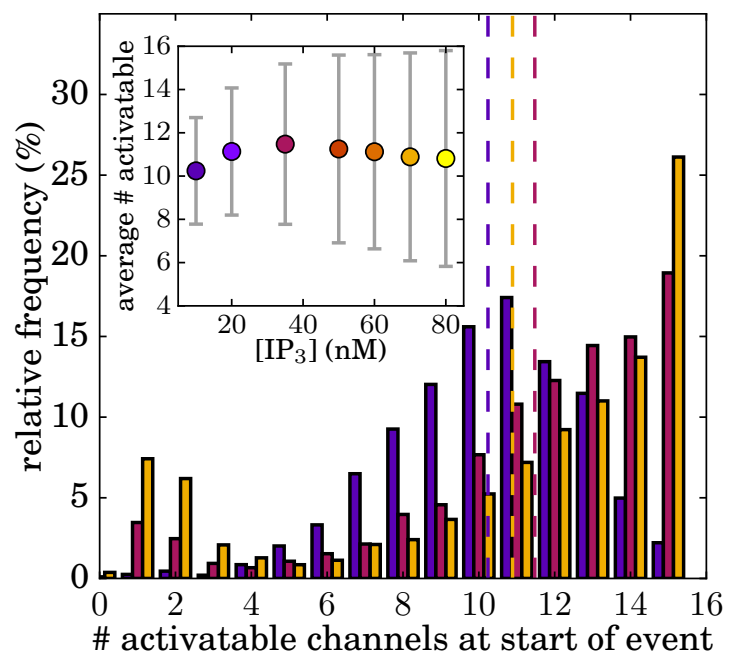

Fig. 6.6: The distribution of activatable channels at the start of an event for $10 \mathrm{nM}, 35 \mathrm{nM}$, and $70 \mathrm{nM}$. Similar as in fig. 5.6 the distribution get more skewed and moves to the right for increasing $\mathrm{IP}_{3}$ concentration. The inset demonstrates a significant increase of the variability allowing for events where a cluster is fully activatable already for intermediate $\mathrm{IP}_{3}$.

\section{Event lifetime correlates with dynamics of \# activatable}

As in section 5.3 the next step is to check the correlation of event lifetime with the number of activatable channels. Figure 6.7 shows the plot corresponding to the former results in fig. 5.7. Note, that in fig. 5.7 the axis only goes up to 12 channels, whereas here it covers the full range up to 16 channels. This is again due to the fact, that the $\mathrm{IP}_{3}$ binding rate was increased by a factor of 10 yielding much higher numbers of activatable channels throughout all simulations.

As can be seen from fig. 6.7 the event durations steadily increase with the number of activatable channels at event start starting from around 6 channels until up to a fully activatable cluster. This is even true for the lowest $\mathrm{IP}_{3}$ concentration depicted in fig. 6.7. In the case of a prolonged event, $\mathrm{IP}_{3}$ rebinding becomes more relevant for higher $\mathrm{IP}_{3}$ concentrations, which leads to higher average durations for higher $\mathrm{IP}_{3}$ concentrations on the right hand side of fig. $6.7 \mathrm{~A}$. The rise of durations for less than 5 activatable channels on the left side of fig. $6.7 \mathrm{~A}$ can be attributed to the $\mathrm{CE}$ definition: if the $\mathrm{CE}$ definition fails and splits a burst of channel openings into several individual CEs, those events will have a rather long duration but less activatable channels at start. Therefore, they distort the distribution presented in fig. 6.7. Interestingly, the large amount of gathered statistics and the faster $\mathrm{IP}_{3}$ binding rate shows, that event for low $\mathrm{IP}_{3}$ concentration the long release events may occur. 

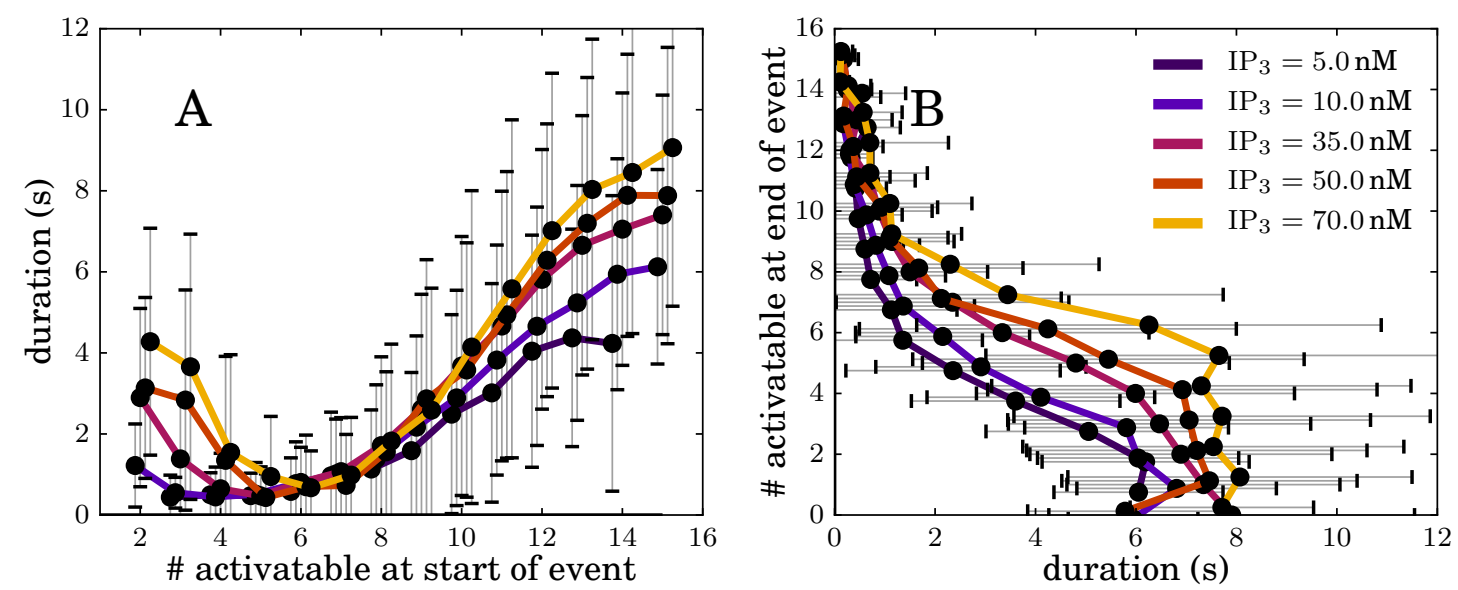

Fig. 6.7: A: Similar as demonstrated in fig. 5.7, the number of activatable channels at the start of an event controls the lifetime distribution of the event. Many activatable channels at start will likely result in a prolonged release. B: Long lasting release leads to a significant inactivation of the cluster.

The right hand side of fig. 6.7 leads to the same conclusions as in the previous chapter: long lasting release will result in a essentially inactivated cluster, and this inactivation also provides the necessary termination mechanism.

The bimodal distribution of fig. 6.5 becomes even more apparent, when one itemizes the events by duration and the effective number of channels which get deactivated during the event ${ }^{8}$. The resulting $2 \mathrm{D}$ histogram is presented in fig. 6.8. With increasing $\mathrm{IP}_{3}$ the second class of long lasting events where many channels lose $\mathrm{IP}_{3}$ appears in the top right corner. While there is some overlap in the duration between those two classes in the area of about $4 \mathrm{~s}$, the two dimensional distribution shows a even better separation into the two different types than fig. 6.5.

\subsubsection{Grid of $8 \times 8$ clusters}

The hypothesis that the two different observed release patterns have different coupling properties cannot be further investigated unless one couples multiple clusters together. Therefore in this section the simulated system is extended to include a periodic grid of $8 \times 8$ clusters, where each cluster is coupled to its four nearest neighbors. Since one can assume that a cleaner separation of the events into the two populations will also show a stronger effect on the synchronization of global release events, the parameters are chosen

\footnotetext{
${ }^{8}$ Effective in the sense: number activatable at start minus number activatable at the end of the event.
} 


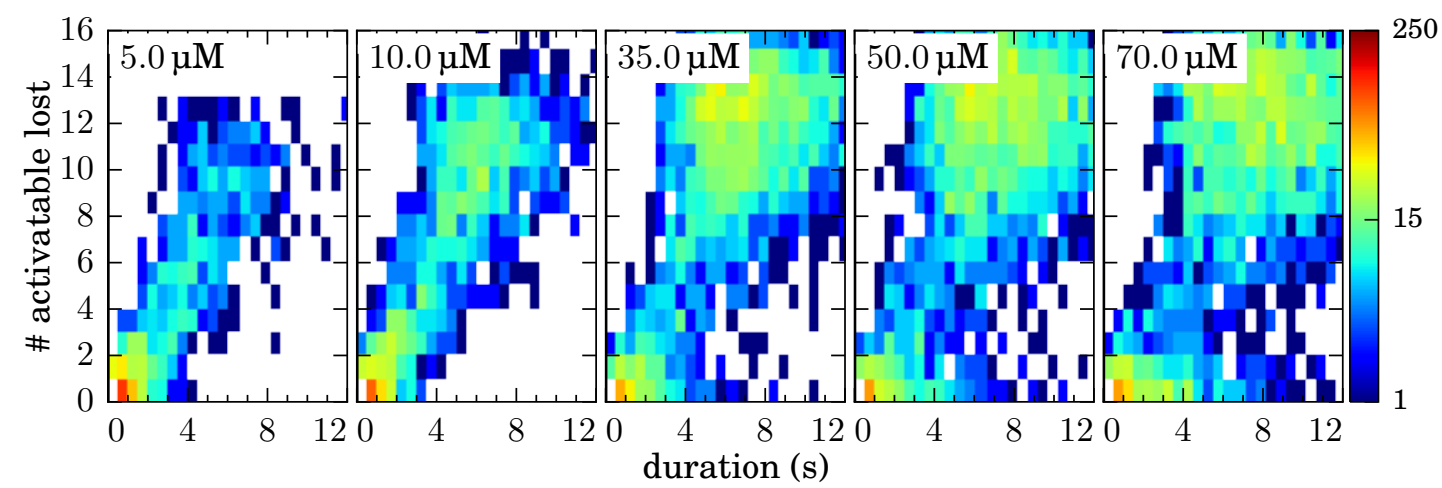

Fig. 6.8: Histogram over event duration and \# of activatable channels lost during the event for different $\mathrm{IP}_{3}$ concentrations. The color denotes the number of events in the respective bin. Events from the bottom left bin (duration less than $0.5 \mathrm{~s}$ and no lost activatable channels) were excluded.

such as denoted by the thick black line in fig. 6.5. The grid forms a two dimensional torus to reduce the impact of the finite size of the system and to facilitate data analysis and implementation. Since the 64 fold amount of channels in the system also results in about the same factor for the required computation time, the simulation times decreased from $100000 \mathrm{~s}$ in the previous sections to $\sim 20000 \mathrm{~s}$ for low $\mathrm{IP}_{3}$ concentrations and to $\sim 5000$ s for higher $\mathrm{IP}_{3}$.

A representative excerpt of the data of these simulations, once for low and once for high $\mathrm{IP}_{3}$, is presented in fig. 6.9. Similar as in visualizations of some experimental methods, a cut through the grid is used to resolve the spatio-temporal patterns, further the overall average $\left[\mathrm{Ca}^{2+}\right]$ and the percentage of activatable channels are depicted. For large $\mathrm{IP}_{3}$ several highly correlated release events travel through the system, whereas for low $\mathrm{IP}_{3}$ only some minor fluctuation occur, which remain uncorrelated. This difference is also very well reflected in the pronounced oscillations in the two other observed parameters for high $\mathrm{IP}_{3}$. The time average number of activatable channels is about identical for both simulations, which seems counter intuitive. However, a similar effect was already observed in fig. 5.6, and the same argument applies again: it is not necessarily the average number of activatable channels, but the width of the distribution which separates the dynamics for different $\mathrm{IP}_{3}$.

The snapshots in the center of fig. 6.9 resolve one of the propagating wave fronts. Obviously the two dimensional propagation is very irregular, which can be caused by a magnitude of reasons. Probably most important is the relatively small size of only eight clusters in each direction. However, the model might also show a similar feature as the turbulent backfiring reported for simulations of a larger grid of $\mathrm{IP}_{3} \mathrm{R}$ [FTL00]. 


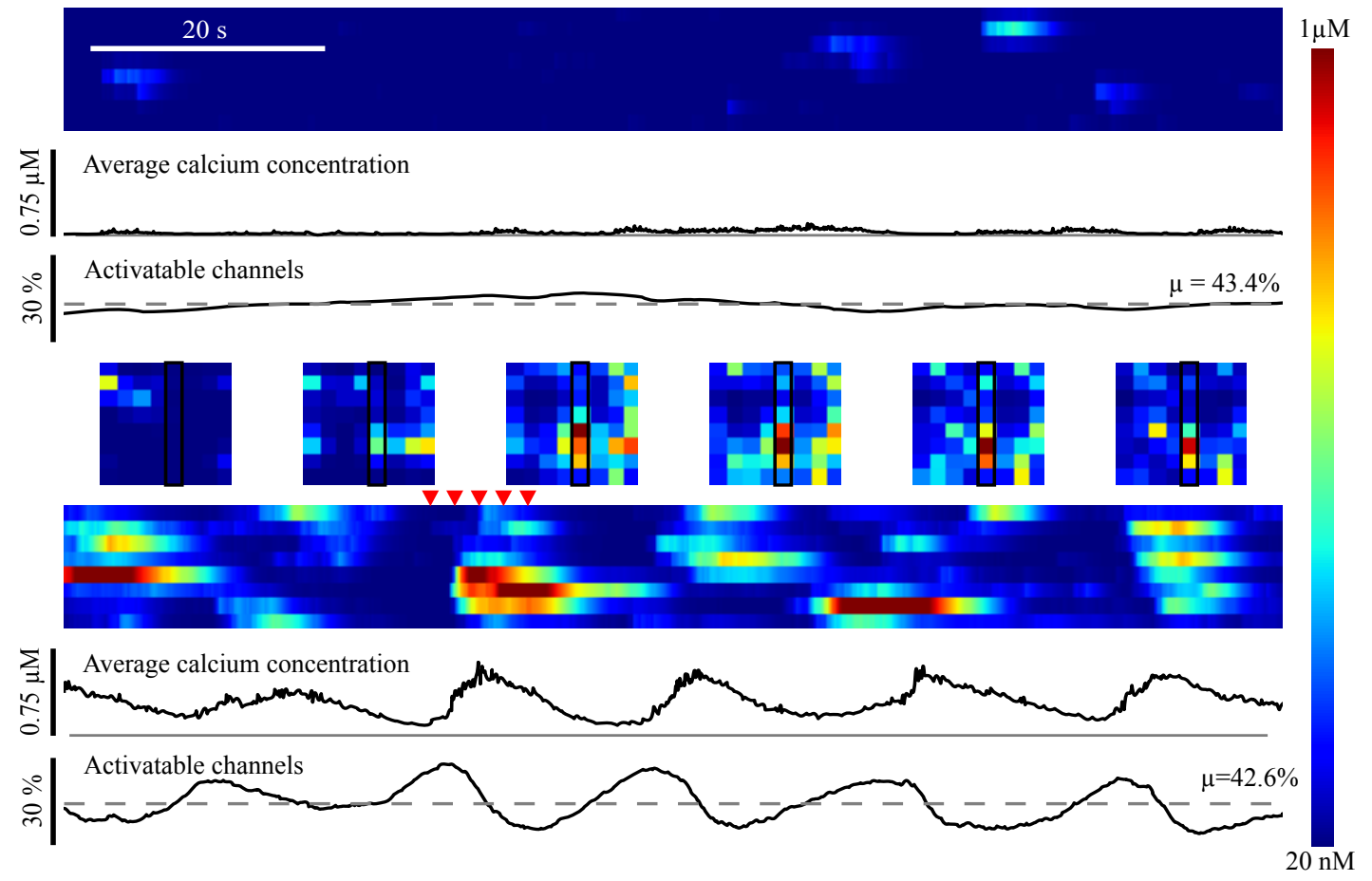

Fig. 6.9: Exemplary time series for low $(10 \mathrm{nM}$, top $)$ and high $\left(80 \mathrm{nM}\right.$, bottom) $\mathrm{IP}_{3}$ concentration. For each simulation, a line scan running through one column of the cluster, the average $\mathrm{Ca}^{2+}$ concentration over all clusters, and the total percentage of the activatable channels is depicted from top to bottom, respectively. Both, the color coded concentrations as well as the average concentration are evaluated from eq. (6.4) and do not include any nanodomain contributions. The middle row shows snapshots of the high $\mathrm{IP}_{3}$ simulation as indicated by the red arrows. The column of the line scan is depicted as black frame around the fifth column. The gray lines show the resting calcium concentration $c_{0}$ and the time average over the activatable channels, respectively. 
Finally, the most important aspect of fig. 6.9 is the high amplitude oscillation of the number of activatable channels in the case of high $\mathrm{IP}_{3}$. It shows that the $\mathrm{IP}_{3}$ unbinding during prolonged release serves as the down regulating mechanism which operates on a much slower timescale than regular inhibitory $\mathrm{Ca}^{2+}$ binding. As explained in [GS08] puffs can terminated by inhibition in two distinctive ways, either by a quasi permanent reduction of the active channels, or by rapid inhibition during the upstart of the event. In our case, one could argue that the $\mathrm{IP}_{3}$ unbinding provides sort of a long term inhibition of the first form.

\section{Event synchronization}

In section 4.4.1 a measurement called event synchronization (ES) was already introduced, which will be used here. The quantity $J_{n m}^{\mu \nu}$ denotes whether the $\mu$ th event of cluster $n$ is coupled to the $\nu$ th event of cluster $m$. Since every cluster is only coupled to its four nearest neighbors, it makes sense to calculate the number of synchronized (neighboring) clusters of the $\nu$ th event of a cluster $n$. This number is given as:

$J_{n}^{\mu}=\sum_{m \in S_{n}} \begin{cases}1, & \text { if } \sum_{\nu} J_{n m}^{\mu \nu}>0 \\ 0, & \text { else. }\end{cases}$

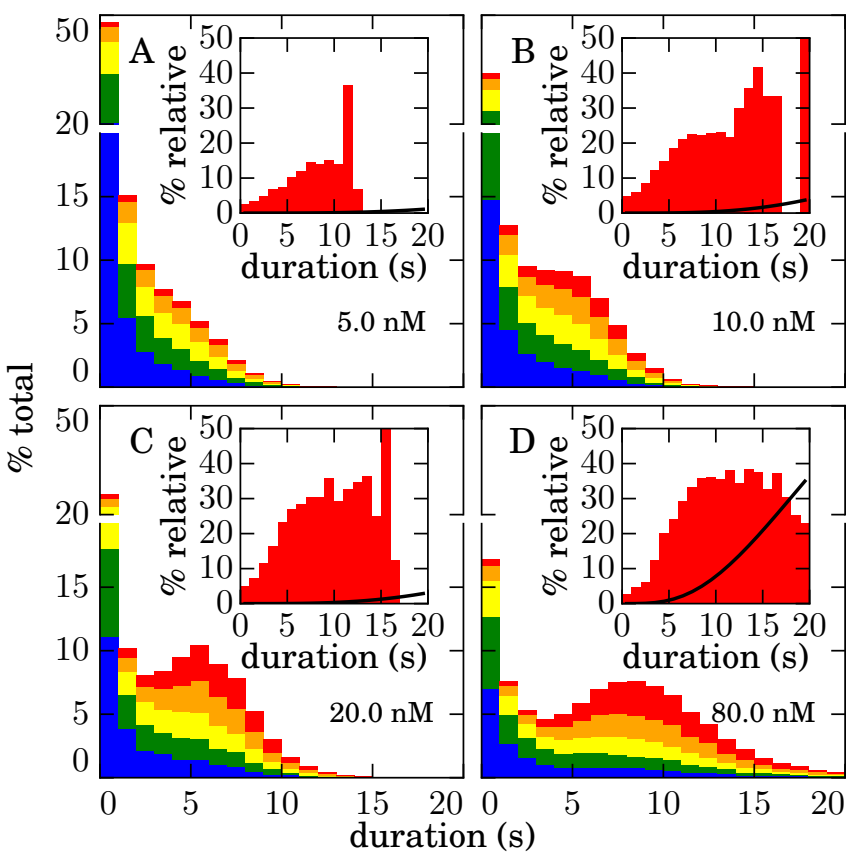

Here, the inner sum $\sum_{\nu} J_{n m}^{\mu \nu}$ will Fig. 6.10: Event length distribution for coupled clusters identify if there are any coupled events in the respective neighbor $\nu$, and the outer sum over the set for different $\left[\mathrm{IP}_{3}\right]$. The color decodes the number of synchronized clusters from eq. (6.6). The insets show relative fraction of events where $J_{n}^{\nu}=4$.

$S_{n}$ of neighbors of $n$ will count the number of coupled neighbors, i.e. $0 \leq J_{n}^{\mu} \leq 4$. Since the number of synchronized clusters can be seen as a property of a CE (it has an index $\mu$ ) one can investigate correlations between event lifetime and $J_{n}^{\mu}$. Figure 6.10 shows the event lifetime distribution similar as fig. 6.5, but with the additional information 
about the number of synchronized clusters ${ }^{9}$. Just as in the single cluster case, increasing $\left[\mathrm{IP}_{3}\right]$ results in an increase of the average event lifetime, and the distribution becomes bimodal for $\left[\mathrm{IP}_{3}\right]$ somewhere between $10 \mathrm{nM}$ and $20 \mathrm{nM}$.

The more interesting aspect of fig. 6.10 is its color coding of the number of synchronized cluster ranging from none (blue) to all four neighbors (red). As one can see, the emergent additional peak in the distribution is formed by events which are coupled to the neighboring clusters. In fact, the majority of events having a lifetime of $1 \mathrm{~s}$ or more is synchronized with at least one neighbor.

In contrast to the main figure, the vertical axis of the inset of fig. 6.10 is normalized not to the total number of events, but to the height of the respective bin in the main plot, i.e. the fraction of events having four coupled clusters given a certain event length. For all concentrations of $\mathrm{IP}_{3}$ long events have a higher chance to trigger the neighboring clusters. Between $5 \mathrm{nM}$ and $20 \mathrm{nM}$, which is approximately the range where the bimodal distribution emerges, this effect gets especially pronounced meaning that the event length alone is not the only cause for a tighter coupling.

One may argue, that it is not surprising that longer events are more likely to be synchronized with the neighboring clusters due the definition of event synchronization: even if clusters were completely isolated, longer events would always have a larger likelihood to be synchronized, since $\sum_{\nu} J_{n m}^{\mu \nu}$ in eq. (6.6) will grow together with the length of the event $\mu$ given an independent event rate $\rho$ of the clusters. With these assumptions the probability of having $k$ coupled clusters for an event of length $t$ is given by the binomial distribution:

$$
P(k, \rho, t)=\left(\begin{array}{l}
n \\
k
\end{array}\right) p^{k}(1-p)^{(n-k)} \text { with } n=4 \text { and } p=1-\exp [-\rho t] .
$$

The black line in the inset of fig. 6.10 depicts eq. (6.7) for $k=4$ and $\rho$ chosen as the overall average event rate for given $\left[\mathrm{IP}_{3}\right]$. As one can see, the observed frequency of tightly coupled events heavily exceeds the above approximation.

\section{Complex coherence function}

The complex coherence function $(\mathrm{CCF})$ as defined in section 4.4.2 provides another mean to quantify synchrony between time series. Here, the time series which will be investigated are the microdomain $\mathrm{Ca}^{2+}$ concentrations of all pairs of clusters, i.e. all pairs of $c_{\mathrm{md}, i}$ and $c_{\mathrm{md}, j}$. First, these time series were corrected to have zero mean over time. Then, the unbiased MSC as well as the phase of the SCF was calculated for

\footnotetext{
${ }^{9}$ Note the different scaling compared to [RR16]. This is due to the different event filtering as explained in section 6.2.1.
} 


\begin{tabular}{lcc}
\hline Window type & Hanning & eq. $(4.6)$ \\
Time resolution & $\Delta t$ & $2 \mathrm{~s}$ \\
Sampling window size & $L \Delta t$ & $500 \mathrm{~s}$ \\
Window overlap & $D$ & 0.5 \\
\hline
\end{tabular}

Tab. 6.2: Parameters used for the estimation of the CCF.
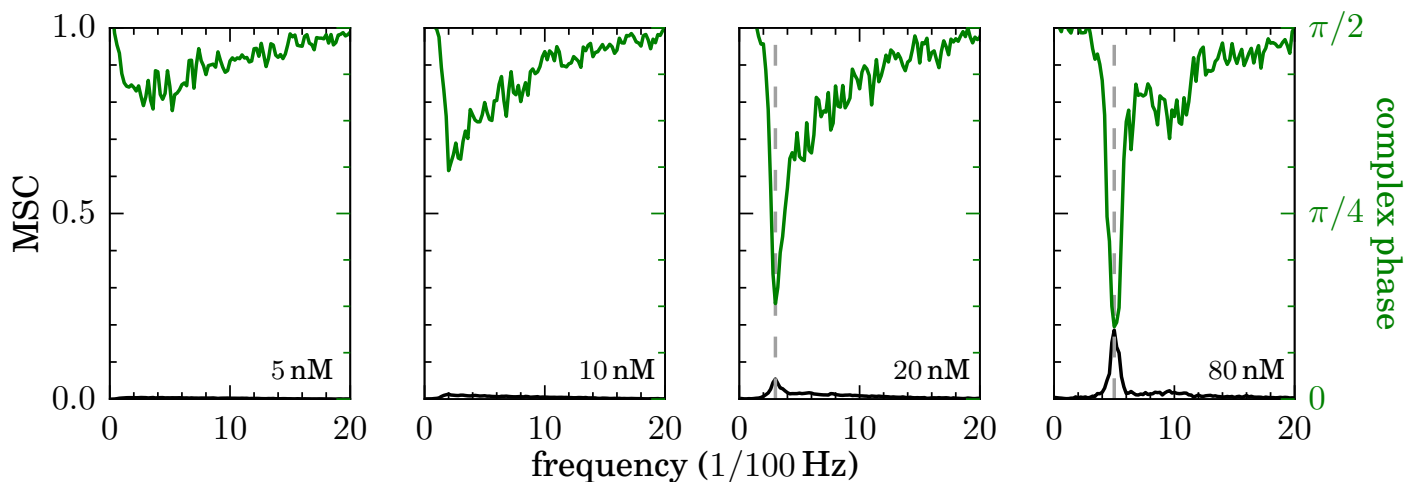

Fig. 6.12: Magnitude squared coherence (MSC) in black, and average phase shift (green) between all pairs of $c_{\mathrm{md}, i}$ for different [ $\left.\mathrm{IP}_{3}\right]$. The sampling parameters are given in table 6.2. The gray dashed line marks $f_{\text {peak }}$, the frequency of the peak in the MSC, and is omitted if MSC $\leq 0.05$. The number of samples ranges from $S=150$ for $5 \mathrm{nM}$ down to $S=23$ for $80 \mathrm{nM}$.

different concentrations of $\mathrm{IP}_{3}$ according to eqs. (4.8)-(4.10). The sampling parameters for the estimation of the $\mathrm{CCF}$ are given in table 6.2.

Figure 6.12 shows the average MSC and phase shift for different $\mathrm{IP}_{3}$ concentrations. $\mathrm{As}_{\mathrm{IP}} \mathrm{IP}_{3}$ rises, a peak in the coherence emerges (black line) meaning that the clusters' oscillations align in this frequency band. In the same frequency region, the average phase lag drops to a minimum, indicating a strong overall coupling between the clusters. Further, the peaks in the coherence shift to higher frequencies with increasing $\left[\mathrm{IP}_{3}\right]$, as will be analyzed further in fig. 6.11.

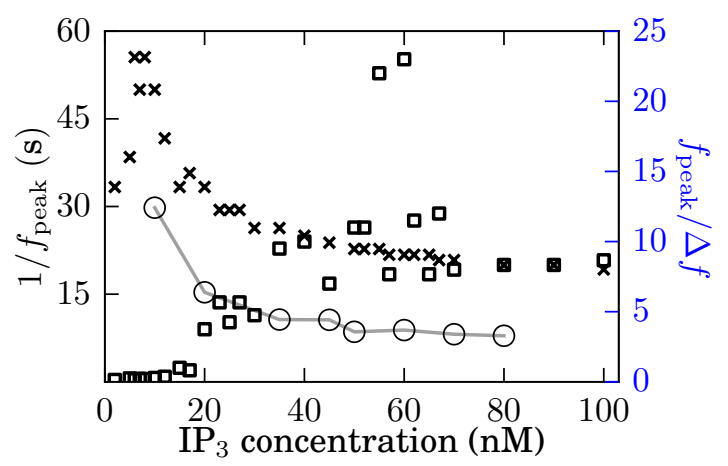

Fig. 6.11: With increasing $\mathrm{IP}_{3}$ the peak of the MSC shifts to higher frequencies (black, left axis). There appears to be a maximum in the coherence in the area of $\sim 60 \mathrm{nM}$ (blue, right

In fig. 6.11 the black markers (left axis) denote the inverse of the peak po- axis). IWIs from fig. 5.11 are showed in gray. sition $f_{\text {peak }}$ as a function of the $\mathrm{IP}_{3}$ concentration. Further, the full width at half maximum (FWHM), denoted as $\Delta f$, was calculated for all peaks of the MSC. The 
fraction $f_{\text {peak }} / \Delta f$ is depicted in blue on the right axis of fig. $6.11^{10}$.

Upon the emergence of the peak in the MSC, the position of this peak can also be used as definition for an IWI. In general, the peak is approximately at the same position as the peak in the average power spectra of the calcium concentrations (data not showed). This explains, why the trend to higher IWIs works surprisingly well, even for small $\left[\mathrm{IP}_{3}\right]$. Still, at the very low end of the spectrum, the peak of the MSC cannot be resolved from noise anymore, and the determination of the IWI fails.

Obviously, the peaks in the MSC as well as the diminished phase lags are a clear indicator for synchronization of the whole system. However, since both, global synchronization, as well as propagating waves would lead to a peak in the coherence, the underlying release pattern cannot be deduced. While there is no a priori reason for global synchronization of spatially embedded excitable systems, this effect has been reported for e.g. FitzHugh-Nagumo elements [ZKH01] as well as in intracellular $\mathrm{Ca}^{2+}$ dynamics [CT03] and is called array-enhanced coherence resonance (AECR). In systems that exhibit AECR one can tune the noise parameter such that propagating waves turn into globally synchronized oscillations. In general AECR can be detected by plotting a measure of coherence against the noise controlling parameter, which in case of AECR shows a clear peak for a certain noise value. With this in mind, one can argue, that the $\mathrm{IP}_{3}$ concentration here serves as such a noise controlling parameter, and the resonance effect is depicted by the peak of $f_{\text {peak }} / \Delta f$ in fig. 6.11 .

\subsubsection{Phaselag distributions between pairs of clusters}

To solve the question whether the synchronization between clusters is a global, or a local one. I.e. whether propagating waves may transform into globally synchronized oscillations for certain levels of $\mathrm{IP}_{3}$, this section presents distributions of phase lags between pairs of clusters. Since the phase lag is given as the complex argument of the SCF and hence is a function of frequency, a dominant phase lag $\Delta \Phi_{\mathrm{d}}^{i, j}$ between pairs of clusters $i$ and $j$ is defined, as the phase lag at maximum coherence:

$$
\Delta \Phi_{\mathrm{d}}^{i j}=\arg \left[\hat{C}_{i j}\left(f_{\text {peak }}\right)\right] \text { with } f_{\text {peak }}=\left\{f \mid \operatorname{MSC}_{i j}(f)=\min _{f^{\prime}} \operatorname{MSC}_{i j}\left(f^{\prime}\right)\right\}
$$

Figure 6.13 shows the distribution of phase lags for the different $\mathrm{IP}_{3}$ values which were simulated. As one can see from the figure a peak forms for increasing $\mathrm{IP}_{3}$ concentration, which is most pronounced and narrow for values around $50 \mathrm{nM}$ to $60 \mathrm{nM}$.

\footnotetext{
${ }^{10}$ Sometimes the fraction $f_{\text {peak }} / \Delta f$ is called quality or $Q$ factor. However, here the factor is not related to the power spectrum of a single oscillator, but to the coherence function.
} 


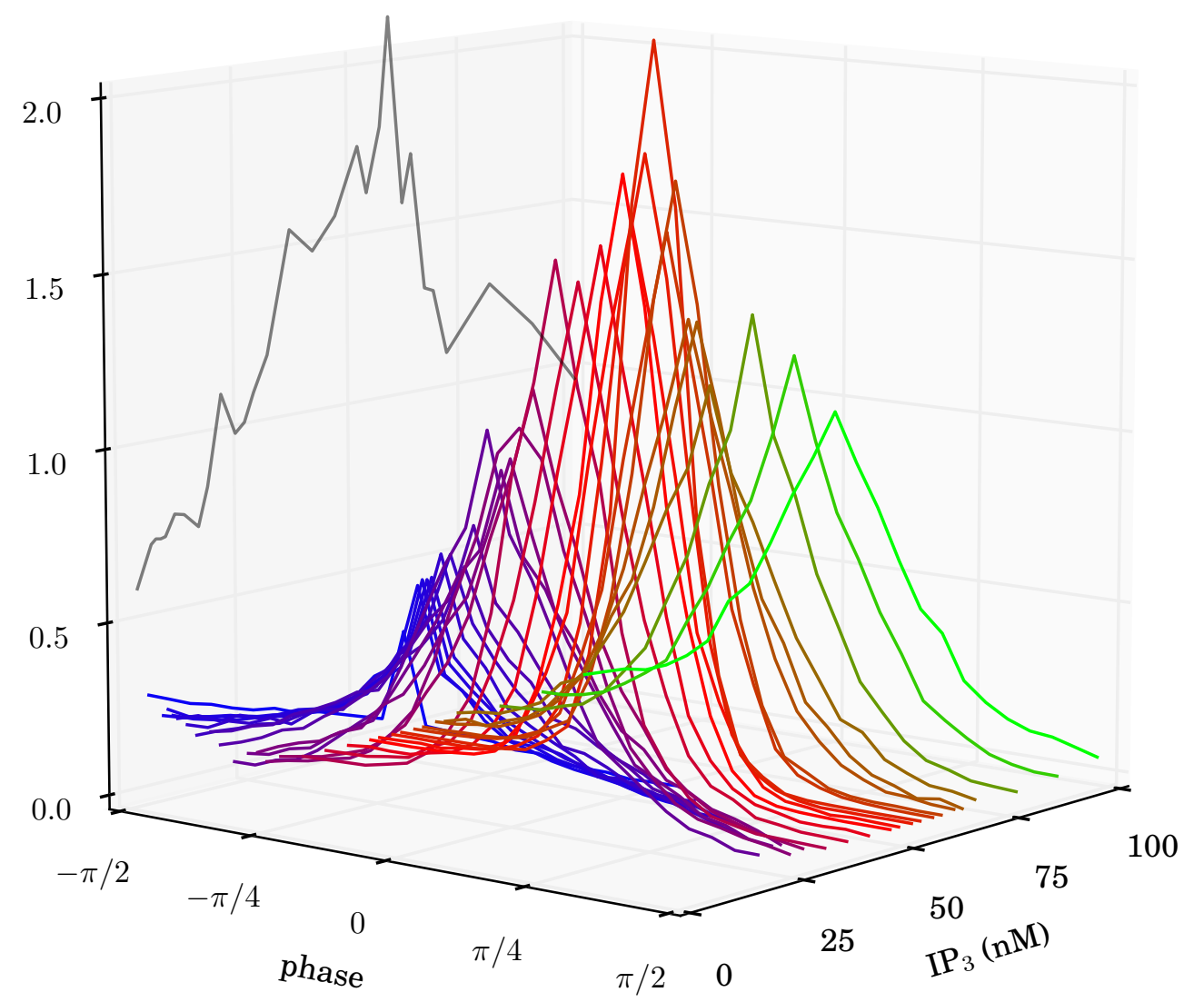

Fig. 6.13: The distribution of phase lags between all pairs of clusters as described by eq. (6.8). Since all pairs are accounted twice, the distribution if fully symmetric. For low $\mathrm{IP}_{3}$ the distribution is almost uniform with a small peak at zero phase lag. For increasing $\mathrm{IP}_{3}$ the distribution gets higher and narrower, reaching a peak at around $50 \mathrm{nM}$ to $60 \mathrm{nM}$. Then for higher $\mathrm{IP}_{3}$ concentration the distribution again smears out to a broad peak around the center. The gray line represents the ridge of the peaks, projected into to the rear plane. 
The position of the peak coincides with the extremal values of $f_{\text {peak }} / \Delta f$ presented in fig. 6.11 and nicely supports the conclusions from the previous section.

A similar histogram as presented in fig. 6.13, but only counting neighboring pairs of clusters is presented in fig. 6.14. Since there are only $64 \times 4 / 2$ individual pairs of neighbors, the sampling size does not allow to identify local minima in the distribution as one would expect for propagating waves. Hence, to decide without doubt, whether the synchronization is local, or global, a larger grid with more neighbors pairs needs to be considered.

\subsection{Discussion}

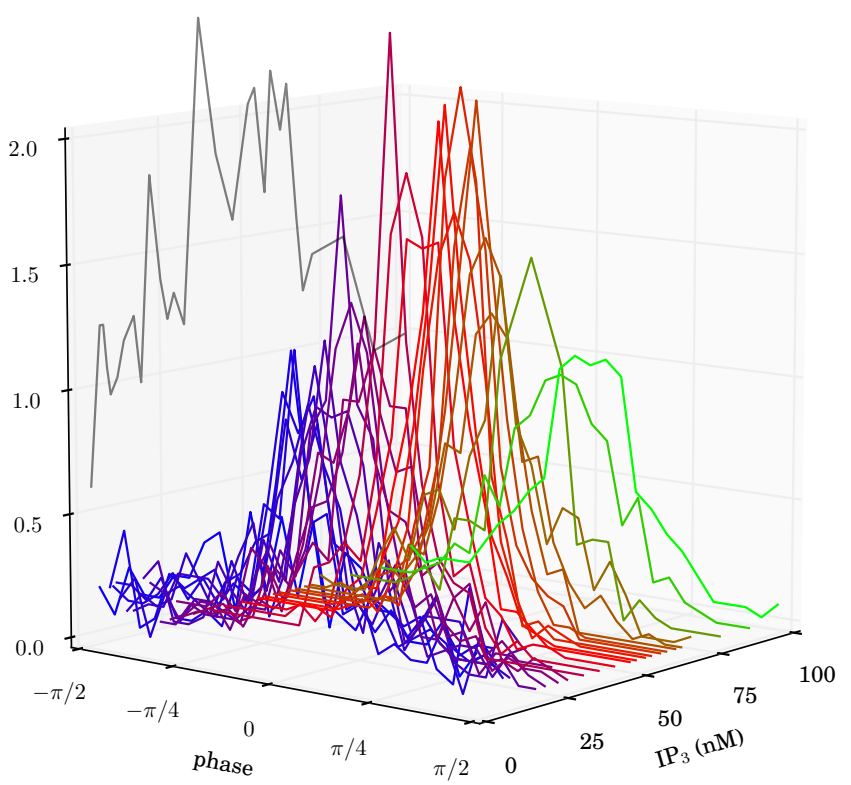

Fig. 6.14: Phase lag distribution for pairs of neighboring clusters.

This chapter demonstrated how slow scale $\mathrm{Ca}^{2+}$ release events can synchronize clusters of $\mathrm{IP}_{3} \mathrm{R}$ for increasing concentration of $\mathrm{IP}_{3}$. While release on the slow synchronized time scales is highly regular, the microscopic patterns of individual clusters still involve random puffs which are interspersed with the macroscopic oscillations. The design of the model leads to the intrinsic decoupling of small release events since they cannot accumulate sufficient amounts of $\mathrm{Ca}^{2+}$ to overcome the diffusion barrier to adjacent clusters. Therefore it gives a possible explanation for the different release time scales of puffs and waves as well as their synchronization properties.

Similarly to the previous chapter 5 the termination mechanism is provided by unbinding of $\mathrm{IP}_{3}$ from the channels, which essentially can be seen as a modulation of the cluster's excitability. Here this effect is even more important, because the positive feedback of CICR between coupled clusters leads to an overall increase of activity. It turns out that unbinding of $\mathrm{IP}_{3}$ provides an effective negative feedback counterpart which sufficiently down regulates $\mathrm{IP}_{3} \mathrm{R}$ activity (fig. 6.9). While the overall deactivation of all $\mathrm{IP}_{3} \mathrm{R}$ due to unbinding of $\mathrm{IP}_{3}$ is never complete, the amplitude of oscillation in fig. 6.9 
is still significant. More important fig. 6.7 shows that long release events just leave a cluster in an deactivated state, just as demonstrated in chapter 5. The present chapter therefore provides more evidence for the hypothesis, that $\mathrm{IP}_{3}$ unbinding may serve as the key mechanism to modulate release between puffs and waves or global oscillations.

One intriguing side effect of the $\mathrm{IP}_{3}$ unbinding during long lasting release events is the contribution of the liberated $\mathrm{IP}_{3}$ molecules to the concentration of free $\left[\mathrm{IP}_{3}\right]$, which hence shows an oscillation with the same frequency as the waves. With the above simulations and some assumptions on the cytosolic value and the density of $\mathrm{IP}_{3} \mathrm{R}$, one can approximate the amplitude of these oscillations: taking the data from fig. $6.8(70 \mathrm{nM})$ a cluster unbinds up to 15 to $30 \mathrm{IP}_{3}$ molecules ${ }^{11}$. This would result in an amplitude of oscillation for the free $\left[\mathrm{IP}_{3}\right]$ of about $1 \mathrm{nM}$ to $2 \mathrm{nM}$ if one assumes a per cluster volume of $\sim 3 \mu^{3}$. Obviously this amplitude is rather small compared to the $70 \mathrm{nM}$ of baseline concentration, and will likely not be verifiable by experiments. On the other side, the fraction of amplitude heavily depends on the $\mathrm{IP}_{3} \mathrm{R}$ density. Considering the reticular structure of the ER there may be significantly more clusters within a volume of $3 \mathrm{\mu m}^{3}$ and the fraction of amplitude to baseline could easily reach $25 \%$ to $50 \%$ as reported in e.g. [Tan+09]. Unbinding of $\mathrm{IP}_{3}$ therefore provides an explanation to passive $\mathrm{IP}_{3}$ oscillations which follow the $\mathrm{Ca}^{2+}$ oscillations, which were reported e.g. in $[$ Tan+09; Gas+14].

Coherence resonance effects for $\mathrm{IP}_{3} \mathrm{R}$ clusters have been reported before, both for a single and a grid of clusters [SJ02; SJ03]. Here, it was demonstrated, that such a phase locking between individual clusters can also emerge for intermediate $\mathrm{IP}_{3}$ concentrations, and likely breaks down for to high excitation levels. The irregular waves observed in fig. 6.9 match well to what was reported in experiments [Niv +13$]$. However, the cyclic boundary conditions, together with the rather small system size of only $8 \times 8$ clusters, prohibit a systematic investigation into the propagation aspect of the release. While one can see some clear wave fronts in fig. 6.9, the distribution of phase lags of neighboring clusters does not allow to identify a peak at nonzero phase lag which would correspond to a finite propagation speed.

\footnotetext{
${ }^{11}$ Some channels require to unbind two $\mathrm{IP}_{3}$ to become deactivated.
} 


\section{Chapter 7}

\section{Discussion}

In this work, the feedback between $\mathrm{Ca}^{2+}$ and clusters of $\mathrm{IP}_{3} \mathrm{R}$ was studied for a specific discrete state gating model of the $\mathrm{IP}_{3} \mathrm{R}$, the DeYoung-Keizer (DYK) model, and two different modeling approaches for intracellular $\mathrm{Ca}^{2+}$ distributions. The high detail model (HDM), which was used throughout chapter 5 , is capable of dealing with spatial scales on the order of individual channels, up to $\mathrm{IP}_{3} \mathrm{R}$ cluster sizes. The level of detail of this models is probably only exceeded by molecular dynamics (MD) simulations, which are not yet able to deal with such large simulation domains and durations. As with MD simulations, the HDM comes with the price of very high computational cost.

Therefore, in retrospective, sampling of long trajectories of channel gating with the HDM, as presented in chapter 5 may be seen as an overeager approach for the topic. That said, while involving a high computational effort, the results of chapter 5 still showed the first proof of concept of the central hypothesis of this thesis: unbinding of $\mathrm{IP}_{3}$ from $\mathrm{IP}_{3}$ Rs introduces a second level of dynamics, which enables waves as a second release pattern besides puffs already on a single cluster level. Even more important, the results from the HDM allowed the development of the coarse grained model (CGM), as the different spatial and temporal scales of $\mathrm{Ca}^{2+}$ release are accessible in an almost arbitrary precision.
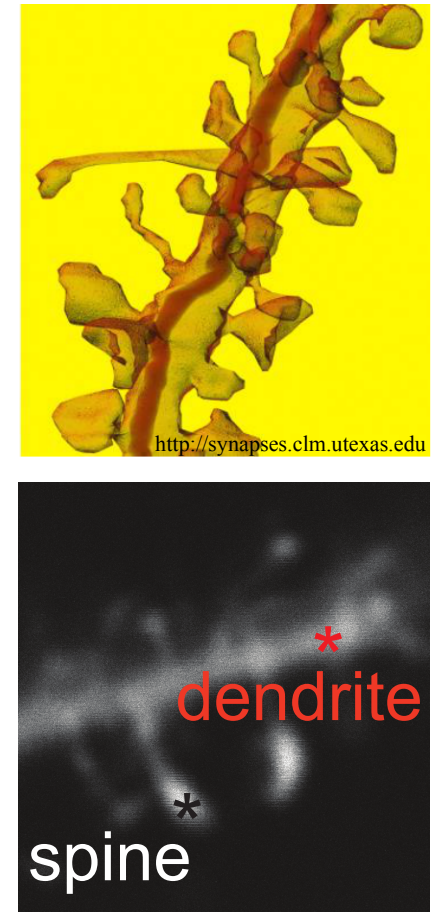

Fig. 7.1: Spiny dendrites. Top: Reconstruction from electron microscopy. Bottom: Light microscope image with parallel fluorescence measurements from $[\mathrm{Joh}+15]$. 
Besides providing the basis for the phenomenological description of calcium in the CGM the high level of detail can be very useful for answering some other questions. E.g. in $[\mathrm{Joh}+15]$, the interference of dye buffers with the natural dynamics of $\mathrm{Ca}^{2+}$ and $\mathrm{IP}_{3} \mathrm{Rs}$ in dendritic spines was quantified with a very similar deterministic model as presented in chapter 5. Dendritic spines as depicted in fig. 7.1 often resemble the junction between dendrite and synapse and serve as excellent subject for applications of the HDM: their spatial extent is sufficiently small, but the exact $\mathrm{Ca}^{2+}$ distribution within the spine is often of major interest. Further, the FEM and the software toolkit that was used throughout chapter 5 generally allows for complex boundaries of the simulation domain, which means that using a realistic spine geometry obtained from experimental data by electron microscopy is generally possible.

As a downside, future simulations of a larger domain with many clusters will required certain approximations to be introduced into the algorithm. One of them could be the integration of subdomains containing single clusters with their own internal adaptive time steps. This approach ultimately means that discretization of space and time do not separate anymore and hence requires careful error estimations and poses a very challenging implementation.

The knowledge about time, space, and concentration scales, which was obtained in chapter 5, was used to develop a coarse grained model in chapter 6 . This new model provides an intuitive understanding of the interplay between the random gating of $\mathrm{IP}_{3} \mathrm{R}$ and the multi-scale calcium concentrations. Because of its reduced number of parameters and phenomenological nature it is much easier to understand and predict than the HDM. Further, its numerical simplicity allows to quickly explore different parameter regimes for much larger system sizes and time scales, because the required CPU time is reduced by a couple of magnitudes. It is capable of sampling trajectories of a couple of hours involving the gating of a total of $1024 \mathrm{IP}_{3} \mathrm{Rs}$ leading to millions of individual channel transitions. Thus, similar to the variety of simplified neuron models in neuroscience, the CGM can serve as a signpost to understand the complex dynamics of large systems with emergent complex behavior.

Such long trajectories are required for sampling statistically significant results for event properties and synchronization measurements. In the case of the grid of coupled clusters of $\mathrm{IP}_{3} \mathrm{Rs}$ this work was able to demonstrate, that many of the previously reported features of $\mathrm{IP}_{3} \mathrm{R}$ dynamics, can also be reproduced with a discrete state representation of the $\mathrm{IP}_{3} \mathrm{R}$ channels. For example, a single cluster may exhibit most regular release via puffs for a certain optimal number of $\mathrm{IP}_{3} \mathrm{R}$ [SJ02]. One year later, it was demonstrated that a grid of coupled clusters synchronizes best for certain inter cluster distances [SJ03]. A very similar coherence resonance effect was demonstrated in 
chapter 6 , where the coherence between the coupled clusters peaks at a certain $\mathrm{IP}_{3}$ concentration.

The majority of the publications dealing with models of $\mathrm{IP}_{3} \mathrm{Rs}$ use Langevin equations to describe a joint state of multiple channels (e.g. all channels of one cluster) via dynamic continuous variables. This pooled treatment of discrete states essentially resembles a coarse grained approach of the channel dynamics. Hence, it is especially problematic for low occupation numbers, where the discrete nature of fluctuations starts to play a role. There exist different strategies to avoid such problems, e.g. by introducing a Langevin description for subunits [HRS15], or by introducing an artificial term that deals with the occupation numbers close to zero [Rud14b]. In contrast to that, this work showed that a properly implemented model can easily deal with up to 1000 individual channels, involving a total of 8000 different state variables. Thereby it circumvents the problems arising from continuous gating variables. Further, chapter 6 demonstrated that the used discrete description is equally capable of producing resonance effects which are known from models based on Langevin equations.

Together, both chapters 5 and 6 suggest that the $\mathrm{IP}_{3}$ binding dynamics of the $\mathrm{IP}_{3} \mathrm{R}$ cannot be neglected or assumed to be in equilibrium during $\mathrm{Ca}^{2+}$ release events in general. Instead, the binding and unbinding of $\mathrm{IP}_{3}$ can explain multiple features of experimentally observed $\mathrm{Ca}^{2+}$ release patterns:

- It allows modulation of release between puffs and waves.

- It provides an alternative release termination mechanism kicking in when inhibition fails to terminate prolonged release events at elevated $\mathrm{Ca}^{2+}$ levels.

- It can explain passive $\mathrm{IP}_{3}$ oscillations which follow global $\mathrm{Ca}^{2+}$ oscillations.

- It is a possible explanation for the variability of puff amplitudes.

Apparently all the findings above heavily rely on the used DYK gating schema for the $\mathrm{IP}_{3} \mathrm{R}$. While the parameters which were used for the gating rates originate from experimental measurements, not all of them are directly accessible. In fact, many of the rates are still subject to approximations and assumptions. This weak spot hopefully can be filled with the help of new computational methods such as Markov state models (MSMs) of molecular kinetics. These relatively new techniques provide the mathematical toolset to consistently extract gating rates between the most relevant coformational changes of molecules [Pri+11; Koh+14]. They are based on extensive molecular dynamics simulations on the atomistic scale, where the complete configuration of proteins is sampled over a couple of microseconds. The very high dimensional state space of a protein's configuration then is reduced to a much smaller number of relevant states and 
the transition rates between these states are extracted. Recent advances in this area of research have allowed to simulate large membrane proteins in a cloud based manner and may soon provide results for the $\mathrm{IP}_{3} \mathrm{R}$ and its exact gating properties [Shu+15].

Circling back to the introduction of this work, there are different requirement on a model to be considered useful. First, the level of detail has to be properly distributed over different aspects of system of interest. In the HDM the balance slightly tended towards the modeling of $\mathrm{Ca}^{2+}$ diffusion and buffering. In contrast to that, the CGM absorbed the uncertainties in parameters of $\mathrm{Ca}^{2+}$ diffusion and buffering in its phenomenological description, tilting the scale towards the channel gating level of detail. As claimed in the above paragraphs, both of these approaches make sense, since they serve a different purpose. It is a choice of toolset that has to be made for the question at hand, and it made sense to apply different tools for different questions. 


\section{Bibliography}

[AMS92] Nancy L Allbritton, Tobias Meyer, and Lubert Stryer. "Range of messenger action of calcium ion and inositol 1, 4, 5-trisphosphate". In: Science 258 (1992), pp. 1812-1812.

doi: $10.1126 /$ science.1465619.

[Ani+07] Vadim S Anishchenko, Vladimir Astakhov, Alexander Neiman, Tatjana Vadivasova, and Lutz Schimansky-Geier. Nonlinear dynamics of chaotic and stochastic systems: tutorial and modern developments. Springer Science \& Business Media, 2007.

[Atr+93] Alireza Atri, Jeff Amundson, David Clapham, and James Sneyd. "A singlepool model for intracellular calcium oscillations and waves in the Xenopus laevis oocyte". In: Biophysical Journal 65.4 (1993), pp. 1727-1739. doi: 10.1016/S0006-3495(93)81191-3.

[Bar+00] M. Bär, M. Falcke, L. Tsimring, and H. Levine. "Discrete stochastic modeling of calcium channel dynamics". In: Phys.Rev.Lett. 84 (2000), pp. 56645667.

doi: 10.1103/PhysRevLett.84.5664.

[Bas+08a] Peter Bastian, Markus Blatt, Andreas Dedner, Christian Engwer, Robert Klöfkorn, Ralf Kornhuber, Markus Ohlberger, and Oliver Sander. "A generic grid interface for parallel and adaptive scientific computing. Part II: Implementation and tests in DUNE". In: Computing 82.2-3 (2008), pp. 121-138. doi: 10.1007/s00607-008-0004-9.

[Bas+08b] Peter Bastian, Markus Blatt, Andreas Dedner, Christian Engwer, Robert Klöfkorn, Markus Ohlberger, and Oliver Sander. "A generic grid interface for parallel and adaptive scientific computing. Part I: abstract framework". In: Computing 82.2-3 (2008), pp. 103-119. doi: $10.1007 / \mathrm{s} 00607-008-0003-\mathrm{x}$. 
[BB07] Markus Blatt and Peter Bastian. The iterative solver template library. Springer, 2007. doi: 10.1007/978-3-540-75755-982.

[BB08] Markus Blatt and Peter Bastian. "On the generic parallelisation of iterative solvers for the finite element method". In: International Journal of Computational Science and Engineering 4.1 (2008), pp. 56-69. doi: 10.1504/IJCSE.2008.021112.

[BD87] G.E.P. Box and N.R. Draper. Empirical model-building and response surfaces. Wiley series in probability and mathematical statistics: Applied probability and statistics. Wiley, 1987. ISBN: 9780471810339. URL: http s://books . google.de/books?id=Q02dDRuf JEAC.

[Ber14] Michael J. Berridge. "Module 10: Neuronal Signalling”. In: Cell Signalling Biology 6 (2014). ISSN: 1749-7787. doi: $10.1042 /$ csb0001010.

[BF07] K Bentele and M Falcke. "Quasi-steady approximation for ion channel currents". In: Biophysical journal 93.8 (2007), pp. 2597-2608. doi: 10.1529/biophysj.107.104299.

[BHM10] Peter Bastian, Felix Heimann, and Sven Marnach. "Generic implementation of finite element methods in the distributed and unified numerics environment (DUNE)". In: Kybernetika 46.2 (2010), pp. 294-315.

[BLB00] M.J. Berridge, P. Lipp, and M.D. Bootman. "The versatility and universality of calcium signalling". In: Nature Rev. Mol. Cell Biol. 1 (2000), pp. 1122 .

doi: $10.1038 / 35036035$.

[Bos14] N. Bostrom. Superintelligence: Paths, Dangers, Strategies. Oxford University Press, 2014. ISBN: 9780199678112. URL: https://books.google.de /books?id=7\_H8AwAAQBAJ.

[Bow+15] Michael Bowling, Neil Burch, Michael Johanson, and Oskari Tammelin. "Heads-up limit hold'em poker is solved". In: Science 347.6218 (2015), pp. 145-149. ISSN: 0036-8075.

doi: $10.1126 /$ science.1259433.

$[$ Bru+10] Luciana Bruno, Guillermo Solovey, Alejandra C Ventura, Sheila Dargan, and Silvina Ponce Dawson. "Quantifying calcium fluxes underlying calcium puffs in Xenopus laevis oocytes". In: Cell calcium 47.3 (2010), pp. 273- 
286.

doi: 10.1016/j.ceca.2009.12.012.

[Cal+98] N. Callamaras, J.S. Marchant, X.-P. Sun, and I. Parker. "Activation and co-ordination of $\mathrm{InsP}_{3}$-mediated elementary $\mathrm{Ca}^{2+}$ events during global $\mathrm{Ca}^{2+}$ signals in Xenopus oocytes". In: Journal of Physiology 509.1 (1998), pp. $81-91$.

doi: 10.1111/j.1469-7793.1998.081bo.x.

[Can+13] MB Cannell, CHT Kong, MS Imtiaz, and DR Laver. "Control of sarcoplasmic reticulum $\mathrm{Ca} 2+$ release by stochastic RyR gating within a $3 \mathrm{D}$ model of the cardiac dyad and importance of induction decay for CICR termination”. In: Biophysical journal 104.10 (2013), pp. 2149-2159.

doi: 10.1016/j.bpj.2013.03.058.

$[\mathrm{Cao}+13]$ Pengxing Cao, Graham Donovan, Martin Falcke, and James Sneyd. "A Stochastic Model of Calcium Puffs Based on Single-Channel Data". In: Biophysical journal 105.5 (2013), pp. 1133-1142. doi: 10.1016/j.bpj.2013.07.034.

[CDS16] Matthieu Chavent, Anna L Duncan, and Mark SP Sansom. "Molecular dynamics simulations of membrane proteins and their interactions: from nanoscale to mesoscale". In: Current Opinion in Structural Biology 40 (2016). Carbohydrate-protein interactions and glycosylation, Biophysical and molecular biological methods, pp. 8-16. ISSN: 0959-440X.

doi: 10.1016/j.sbi.2016.06.007.

[CK91] R. E. Challis and R. I. Kitney. "Biomedical signal processing (in four parts)". In: Medical and Biological Engineering and Computing 29.3 (1991), pp. 225-241. ISSN: 1741-0444.

doi: 10.1007/BF02446704.

[CKN73] G Carter, C Knapp, and A Nuttall. "Estimation of the magnitude-squared coherence function via overlapped fast Fourier transform processing". In: IEEE transactions on audio and electroacoustics 21.4 (1973), pp. 337-344. doi: 10.1109/TAU.1973.1162496.

[CM13] Joseph D Challenger and Alan J McKane. "Synchronization of stochastic oscillators in biochemical systems". In: Physical Review E 88.1 (2013), p. 012107.

doi: 10.1103/PhysRevE.88.012107. 
[CP00] Nick Callamaras and Ian Parker. "Phasic characteristic of elementary $\mathrm{Ca}^{2+}$ release sites underlies quantal responses to $\mathrm{IP}_{3}$ ". In: The EMBO Journal 19.14 (2000), pp. 3608-3617.

doi: $10.1093 / \mathrm{emboj} / 19.14 .3608$.

[CT03] S Coombes and Y Timofeeva. "Sparks and waves in a stochastic fire-diffusefire model of $\mathrm{Ca}^{2+}$ release". In: Physical Review E 68.2 (2003), p. 021915. doi: 10.1103/PhysRevE.68.021915.

[DAC08] G. Dupont, A. Abou-Lovergne, and L. Combettes. "Stochastic Aspects of Oscillatory $\mathrm{Ca}^{2+}$ Dynamics in Hepatocytes". In: Biophysical Journal 95.5 (2008), pp. 2193-2202.

[DK92] G. DeYoung and J. Keizer. "A single-pool inositol 1,4,5-trisphosphatereceptor-based model for agonist-stimulated oscillations in $\mathrm{Ca}^{2+}$ concentration". In: Proc. Natl. Acad. Sci USA 89 (1992), pp. 9895-9899. doi: $10.1073 /$ pnas.89.20.9895.

[DM11] Luis Diambra and Jonathan S Marchant. "Inositol (1, 4, 5)-trisphosphate receptor microarchitecture shapes $\mathrm{Ca}^{2+}$ puff kinetics". In: Biophysical journal 100.4 (2011), pp. 822-831.

doi: 10.1016/j.bpj.2011.01.003.

[DP03] S.L. Dargan and I. Parker. "Buffer kinetics shape the spatiotemporal patterns of $\mathrm{IP}_{3}$-evoked $\mathrm{Ca}^{2+}$ signals". In: J.Physiol. 553.3 (2003), pp. 775-788. doi: 10.1113/jphysiol.2003.054247.

[DP13] George D Dickinson and Ian Parker. "Factors determining the recruitment of inositol trisphosphate receptor channels during calcium puffs". In: Biophysical journal 105.11 (2013), pp. 2474-2484.

doi: $10.1016 /$ j.bpj.2013.10.028.

[DSP12] George D Dickinson, Divya Swaminathan, and Ian Parker. "The probability of triggering calcium puffs is linearly related to the number of inositol trisphosphate receptors in a cluster". In: Biophysical journal 102.8 (2012), pp. $1826-1836$.

doi: 10.1016/j.bpj.2012.03.029.

[Dup+11] Geneviève Dupont, Laurent Combettes, Gary S Bird, and James W Putney. "Calcium oscillations". In: Cold Spring Harbor perspectives in biology 3.3 (2011), a004226.

doi: 10.1101/cshperspect.a004226. 
[DW12] Peter Deuflhard and Martin Weiser. Adaptive numerical solution of PDEs. Walter de Gruyter, 2012. ISBN: 978-3-11-028311-2.

[Fal03] M. Falcke. "On the role of stochastic channel behavior in intracellular $\mathrm{Ca}^{2+}$ dynamics". In: Biophys.J. 84.1 (2003), pp. 42-56.

doi: 10.1016/S0006-3495(03)74831-0.

[Fal04] M. Falcke. "Reading the patterns in living cells - the Physics of $\mathrm{Ca}^{2+}$ signaling". In: Advances in Physics 53.3 (2004), pp. 255-440.

doi: 10.1080/00018730410001703159.

[Fav+96] Cécile J Favre, Jacques Schrenzel, Jean Jacquet, Daniel P Lew, and KarlHeinz Krause. "Highly supralinear feedback inhibition of $\mathrm{Ca}^{2+}$ uptake by the $\mathrm{Ca}^{2+}$ load of intracellular stores". In: Journal of Biological Chemistry 271.25 (1996), pp. 14925-14930.

doi: $10.1074 /$ jbc.271.25.14925.

[Fit61] Richard FitzHugh. "Impulses and physiological states in theoretical models of nerve membrane". In: Biophysical journal 1.6 (1961), pp. 445-466. doi: 10.1016/S0006-3495(61)86902-6.

[FOB00] M. Falcke, M. Or-Guil, and M. Bär. "Dispersion gap and localized spiral waves in a model for intracellular $\mathrm{Ca}^{2+}$ dynamics". In: Phys.Rev.Lett. 84.20 (2000), pp. 4753-4756.

doi: 10.1103/PhysRevLett.84.4753.

[For15] Michael D Forrest. "Simulation of alcohol action upon a detailed Purkinje neuron model and a simpler surrogate model that runs; 400 times faster". In: BMC neuroscience 16.1 (2015), p. 27.

doi: 10.1186/s12868-015-0162-6.

[Fos+07] J.K. Foskett, C. White, K.H. Cheung, and D.O.D. Mak. "Inositol Trisphosphate Receptor $\mathrm{Ca}^{2+}$ Release Channels". In: Physiol. Rev. 87.2 (2007), p. 593.

doi: 10.1152/physrev.00035.2006.

[FRE13] Mark B Flegg, Sten Rüdiger, and Radek Erban. "Diffusive spatio-temporal noise in a first-passage time model for intracellular calcium release". In: The Journal of chemical physics 138.15 (2013), p. 154103. doi: 10.1063/1.4796417.

[FTL00] M. Falcke, L. Tsimring, and H. Levine. "Stochastic spreading of intracellular $\mathrm{Ca}^{2+}$ release". In: Phys.Rev.E 62 (2000), pp. 2636-2643.

doi: 10.1103/PhysRevE.62.2636. 
[Gas+14] Lawrence D Gaspers, Paula J Bartlett, Antonio Politi, Paul Burnett, Walson Metzger, Jane Johnston, Suresh K Joseph, Thomas Höfer, and Andrew P Thomas. "Hormone-induced calcium oscillations depend on crosscoupling with inositol 1, 4, 5-trisphosphate oscillations". In: Cell reports 9.4 (2014), pp. 1209-1218.

doi: $10.1016 /$ j.celrep.2014.10.033.

[Gil76] D.T. Gillespie. "A general method for numerically simulating the stochastic time evolution of coupled chemical reactions". In: Journal of computational physics 22.4 (1976), pp. 403-434. ISSN: 0021-9991.

doi: 10.1016/0021-9991(76)90041-3.

[Gi191] D.T. Gillespie. Markov Processes: An Introduction for Physical Scientists. Elsevier Science, 1991. ISBN: 9780080918372. URL: https://books.googl e.de/books?id=4HgC7a8V4iEC.

[GS08] Jeffrey R Groff and Gregory D Smith. "Calcium-dependent inactivation and the dynamics of calcium puffs and sparks". In: Journal of theoretical biology 253.3 (2008), pp. 483-499.

doi: $10.1016 /$ j.jtbi.2008.03.026.

[HH52] Alan L Hodgkin and Andrew F Huxley. "A quantitative description of membrane current and its application to conduction and excitation in nerve". In: The Journal of physiology 117.4 (1952), p. 500.

[Hon02] Josef Honorkamp. Statistical physics: an advanced approach with applications. 2002.

[HRS15] Yandong Huang, Sten Rüdiger, and Jianwei Shuai. "Accurate Langevin approaches to simulate Markovian channel dynamics". In: Physical Biology 12.6 (2015), p. 061001.

doi: 10.1088/1478-3975/12/6/061001.

[IKL88] Giuseppe Inesi, Mark Kurzmack, and David Lewis. "Kinetic and equilibrium characterization of an energy-transducing enzyme and its partial reactions". In: Methods in enzymology 157 (1988), pp. 154-190.

doi: 10.1016/0076-6879(88)57074-X.

[Ion+07] Lucian Ionescu, Carl White, King-Ho Cheung, Jianwei Shuai, Ian Parker, John E Pearson, J Kevin Foskett, and Don-On Daniel Mak. "Mode switching is the major mechanism of ligand regulation of InsP3 receptor calcium release channels". In: The Journal of general physiology 130.6 (2007), 
pp. $631-645$.

doi: $10.1085 /$ jgp.200709859.

[Jia+02] Qiu-Xing Jiang, Edwin C Thrower, David W Chester, Barbara E Ehrlich, and Fred J Sigworth. "Three-dimensional structure of the type 1 inositol 1, 4, 5-trisphosphate receptor at $24 \AA$ resolution". In: The EMBO journal 21.14 (2002), pp. 3575-3581.

doi: $10.1093 /$ emboj/cdf380.

$[$ Joh+15] Friedrich W Johenning, Anne-Kathrin Theis, Ulrike Pannasch, Martin Rückl, Sten Rüdiger, and Dietmar Schmitz. "Ryanodine receptor activation induces long-term plasticity of spine calcium dynamics". In: PLoS Biol 13.6 (2015), e1002181.

doi: 10.1371/journal.pbio.1002181.

[JRW89] Suresh K Joseph, HL Rice, and John R Williamson. "The effect of external calcium and $\mathrm{pH}$ on inositol trisphosphate-mediated calcium release from cerebellum microsomal fractions". In: Biochemical Journal 258.1 (1989), pp. 261-265.

doi: $10.1042 / \mathrm{bj} 2580261$.

[KD94] J. Keizer and G.W. DeYoung. "Simplification of a realistic model of $\mathrm{IP}_{3}$ induced $\mathrm{Ca}^{2+}$ oscillations". In: J.theor.Biol. 166 (1994), pp. 431-442. doi: 10.1006/jtbi.1994.1038.

$[$ Koh+14] Kai J Kohlhoff, Diwakar Shukla, Morgan Lawrenz, Gregory R Bowman, David E Konerding, Dan Belov, Russ B Altman, and Vijay S Pande. "Cloud-based simulations on Google Exacycle reveal ligand modulation of GPCR activation pathways". In: Nature chemistry 6.1 (2014), pp. 1521.

doi: $10.1038 /$ nchem.1821.

[KS10] James Keener and James Sneyd. Mathematical Physiology: I: Cellular Physiology. Springer Science \& Business Media, 2010. ISBN: 978-0-38775846-6. doi: 10.1007/978-0-387-75847-3.

[Kur+17] F Kurz, CH Ziener, M Rückl, A Hahn, VJ Sturm, K Zhang, LR Buschle, M Bendszus, S Heiland, HP Schlemmer, WR Bauer, and T Kampf. "The influence of spatial patterns of capillary networks on transverse relaxation". In: Magnetic Resonance Imaging 40 (2017), pp. $31-47$.

doi: 10.1016/j.mri.2017.03.012. 
[Le+11] Quoc V. Le, Rajat Monga, Matthieu Devin, Greg Corrado, Kai Chen, Marc'Aurelio Ranzato, Jeffrey Dean, and Andrew Y. Ng. "Building highlevel features using large scale unsupervised learning". In: CoRR abs/1112.6209 (2011). URL: http://arxiv.org/abs/1112.6209.

[LJE91] Bezprozvanny Llya, Watras James, and Ehrlich Barbara E. "Bell-shaped calcium-response curves of Ins(1,4,5)P3- and calcium-gated channels from endoplasmic reticulum of cerebellum". In: Nature 351.6329 (1991), pp. 751754 .

doi: $10.1038 / 351751 \mathrm{a} 0$.

[LR94] Y.-X. Li and J. Rinzel. "Equations for $\mathrm{InsP}_{3}$ receptor-mediated $\left[\mathrm{Ca}^{2+}\right]_{i}$ oscillations derived from a detailed kinetic model: A Hodgkin-Huxley like formalism". In: J.theor.Biol. 166 (1994), pp. 461-473. doi: $10.1006 /$ jtbi.1994.1041.

[Lyt+92] J. Lytton, M. Westlin, S.E. Burk, G.E. Shull, and D.H. MacLennan. "Functional comparisons between isoforms of the sarcoplasmic or endoplasmic reticulum family of calcium pumps". In: J.Biol.Chem. 267.20 (1992), pp. 1448314489. URL: http://europepmc.org/abstract/MED/1385815.

$[$ Mak+07] Don-On Daniel Mak, John E Pearson, King Pan Campion Loong, Suman Datta, Marisabel Fernández-Mongil, and J Kevin Foskett. "Rapid ligandregulated gating kinetics of single inositol 1,4,5-trisphosphate receptor $\mathrm{Ca}^{2+}$ release channels". In: EMBO reports 8.11 (2007), pp. 1044-1051. ISSN: 1469-221X. doi: $10.1038 /$ sj.embor.7401087.

[Mar87] S Lawrence Marple Jr. "Digital spectral analysis with applications". In: Englewood Cliffs, NJ, Prentice-Hall, Inc., 1987, 512 p. 1 (1987).

[MCP99] Jonathan Marchant, Nick Callamaras, and Ian Parker. "Initiation of $\mathrm{IP}_{3^{-}}$ mediated $\mathrm{Ca}^{2+}$ waves in Xenopus oocytes". In: The EMBO Journal 18.19 (1999), pp. 5285-5299. doi: $10.1093 /$ emboj/18.19.5285.

[MF97] Don-On Daniel Mak and J Kevin Foskett. "Single-channel kinetics, inactivation, and spatial distribution of inositol trisphosphate $\left(\mathrm{IP}_{3}\right)$ receptors in Xenopus oocyte nucleus". In: The Journal of general physiology 109.5 (1997), pp. 571-587. doi: 10.1085/jgp.109.5.571. 
[MMF98] Don-On Daniel Mak, Sean McBride, and J Kevin Foskett. "Inositol 1, 4, 5-tris-phosphate activation of inositol tris-phosphate receptor $\mathrm{Ca}^{2+}$ channel by ligand tuning of $\mathrm{Ca}^{2+}$ inhibition". In: Proceedings of the National Academy of Sciences 95.26 (1998), pp. 15821-15825.

doi: $10.1073 /$ pnas.95.26.15821.

[MP01] J.S. Marchant and I. Parker. "Role of elementary $\mathrm{Ca}^{2+}$ puffs in generating repetitive $\mathrm{Ca}^{2+}$ oscillations". In: The EMBO Journal 20.1 \& 2 (2001), pp. $65-76$.

doi: 10.1093/emboj/20.1.65.

[MP98] Jacopo Meldolesi and Tullio Pozzan. "The endoplasmic reticulum $\mathrm{Ca}^{2+}$ store: a view from the lumen". In: Trends in biochemical sciences 23.1 (1998), pp. 10-14.

doi: 10.1016/S0968-0004(97)01143-2.

[Nag+07] Chamakuri Nagaiah et al. "Adaptive numerical simulation of reaction: diffusion systems". PhD thesis. Citeseer, 2007.

[NAY62] Jinichi Nagumo, Suguru Arimoto, and Shuji Yoshizawa. "An active pulse transmission line simulating nerve axon". In: Proceedings of the IRE 50.10 (1962), pp. 2061-2070.

doi: 10.1109/JRPROC.1962.288235.

[Neh86] E. Neher. "Concentration profiles of intracellular calcium in the presence of a diffusible chelator". In: Calcium electrogenesis and neuronal functioning (1986), pp. 80-96.

[Niv+13] Michael Nivala, Christopher Y Ko, Melissa Nivala, James N Weiss, and Zhilin Qu. "The emergence of subcellular pacemaker sites for calcium waves and oscillations". In: The Journal of physiology 591.21 (2013), pp. 53055320 .

doi: 10.1113/jphysiol.2013.259960.

$[$ Par +08$] \quad$ R Madelaine Paredes, Julie C Etzler, Lora Talley Watts, Wei Zheng, and James D Lechleiter. "Chemical calcium indicators". In: Methods 46.3 (2008), pp. 143-151.

doi: 10.1016/j.ymeth.2008.09.025.

[Pat96] Raj Kumar Pathria. Statistical mechanics. 2nd ed. Elsevier, 1996. 
[Pri+11] Jan-Hendrik Prinz, Hao Wu, Marco Sarich, Bettina Keller, Martin Senne, Martin Held, John D. Chodera, Christof Schütte, and Frank Noé. "Markov models of molecular kinetics: Generation and validation". In: The Journal of Chemical Physics 134.17 (2011), p. 174105.

doi: $10.1063 / 1.3565032$.

[Pri81] Maurice Bertram Priestley. "Spectral analysis and time series". In: (1981).

[PRK01] Arkady Pikovsky, Michael Rosenblum, and Jürgen Kurths. A universal concept in nonlinear sciences. Cambridge University Press, 2001.

[QKG02] R Quian Quiroga, T Kreuz, and P Grassberger. "Event synchronization: a simple and fast method to measure synchronicity and time delay patterns". In: Physical review E 66.4 (2002), p. 041904.

doi: 10.1103/PhysRevE.66.041904.

[Red14] Junuthula Narasimha Reddy. An Introduction to Nonlinear Finite Element Analysis: with applications to heat transfer, fluid mechanics, and solid mechanics. OUP Oxford, 2014.

[RJS12] S. Rüdiger, P. Jung, and J.W. Shuai. "Termination of $\mathrm{Ca}^{2+}$ Release for Clustered $\mathrm{IP}_{3} \mathrm{R}$ Channels". In: PLoS Computational Biology 8.5 (2012), e1002485.

doi: 10.1371/journal.pcbi.1002485.

[RLS11] Hena R Ramay, Ona Z Liu, and Eric A Sobie. "Recovery of cardiac calcium release is controlled by sarcoplasmic reticulum refilling and ryanodine receptor sensitivity". In: Cardiovascular research (2011), cvr143.

doi: $10.1093 / \mathrm{cvr} / \mathrm{cvr} 143$.

[Roc87] Michel Roche. "Rosenbrock methods for Differential Algebraic Equations". English. In: Numerische Mathematik 52.1 (1987), pp. 45-63. ISSN: 0029$599 X$.

doi: 10.1007/BF01401021.

[RR16] Martin Rückl and Sten Rüdiger. "Calcium waves in a grid of clustered channels with synchronous $\mathrm{IP}_{3}$ binding and unbinding". In: The European Physical Journal E 39.11 (2016), p. 108.

doi: 10.1140/epje/i2016-16108-4.

[RS09] S. Rüdiger and L. Schimansky-Geier. "Dynamics of excitable elements with time-delayed coupling". In: J. Theor. Biol. 259 (2009), pp. 96-100.

doi: 10.1016/j.jtbi.2009.01.030. 
[RSS10] S. Rüdiger, J.W. Shuai, and I.M. Sokolov. "Law of Mass Action, Detailed Balance, and the Modeling of Calcium Puffs". In: Phys. Rev. Lett. 105 (2010), p. 048103.

doi: 10.1103/PhysRevLett.105.048103.

[Ruc+15] Martin Rückl, Ian Parker, Jonathan S Marchant, Chamakuri Nagaiah, Friedrich W Johenning, and Sten Rüdiger. "Modulation of elementary calcium release mediates a transition from puffs to waves in an $\mathrm{IP}_{3} \mathrm{R}$ cluster model”. In: PLoS Comput Biol 11.1 (2015), e1003965.

doi: 10.1371/journal.pcbi.1003965.

[Rud+07] S. Rüdiger, J.W. Shuai, W. Huisinga, Ch. Nagaiah, G. Warnecke, I. Parker, and M. Falcke. "Hybrid Stochastic and Deterministic Simulations of Calcium Blips". In: Biophys. J. 93 (2007), pp. 1847-1857.

doi: 10.1529/biophysj.106.099879.

$[$ Rud +10$] \quad$ S. Rüdiger, Ch. Nagaiah, G. Warnecke, and J.W. Shuai. "Calcium Domains around Single and Clustered $\mathrm{IP}_{3}$ Receptors and Their Modulation by Buffers". In: Biophys. J. 99.1 (2010), pp. 3-12.

doi: 10.1016/j.bpj.2010.02.059.

[Rud14a] S Rüdiger. "Excitability in a stochastic differential equation model for calcium puffs". In: Physical Review E 89.6 (2014), p. 062717.

doi: 10.1103/PhysRevE.89.062717.

[Rud14b] Sten Rüdiger. "Stochastic Models of Intracellular Calcium Signals". In: Phys. Rep., 534.2 (2014), pp. 39-87.

doi: $10.1016 /$ j.physrep.2013.09.002.

[Rue+17] Martin Rueckl, Stephen C Lenzi, Laura Moreno-Velasquez, Daniel Parthier, Dietmar Schmitz, Sten Ruediger, and Friedrich W Johenning. "SamuROI, a python-based software tool for visualization and analysis of dynamic time series imaging at multiple spatial scales". In: Frontiers in neuroinformatics 11 (2017), p. 44.

doi: 10.3389/fninf.2017.00044.

[Sch+15] Christian Schmeltzer, Alexandre Hiroaki Kihara, Igor Michailovitsch Sokolov, and Sten Rüdiger. "Degree correlations optimize neuronal network sensitivity to sub-threshold stimuli". In: PloS one 10.6 (2015), e0121794. doi: 10.1371/journal.pone.0121794. 
[Sch89] HJ Schatzmann. "The calcium pump of the surface membrane and of the sarcoplasmic reticulum". In: Annual review of physiology 51.1 (1989), pp. $473-485$.

doi: 10.1146/annurev.ph.51.030189.002353.

[Shu+09] JW Shuai, DP Yang, JE Pearson, and S. Rüdiger. "An investigation of models of the IPR channel in Xenopus oocyte". In: Chaos 19 (2009), p. 037105 .

doi: $10.1063 / 1.3156402$.

[Shu+15] Diwakar Shukla, Carlos X Hernández, Jeffrey K Weber, and Vijay S Pande. "Markov state models provide insights into dynamic modulation of protein function". In: Accounts of chemical research 48.2 (2015), pp. 414-422. doi: 10.1021/ar5002999.

[Sil+16] David Silver, Aja Huang, Chris J Maddison, Arthur Guez, Laurent Sifre, George Van Den Driessche, Julian Schrittwieser, Ioannis Antonoglou, Veda Panneershelvam, Marc Lanctot, et al. "Mastering the game of Go with deep neural networks and tree search". In: Nature 529.7587 (2016), pp. 484-489. doi: 10.1038/nature16961.

[SJ02] J.W. Shuai and P. Jung. "Optimal intracellular calcium signalling". In: Phys.Rev.Lett. 88.6 (2002), doi: 10.1103/PhysRevLett.88.068102.

[SJ03] J.W. Shuai and P. Jung. "Optimal ion channel clustering for intracellular calcium signaling". In: Proc.Nat.Acad.Sci.USA 100.2 (2003), pp. 506-510. doi: $10.1073 /$ pnas. 0236032100 .

$[$ Sku+08] A. Skupin, H. Kettenmann, U. Winkler, M. Wartenberg, H. Sauer, S.C. Tovey, C.W. Taylor, and M. Falcke. "How Does Intracellular $\mathrm{Ca}^{2+}$ Oscillate: By Chance or by the Clock?" In: Biophysical Journal 94.6 (2008), p. 2404.

doi: 10.1529/biophysj.107.119495.

[SL10] Irina I. Serysheva and Steven J. Ludtke. "Chapter 8 - 3D Structure of IP3 Receptor". In: Structure and Function of Calcium Release Channels. Ed. by Irina I. Serysheva. Vol. 66. Current Topics in Membranes. Academic Press, 2010, pp. 171-189.

doi: 10.1016/S1063-5823(10)66008-5. 
[Smi+01] G.D. Smith, L. Dai, R.M. Miura, and A. Sherman. "Asymptotic analysis of buffered calcium diffusion near a point source". In: SIAM J.Appl.Math. 61.5 (2001), pp. 1816-1838.

doi: $10.1137 /$ S0036139900368996.

[Smi07] Julius O Smith. Mathematics of the discrete Fourier transform (DFT): with audio applicaitons. Julius Smith, 2007.

$[$ Sol +08$] \quad$ G. Solovey, D. Fraiman, B. Pando, and S. Ponce Dawson. "Simplified model of cytosolic $\mathrm{Ca}^{2+}$ dynamics in the presence of one or several clusters of $\mathrm{Ca}^{2+}$-release channels". In: Physical Review E 78.4 (2008), p. 41915. ISSN: $1550-2376$.

doi: 10.1103/PhysRevE.78.041915.

[SP05] Jianwei Shuai and Ian Parker. "Optical single-channel recording by imaging $\mathrm{Ca}^{2+}$ flux through individual ion channels: theoretical considerations and limits to resolution". In: Cell calcium 37.4 (2005), pp. 283-299.

[SP09] Ian F Smith and Ian Parker. "Imaging the quantal substructure of single $\mathrm{IP}_{3} \mathrm{R}$ channel activity during $\mathrm{Ca}^{2+}$ puffs in intact mammalian cells". In: Proceedings of the National Academy of Sciences 106.15 (2009), pp. 64046409.

doi: 10.1073/pnas.0810799106.

[SRP06] Jianwei Shuai, Heather J. Rose, and Ian Parker. "The Number and Spatial Distribution of $\mathrm{IP}_{3}$ Receptors Underlying Calcium Puffs in Xenopus Oocytes". In: Biophysical Journal 91.11 (2006), pp. 4033-4044.

doi: 10.1529/biophysj.106.088880.

[SUJ09] D. Swaminathan, G. Ullah, and P. Jung. "A simple sequential-binding model for calcium puffs". In: Chaos 19 (2009), p. 037109.

doi: $10.1063 / 1.3152227$.

[Sun+98] X.-P. Sun, N. Callamaras, J.S. Marchant, and I. Parker. "A continuum of $\mathrm{InsP}_{3}$-mediated elementary $\mathrm{Ca}^{2+}$ signalling events in Xenopus oocytes". In: Journal of Physiology 509.1 (1998), pp. 67-80.

doi: 10.1111/j.1469-7793.1998.067bo.x.

[Swi+99] Stéphane Swillens, Geneviève Dupont, Laurent Combettes, and Philippe Champeil. "From calcium blips to calcium puffs: theoretical analysis of the requirements for interchannel communication". In: Proceedings of the National Academy of Sciences 96.24 (1999), pp. 13750-13755.

doi: 10.1073/pnas.96.24.13750. 
[Tak+16] Silvia Honda Takada, Juliane Midori Ikebara, Erica de Sousa, Débora Sterzeck Cardoso, Rodrigo Ribeiro Resende, Henning Ulrich, Martin Rückl, Sten Rüdiger, and Alexandre Hiroaki Kihara. "Determining the Roles of Inositol Trisphosphate Receptors in Neurodegeneration: Interdisciplinary Perspectives on a Complex Topic". In: Molecular Neurobiology (2016), pp. $1-15$. doi: 10.1007/s12035-016-0205-8.

[Tan+09] Akihiko Tanimura, Takao Morita, Akihiro Nezu, Akiko Shitara, Noboru Hashimoto, and Yosuke Tojyo. "Use of fluorescence resonance energy transferbased biosensors for the quantitative analysis of inositol 1, 4, 5-trisphosphate dynamics in calcium oscillations". In: Journal of Biological Chemistry 284.13 (2009), pp. 8910-8917. doi: $10.1074 /$ jbc.M805865200.

[Tau+09] Taufiq-Ur-Rahman, Alexander Skupin, Martin Falcke, and Colin W Taylor. "Clustering of $\mathrm{InsP}_{3}$ receptors by $\mathrm{InsP}_{3}$ retunes their regulation by $\mathrm{InsP}_{3}$ and $\mathrm{Ca}^{2+}$ ". In: Nature 458.7238 (2009), pp. 655-659. doi: $10.1038 /$ nature07763.

[Ter+13] Mark Terasaki, Tom Shemesh, Narayanan Kasthuri, Robin W Klemm, Richard Schalek, Kenneth J Hayworth, Arthur R Hand, Maya Yankova, Greg Huber, Jeff W Lichtman, et al. "Stacked endoplasmic reticulum sheets are connected by helicoidal membrane motifs". In: Cell 154.2 (2013), pp. 285-296. doi: $10.1016 /$ j.cell.2013.06.031.

[TF04] R. Thul and M. Falcke. "Release currents of $\mathrm{IP}_{3}$ receptor channel clusters and concentration profiles". In: Biophys.J. 86 (2004), pp. 2660-2673. doi: 10.1016/S0006-3495(04)74322-2.

$[\mathrm{Thu}+12] \quad$ Kevin Thurley, Alexander Skupin, Rüdiger Thul, and Martin Falcke. "Fundamental properties of $\mathrm{Ca}^{2+}$ signals". In: Biochimica et Biophysica Acta (BBA)-General Subjects 1820.8 (2012), pp. 1185-1194. doi: $10.1016 /$ j.bbagen.2011.10.007.

$[\mathrm{Thu}+14] \quad$ Kevin Thurley, Stephen C Tovey, Gregor Moenke, Victoria L Prince, Abha Meena, Andrew P Thomas, Alexander Skupin, Colin W Taylor, and Martin Falcke. "Reliable Encoding of Stimulus Intensities Within Random Sequences of Intracellular $\mathrm{Ca}^{2+}$ Spikes". In: Science signaling 7.331 (2014), ra59-ra59.

doi: 10.1126/scisignal.2005237. 
[TTF09] Ruediger Thul, K Thurley, and Martin Falcke. "Towards a predictive model of $\mathrm{Ca}^{2+}$ puffs". In: Chaos 19 (2009), p. 037108.

doi: 10.1063/1.3183809.

[Ull+12] G. Ullah, I. Parker, D.O.D. Mak, and J.E. Pearson. "Multi-scale datadriven modeling and observation of calcium puffs". In: Cell Calcium 52 (2012), p. 152.

doi: 10.1016/j.ceca.2012.04.018.

[Van92] Nicolaas Godfried Van Kampen. Stochastic processes in physics and chemistry. Vol. 1. Elsevier, 1992.

[VFM10] Horia Vais, J Kevin Foskett, and Don-On Daniel Mak. "Unitary $\mathrm{Ca}^{2+}$ current through recombinant type $3 \mathrm{InsP}_{3}$ receptor channels under physiological ionic conditions". In: The Journal of general physiology 136.6 (2010), pp. $687-700$.

doi: $10.1085 /$ jgp.201010513.

[Wel67] Peter Welch. "The use of fast Fourier transform for the estimation of power spectra: a method based on time averaging over short, modified periodograms". In: IEEE Transactions on audio and electroacoustics (1967), pp. $70-73$.

doi: 10.1109/TAU.1967.1161901.

[WH91] G Wanner and E Hairer. Solving ordinary differential equations II. Vol. 1. Springer-Verlag, Berlin, 1991.

[WK94] J. Wagner and J. Keizer. "Effects of rapid buffers on $\mathrm{Ca}^{2+}$ diffusion and $\mathrm{Ca}^{2+}$ oscillations". In: Biophys.J. 67 (1994), pp. 447-456. doi: 10.1016/S0006-3495(94)80500-4.

[Zie+12] CH Ziener, M Rückl, T Kampf, WR Bauer, and HP Schlemmer. "Mathieu functions for purely imaginary parameters". In: Journal of Computational and Applied Mathematics 236.17 (2012), pp. 4513-4524.

doi: 10.1016/j.cam.2012.04.023.

[ZKH01] Changsong Zhou, Jürgen Kurths, and Bambi Hu. "Array-enhanced coherence resonance: nontrivial effects of heterogeneity and spatial independence of noise". In: Physical review letters 87.9 (2001), p. 098101.

doi: 10.1103/PhysRevLett.87.098101. 


\section{Selbstständigkeitserklärung}

Hiermit versichere ich, dass ich die vorliegende Arbeit selbstständig verfasst, und keine anderen als die angegebenen Quellen und Hilfsmittel verwendet habe.

Berlin, 03.05.2017

Martin Rückl 



\section{Publications}

- CH Ziener, M Rückl, T Kampf, WR Bauer, and HP Schlemmer. "Mathieu functions for purely imaginary parameters". In: Journal of Computational and Applied Mathematics 236.17 (2012), pp. 4513-4524.

doi: $10.1016 /$ j.cam.2012.04.023

- Martin Rückl, Ian Parker, Jonathan S Marchant, Chamakuri Nagaiah, Friedrich W Johenning, and Sten Rüdiger. "Modulation of elementary calcium release mediates a transition from puffs to waves in an $\mathrm{IP}_{3} \mathrm{R}$ cluster model". In: PLoS Comput Biol 11.1 (2015), e1003965.

doi: 10.1371/journal.pcbi.1003965

- Friedrich W Johenning, Anne-Kathrin Theis, Ulrike Pannasch, Martin Rückl, Sten Rüdiger, and Dietmar Schmitz. "Ryanodine receptor activation induces longterm plasticity of spine calcium dynamics". In: PLoS Biol 13.6 (2015), e1002181. doi: 10.1371/journal.pbio.1002181

- Martin Rückl and Sten Rüdiger. "Calcium waves in a grid of clustered channels with synchronous $\mathrm{IP}_{3}$ binding and unbinding". In: The European Physical Journal E 39.11 (2016), p. 108.

doi: 10.1140/epje/i2016-16108-4

- Silvia Honda Takada, Juliane Midori Ikebara, Erica de Sousa, Débora Sterzeck Cardoso, Rodrigo Ribeiro Resende, Henning Ulrich, Martin Rückl, Sten Rüdiger, and Alexandre Hiroaki Kihara. "Determining the Roles of Inositol Trisphosphate Receptors in Neurodegeneration: Interdisciplinary Perspectives on a Complex Topic". In: Molecular Neurobiology (2016), pp. 1-15.

doi: $10.1007 / \mathrm{s} 12035-016-0205-8$

- F Kurz, CH Ziener, M Rückl, A Hahn, VJ Sturm, K Zhang, LR Buschle, M Bendszus, S Heiland, HP Schlemmer, WR Bauer, and T Kampf. "The influence of spatial patterns of capillary networks on transverse relaxation". In: Magnetic Resonance Imaging 40 (2017), pp. $31-47$.

doi: 10.1016/j.mri.2017.03.012

- Martin Rueckl, Stephen C Lenzi, Laura Moreno-Velasquez, Daniel Parthier, Dietmar Schmitz, Sten Ruediger, and Friedrich W Johenning. "SamuROI, a pythonbased software tool for visualization and analysis of dynamic time series imaging at multiple spatial scales". In: Frontiers in neuroinformatics 11 (2017), p. 44. doi: $10.3389 /$ fninf.2017.00044 